Predictive Validity of the Dynamic Risk Assessment for Offender Re-Entry Among Intimate Partner Violence Offenders

by

Bronwen Perley-Robertson

A thesis submitted to the Faculty of Graduate and Postdoctoral Affairs in partial fulfillment of the requirements for the degree of

\title{
Master of Arts
}

in

Psychology

Carleton University

Ottawa, Ontario

(C) 2018

Bronwen Perley-Robertson 


\begin{abstract}
Intimate partner violence (IPV) is among the most common acts of violence against women worldwide, making it a major global threat to women's health and safety. The assessment and management of IPV offenders are therefore vital tasks in criminal justice systems. The current study examined whether the DRAOR, a case management tool, predicted repeat partner abuse among 112 male IPV offenders in Iowa, U.S. While the DRAOR did not predict IPV recidivism in this sample, it appears to be useful for informing case management decisions among partnerviolent men. Risk factors that emerged as important treatment targets were poor attachment with others, substance abuse, anger/hostility, opportunity/access to victims, problematic interpersonal relationships, and overall acute risk. Further research is needed to improve the utility of the DRAOR for predicting IPV recidivism, but this study tentatively supports the use of the DRAOR for supervising IPV offenders until an IPV-specific case management tool is developed.
\end{abstract}




\section{Acknowledgements}

I want to first thank my supervisor, Dr. Ralph Serin, for offering me this invaluable opportunity. I have appreciated your ongoing support and guidance, as well as your generosity in gifting me budding research projects! My gratitude also goes to my committee members, Drs. Shelley Brown and Adelle Forth, for their constructive input into my thesis, and to Dr. Diana Majury, my internal examiner, for sharing her thoughts and perspective at my defence. It is also with the utmost gratitude that I acknowledge Drs. L. Maaike Helmus and Andrew Smith for offering their statistical expertise. Maaike, I am especially appreciative of your endless guidance over the years-you have influenced my work in many ways and I am forever grateful. A special thank you to Nick Chadwick as well for preparing and assisting with this dataset and for helping focus my analytic plan in the beginning stages.

There are many other people to whom I owe thanks, but foremost to my family, whose encouragement has been integral to my success. To my mother, Diane, and my brother, Evan, thank you for your understanding, support, and love. I am certain that I would not be here, at the end of my master's degree, if it were not for you. My gratitude also goes to Julia Burpee, my oldest friend, for always graciously agreeing to edit my work. You have such a beautiful way with words and I'm so lucky to have your influence in my writing. I want to thank my wonderful circle of friends as well for giving me some balance in my life; I have enjoyed our countless cottage weekends, game nights, potluck dinners, and coffee outings over the years. I'm incredibly fortunate to know you all!

Finally, to my father, who has always encouraged my academic endeavours and inspired my senses of compassion and determination. I feel very honoured to be involved in this line of work and I know my father would've been proud. 


\section{Table of Contents}

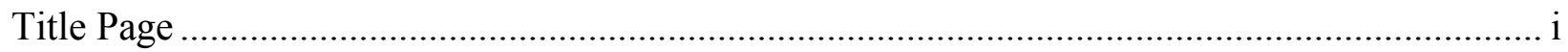

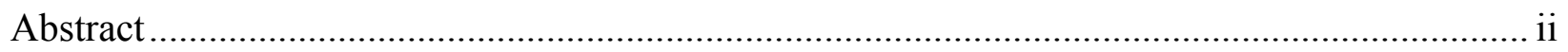



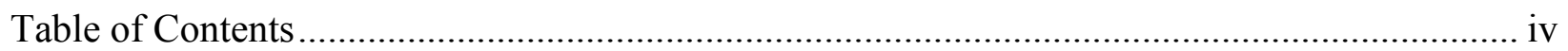

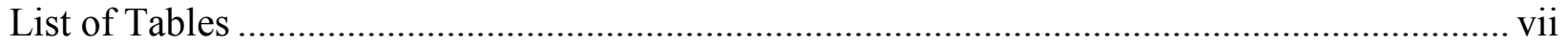

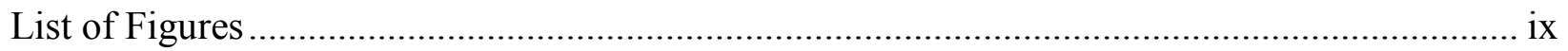

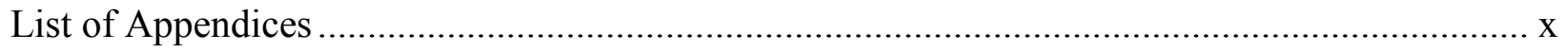

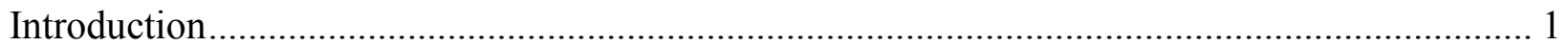

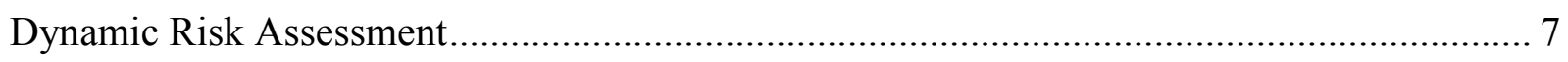

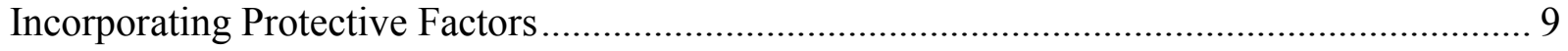

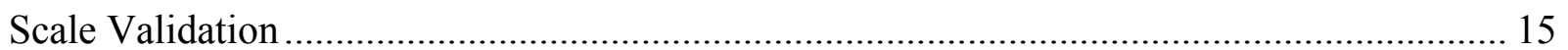

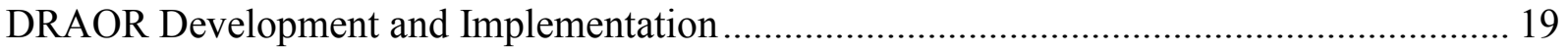

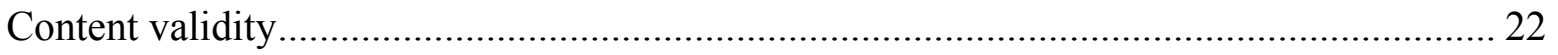



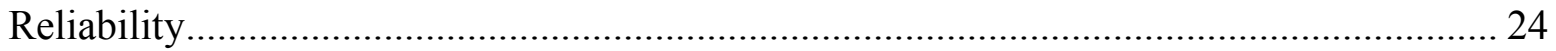

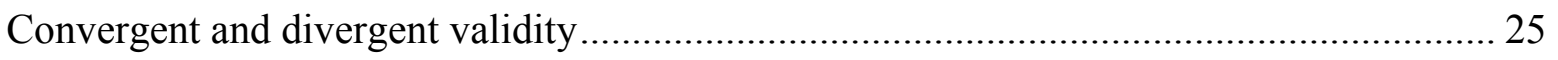



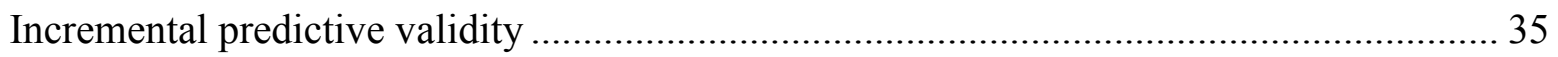



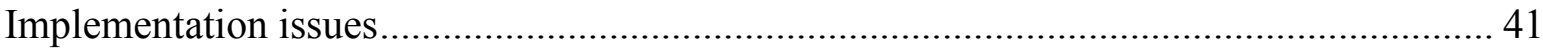

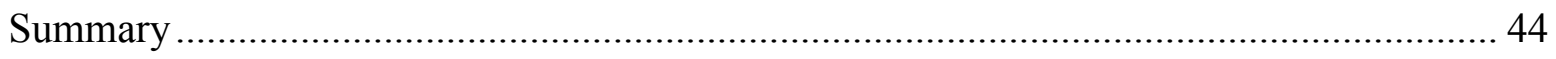

DRAOR With Intimate Partner Violence Offenders .......................................................... 45 


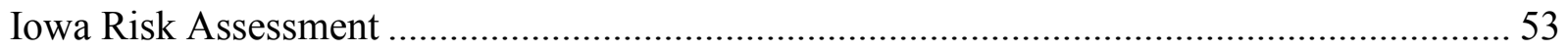

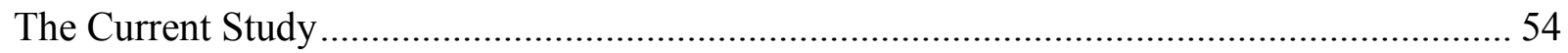

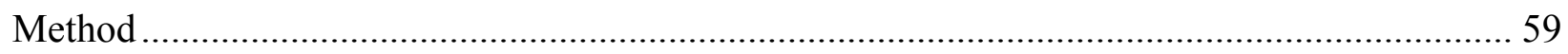

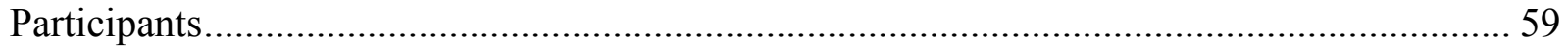

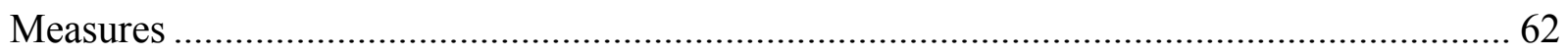

DRAOR

IRA

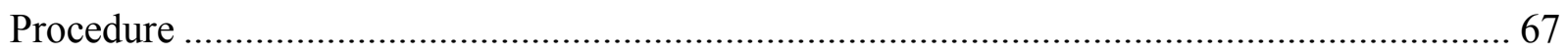

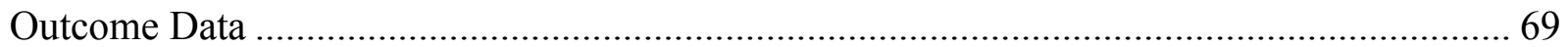

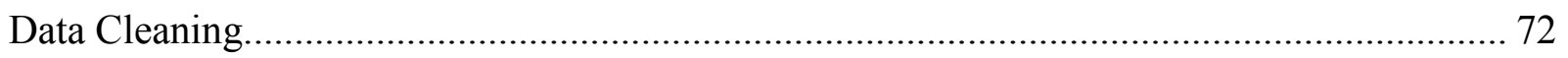

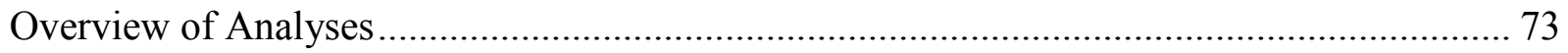

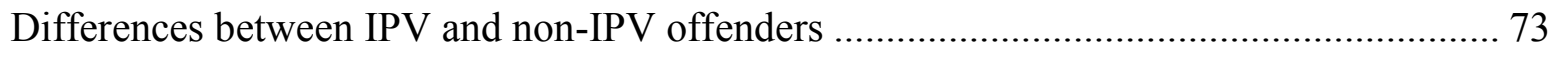

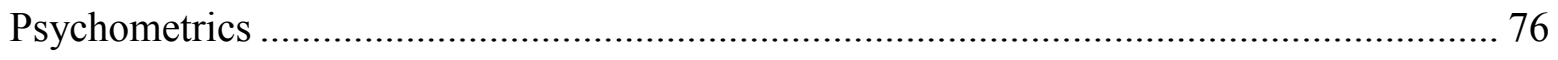

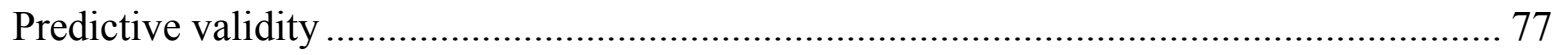

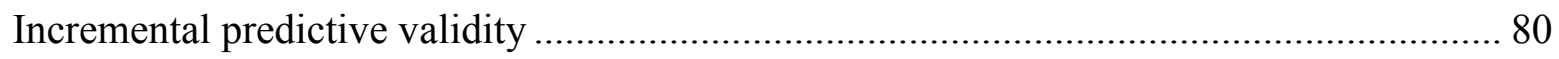

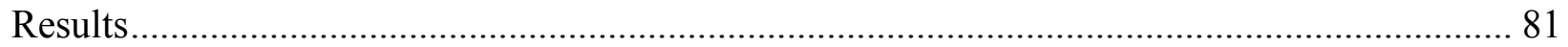

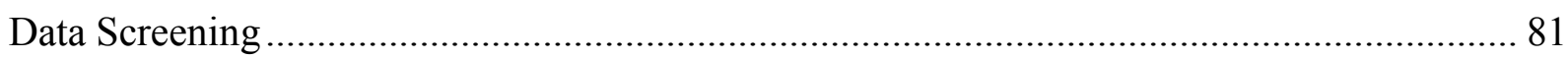

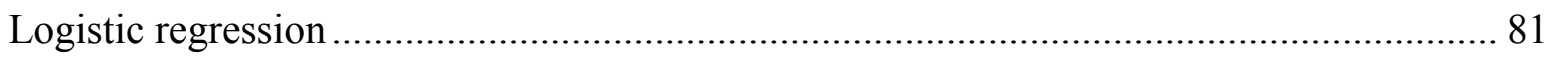

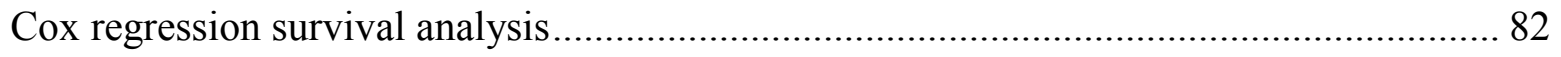

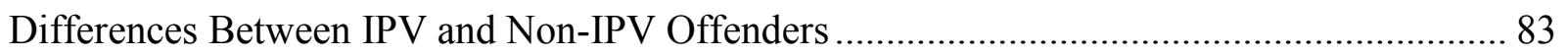

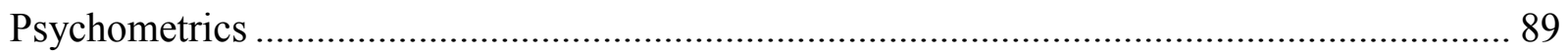



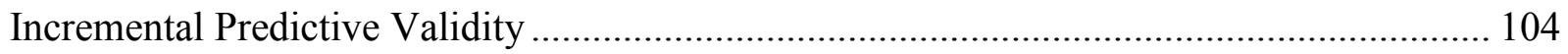






Differences Between IPV and Non-IPV Offenders ...................................................... 106

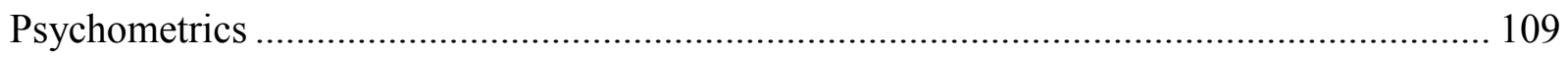

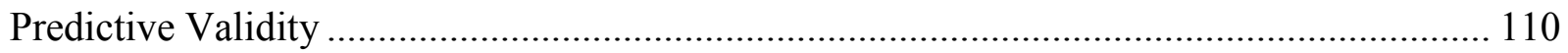

Incremental Predictive Validity ......................................................................... 114

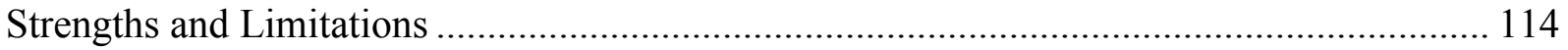

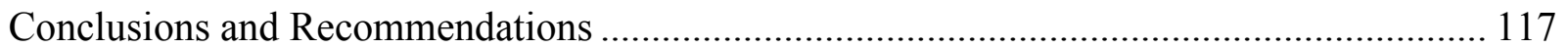

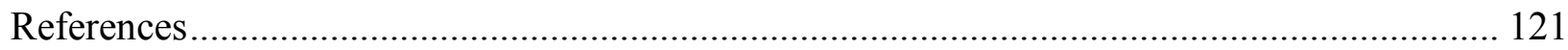




\section{List of Tables}

Table 1. DRAOR Predictive Validity Results (Point-Biserial Correlations and AUCs) Reported in

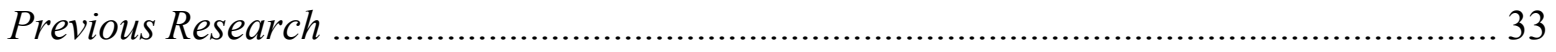

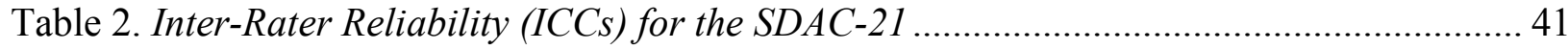

Table 3. Demographic Information for IPV and Non-IPV Offenders ..................................... 61

Table 4. Number of History, Index, and Recidivism IPV Offenders and Incidents .................... 69

Table 5. Follow-up Time in Months (Ms and SDs) and Outcome Base Rates for Overall Sample,

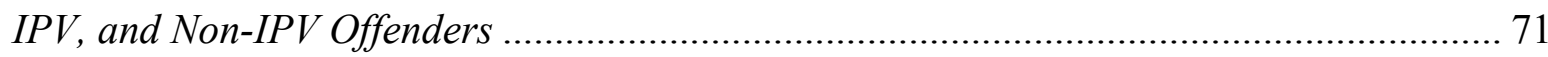

Table 6. DRAOR Descriptives (Ms and SDs) for IPV and Non-IPV Offenders ........................ 85

Table 7. Differences in DRAOR Scores (AUCs and Cohen's ds) Between IPV and Non-IPV

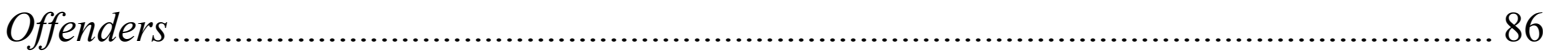

Table 8. Outcome Base Rates Across Risk Level for IPV and Non-IPV Offenders .................... 88

Table 9. IPV and Non-IPV Offenders in Each DRAOR Risk Level (ns and \%)......................... 89

Table 10. DRAOR Item-Total Correlations and Cronbach's Alphas if Item Deleted for IPV

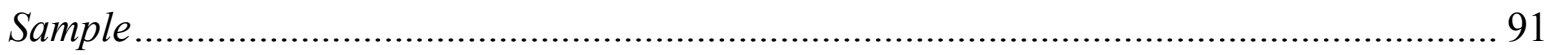

Table 11. Correlations Between Risk and Protective Measures for IPV Sample ....................... 92

Table 12. Predictive Validity of the DRAOR and IRA (AUCs) Across Outcomes for IPV Sample

Table 13. Predictive Validity of DRAOR Items (AUCs) for IPV Recidivism ............................. 95

Table 14. Logistic Regression Analyses for DRAOR Total Scores Predicting Community

Outcomes Among Overall Sample, IPV, and Non-IPV Offenders .................................. 98

Table 15. Likelihood of Community Outcomes for DRAOR Risk Level Cut-offs Among IPV and Non-IPV Offenders 103 
Table 16. Univariate Cox Regression Survival Analyses Predicting Time to Outcomes Among

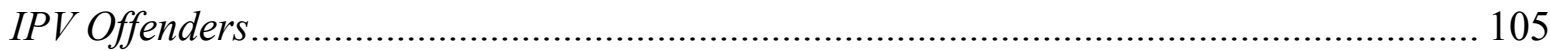




\section{List of Figures}

Figure 1. Ecological model for male-perpetrated intimate partner violence.

Figure 2. Probability of general recidivism across DRAOR Total scores and risk level for the overall sample

Figure 3. Probability of IPV recidivism across DRAOR Total scores and risk level for the overall sample 100

Figure 4. Probability of violent recidivism across DRAOR Total scores and risk level for the overall sample 101

Figure 5. Probability of technical violations across DRAOR Total scores and risk level for the overall sample 102 


\section{List of Appendices}

Appendix A. Dynamic Risk Assessment for Offender Re-Entry (DRAOR) ......................... 156

Appendix B. Description of DRAOR Items ................................................................. 159

Appendix C. Structured Dynamic Assessment Case-Management-21 (SDAC-21)................. 163

Appendix D. Iowa Risk Assessment (IRA) ................................................................. 166 
Predictive Validity the Dynamic Risk Assessment for Offender Re-Entry Among Intimate Partner Violence Offenders

Intimate partner violence (IPV) is among the most common acts of violence against women worldwide, making it a major global threat to women's health and safety. Roughly 30 to $35 \%$ of women worldwide are victimized by an intimate partner in their lifetime (Devries et al., 2013; World Health Organization [WHO], 2017). In the United States (U.S.), over 1 in 3 (37.3\%) women reported experiencing physical violence, contact sexual violence, and/or stalking by an intimate partner in their lifetime (Smith et al., 2017). Further, IPV results in more treatment for injuries each year in the U.S. than any other cause of injury to women (Anderson, Stockman, Sabri, Campbell, \& Campbell, 2015). Research from the National Center for Injury Prevention and Control estimates that physical IPV results in 1.8 million injuries per year, with $28.8 \%$ of those requiring medical attention (Gerberding, Binder, Hammond, \& Arias, 2003). The prevalence of IPV in the U.S. is seen in Canada as well; namely, it is the leading type of violence experienced by women (Burczycka \& Conroy, 2017a, 2017b; Burczycka, Conroy, \& Savage, 2018). Recent surveys also found that 8 in 10 victims of police-reported IPV cases in Canada were women (Burczycka \& Conroy, 2017b; Burczycka et al., 2018).

IPV is linked to a multitude of negative physical health effects including, but not limited to, Human Immunodeficiency Virus (HIV; Kouyoumdjian et al., 2013; Silverman, Decker, Saggurti, Balaiah, \& Raj, 2008), traumatic brain injury (McFadgion, 2014; Valera \& Kucyi, 2017), gastrointestinal issues (Campbell, 2002; Coker, Smith, Bethea, King, \& McKeown, 2000), stress and behaviours that lead to cardiovascular disease (Scott-Storey, Wuest, \& FordGilboe, 2009), as well as neurological issues (Dutton et al., 2006). IPV during pregnancy is related to miscarriage, late entry into prenatal care, stillbirth, premature labour and birth, fetal 
injury, and low-birth-weight or small-for-gestational-age infants (WHO \& Pan American Health Organization [PAHO] 2012a). Beyond physical injuries, victims report many negative emotional and psychological impacts of IPV, including fear, concern for one's safety, post-traumatic stress disorder, depression, anxiety, phobias, eating and sleep disorders, physical inactivity, and poor self-esteem (Smith et al., 2017; WHO \& PAHO, 2012a). These problems, not surprisingly, are exacerbated for women who experience severe IPV (WHO \& PAHO, 2012a).

Physical IPV is a precursor to intimate partner femicide (the murder of women by intimate partners) in 67 to $80 \%$ of cases (Campbell et al., 2003). Worldwide, intimate partner femicide is the largest homicide risk for women. It is estimated that, globally, 35 to $37 \%$ of female homicide victims are murdered by an intimate partner - a number that is likely on the conservative side (Stöckl et al., 2013; WHO \& PAHO, 2012b). Effective IPV risk assessments and interventions that are empirically-based may, therefore, reduce intimate partner femicide.

The public health costs of physical and sexual IPV against women was estimated at $\$ 5.8$ billion in 1995 in the U.S. (\$4.2 billion for physical assault, $\$ 893$ million for murders, $\$ 342$ million for stalking, and $\$ 320$ million for rapes), which translated to roughly $\$ 8.3$ billion in 2003 (Max, Rice, Finkelstein, Bardwell, \& Leadbetter, 2004), a number that is exponentially higher today due to inflation and a growing population. Comparable costs have been estimated in Canada (Zhang, Hoddenbagh, McDonald, \& Scrim, 2012), the United Kingdom (U.K.; Walby, 2004), Australia (KPMG, 2016), and New Zealand (Kahui \& Snively, 2014). Taken together, these statistics highlight the continuing need for effective IPV assessment, prevention, and intervention.

The effectiveness of any IPV intervention effort depends, in part, on whether it targets factors that are relevant to the risk of IPV. A good understanding of risk factors is therefore 
important for effective social and criminal justice responses. Evidently, our understanding of IPV risk factors is rooted in the development of different IPV theories, beginning with nested ecological models, first generated in the 1980s (Dutton, 1985). These models allow for a multidimensional explanation of IPV, involving numerous individual, relationship, community, and social factors that influence IPV risk over time. Dahlberg and Krug (2002) provide a description of an ecological model for the perpetration of violence, which was used by the WHO and the London School of Hygiene and Tropical Medicine in their publication aimed at preventing intimate partner and sexual violence against women (Butchart, Garcia-Moreno, \& Mikton, 2010). This model is summarized below (see also Figure 1).

At the individual level, violence perpetration risk is influenced by biological and personal variables, such as demographics (e.g., low income, education, young age), childhood maltreatment/exposure to violence, personality/mood disorders (e.g., antisocial personality disorder, depression), substance abuse, fear of rejection, and anger/hostility. Relationship variables involve a person's closest social circle (i.e., peers, intimate partners, family members), which can shape their behaviour and experiences. The offender having relationships with antisocial others or living with his victim, can increase the likelihood of violence, for example. At the community level, characteristics of the settings in which social relationships are embedded (e.g., schools, workplaces, and neighbourhoods) can contribute to the likelihood of violence perpetration. Weak community sanctions, poverty, and frequent changes in housing, for example, may lead to increased violence. Finally, the societal level includes broad, macro-level factors that influence violence through the creation of inequalities or tensions between groups of people, such as traditional gender norms and social norms supportive of violence. 


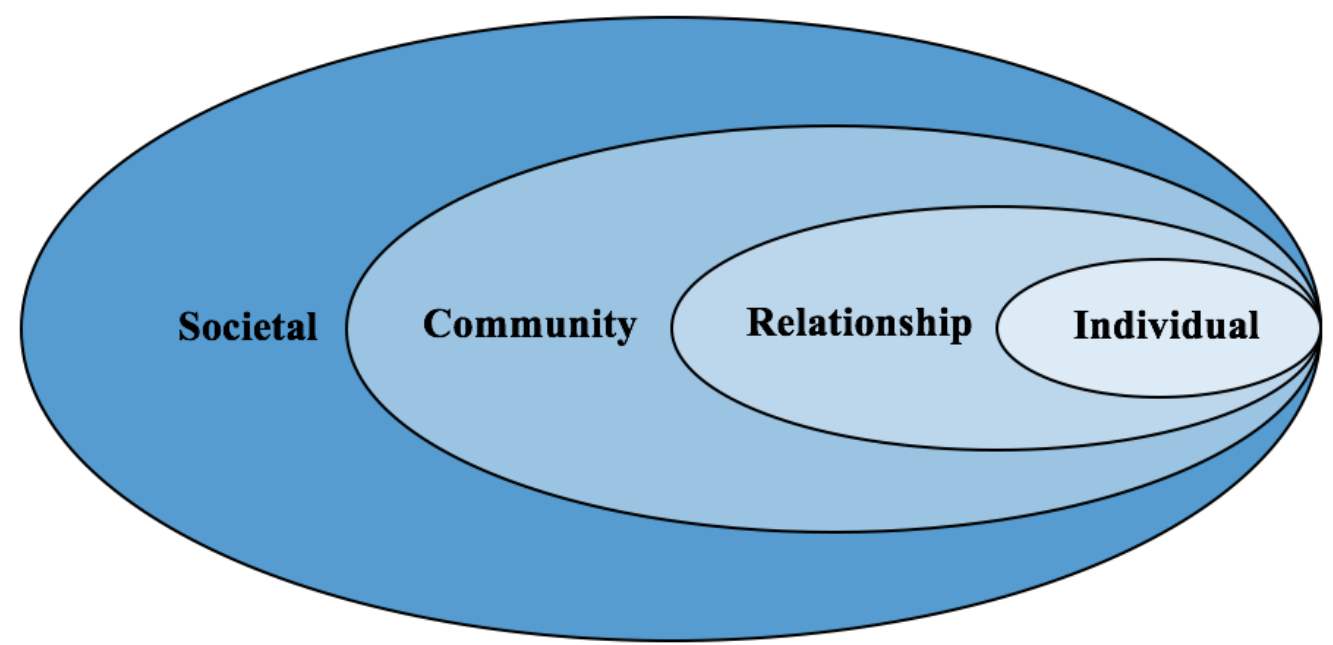

Figure 1. Ecological model for male-perpetrated intimate partner violence (Dahlberg \& Krug, 2002).

During the 1990s, social learning theories dominated the literature due to a heightened concern for children's exposure to violence and the risk of intergenerational transmission of violence (e.g., Hilton, 1992; Jaffe, Wolfe \& Wilson, 1990). Specifically, intergenerational transmission theory contends that adults who witnessed or experienced family violence during childhood are more likely to imitate this aggression in intimate relationships (Sellers, Cochran, \& Branch, 2005). Male peer support theory is another type of social learning theory, except it emphasizes the transmission of patriarchal group values from peers, rather than the transmission of family violence across generations (Sellers et al., 2005). Under this theory, males are said to seek support from members of their male-only social groups (e.g., fraternities, athletic teams, gangs) when they feel their authority has been challenged by their female partners (Sellers et al., 2005). Patriarchal values held by these male-only social groups often justify, legitimize, and encourage physical aggression towards females (Sellers et al., 2005).

Research on predictors of IPV recidivism expanded in the early 2000 s and structured methods of assessing risk for IPV recidivism among at-risk individuals were validated (i.e., 
methods that moved away from the reliance on clinical judgment, including actuarial and structured professional judgment approaches; Hilton et al., 2004; Kropp \& Hart, 2000; Williams \& Houghton, 2004). These tools are now widely used across many jurisdictions and the search for improved prediction of IPV recidivism has been central to the IPV research literature in the current decade. The three most extensively researched IPV-specific risk scales include the Ontario Domestic Assault Risk Assessment (ODARA; Hilton, Harris, \& Rice, 2010), the Domestic Violence Screening Instrument (DVSI; Williams \& Houghton, 2004), and the Spousal Assault Risk Assessment guide (SARA; Kropp, Hart, Webster, \& Eaves, 1994, 1995, 1998). The ODARA and DVSI are actuarial risk measures designed to aid in the prediction of repeat domestic assault. Predicting the likelihood that an offender will engage in further criminal activity involves both the selection of variables on which to focus, as well as appropriately combining these variables to form recidivism estimates (Hilton, Harris, \& Rice, 2006). The actuarial method of risk assessment involves selecting the strongest set of recidivism predictors identified in methodologically sound follow-up studies and then adding only those items that improve the predictive accuracy of this optimum set (Hilton et al., 2006). Notably, it is this method of item selection that distinguishes actuarial measures from other types of measures (Hilton et al., 2006), and it is the process that was undertaken by the developers of the ODARA and DVSI.

The second defining feature of the actuarial method concerns how items are combined to allow for the estimation of recidivism risk. Specifically, risk factors are combined using item weights that are based on empirical relationships between risk factors and recidivism (Hilton et al., 2006). Stronger predictors of recidivism are weighted more heavily than weaker predictors, or if the differential weighting of predictors does not impact the accuracy of risk estimates, items 
can be weighted equally (Hilton et al., 2006). Item scores are then summed to reach a final score, which is interpreted based on established norms (the proportion of cases falling above or below a certain score) and experience tables (the proportion of offenders at a certain score who recidivated; Hilton et al., 2006, pp. 402).

Actuarial tools consist mainly of static risk factors- - historical variables that are not amenable to change, such as number of previous convictions or age at first offence-and provide a statistical estimate of recidivism risk. These measures have been shown to predict general, violent, and sexual recidivism more accurately than clinical judgment approaches (Campbell, French, \& Gendreau, 2009; Hanson \& Morton-Bourgon, 2009; Hilton et al., 2006), which require correctional decision makers to form unstructured clinical opinions on the probability of offending behaviour based on intuition and professional experience (Bonta, 1996). One limitation of actuarial methods, however, is that static risk factors do not capture changes in risk (Douglas \& Skeem, 2005). As such, actuarial tools cannot inform risk management beyond using baseline measures of risk to determine the frequency of contact between supervision officers and offenders.

The SARA is a structured professional judgment (SPJ) instrument designed to aid in the prediction of IPV recidivism. The SPJ model is an approach that requires correctional decision makers to evaluate risk by considering empirically-based risk factors (Borum, 1996). An important distinction between SPJ and actuarial instruments is that the SPJ approach does not provide probabilities for reoffending. Instead, qualitative evaluations of risk are provided, such as potential types of reoffending, when reoffending is most likely to occur, and how to prevent recidivism (Hart \& Cooke, 2013). Meta-analytic results show support for the utility of the SPJ model, suggesting it is comparable to actuarial methods (Guy, 2010). Clinical judgments based 
on structured evaluations of offenders can therefore provide meaningful contributions to the assessment of risk.

Given the diverse range of serious consequences associated with IPV, it is important to study factors related to this type of violence. The objective of the current study is to examine the validity of the Dynamic Risk Assessment for Offender Re-Entry (DRAOR) — a measure used statewide in Iowa for assessing risk in all community-supervised offenders - among partnerviolent men. The following discussion gives an overview of static and dynamic risk factors, protective factors, and evidence for their use in risk assessment. Elements pertinent to the scale validation process are summarized next, followed by a review of the development and implementation of the DRAOR. Research on the relation of DRAOR items to IPV recidivism is then presented, warranting the current study's inquiry into the usefulness of the tool with IPV offenders. This section concludes with a series of research questions and hypotheses aimed at validating the DRAOR for use with partner-violent men.

\section{Dynamic Risk Assessment}

Instruments that evaluate solely static risk factors do not provide the opportunity to document changes in risk beyond those due to aging or new criminal behaviour. While static risk factors are valid predictors of recidivism (Helmus, Thornton, Hanson, \& Babchishin, 2012; McCoy \& Miller, 2013; Philipse, Koeter, van der Staak, \& van den Brink, 2006), research demonstrating the utility of offender change information in predicting recidivism is becoming more prominent (Brown, St. Amand, \& Zamble, 2009; Hanson, Harris, Scott, \& Helmus, 2007;

Lewis, Olver, \& Wong, 2012; Van Voorhis, Wright, Salisbury, \& Bauman, 2010).

Offender change research involves the evaluation of dynamic risk factors - often referred to as "criminogenic needs." These needs are factors related to criminal behaviour that are 
susceptible to change (Andrews \& Bonta, 2010). Increases or decreases in dynamic risk are associated with corresponding changes in reoffending behaviour (Andrews \& Bonta, 2010). For example, an offender who had previously disassociated with his antisocial peers is at a greater risk to reoffend if he starts socializing with these individuals again. Dynamic risk factors, therefore, serve as targets for intervention and risk reduction strategies.

Dynamic risk can be stable or acute. Stable risk factors are more durable, with change typically occurring over months or years (e.g., impulse control, attitudes toward authority; Bonta, 1996; Hanson \& Harris, 2000). Acute risk factors, on the other hand, are those that change rapidly, usually over hours or days (e.g., anger/hostility, negative mood; Bonta, 1996; Hanson \& Harris, 2000). Dynamic risk factors have been linked to a multitude of negative institutional and community outcomes. For instance, dynamic risk factors predict violence and serious misconducts — such as escape, smuggling, and inappropriate sexual behaviour - among incarcerated and hospitalized offenders (Hogan \& Olver, 2016; Van Voorhis et al., 2010; Wilson, Desmarais, Nicholls, Hart, \& Brink, 2013; Yang, Wong, \& Coid, 2010). Dynamic risk factors also predict rearrest, reimprisonment, and/or hospital readmission for IPV, violent, nonviolent, and sex offences among community-supervised offenders (Brem, Florimbio, Elmquist, Shorey, \& Stuart, 2018; Brown, et al., 2009; Feder \& Dugan, 2002; Greiner, Law, \& Brown, 2015; Hanby, 2013; Hilton \& Harris, 2005; McCoy \& Miller, 2013; Olver, Wong, Nicholaichuk, \& Gordon, 2007; Penney, Marshall, \& Simpson, 2016; Van Voorhis et al., 2010). The relationship between dynamic risk and negative institutional/community outcomes is true for men and women (Brown et al., 2009; Greiner et al., 2015; Van Voorhis et al., 2010; Wilson et al., 2013), as well as for offenders of different ethnicities (Hanby, 2013; Hogan \& Olver, 2016; Olver et al., 2007; Penny et al., 2016). 
It is clear that both static and dynamic risk factors are useful for predicting the likelihood of recidivism (e.g., Brown et al., 2009; Hanson et al., 2007; Helmus et al., 2012; Lewis et al., 2012; McCoy \& Miller, 2013; Philipse et al., 2006; Van Voorhis et al., 2010), however a further distinction between these variables can be made to demonstrate their utility in risk assessment. This distinction is between risk status and risk state (Douglas \& Skeem, 2005; Skeem \& Mulvey, 2002). Risk status is a baseline measure of risk determined by static factors, typically accompanied by a statistical estimate of recidivism risk (i.e., measured by actuarial risk scales comprised of static items; Douglas \& Skeem, 2005; Skeem \& Mulvey, 2002). It is relatively enduring due to the emphasis on static risk factors; therefore, is of little use when monitoring or treating offenders. A "high-risk" status, for example, indicates that intervention should be highintensity, but it does not identify targets for intervention. Given the dynamic nature of many important risk factors (e.g., antisocial cognitions, antisocial peers, substance abuse; Andrews \&

Bonta, 2010), effective intervention and case management require a more dynamic assessment of risk (Douglas \& Skeem, 2005). This type of assessment is known as risk state, a variable level of risk based largely on dynamic factors (Douglas \& Skeem, 2005; Skeem \& Mulvey, 2002).

\section{Incorporating Protective Factors}

It is well established that many negative factors contribute to an offender's increased risk to reoffend. However, in addition to risk factors, offenders may also experience a host of positive variables that mitigate their reoffending behaviour. These are referred to as protective factors. They represent internal strengths (e.g., prosocial identity) and external assets (e.g., social support) that shield the offender from the effects dynamic risk (Rutter, 1985; Ullrich \& Coid, 2011). The more protective factors one possesses, the more he or she will be shielded from risk (Lodewijks, de Ruiter, \& Doreleijers, 2010). It is important to note that protective factors are not 
simply the absence of risk, but can be viewed as the opposite of risk factors (e.g., having prosocial peers is protective, whereas having antisocial peers is a risk; Hawkins, Catalano, \& Miller, 1992; Webster, Martin, Brink, Nicholls, \& Middleton, 2004) or as separate entities existing without corresponding risk factors (e.g., optimism and hope; Woldgabreal, Day, \& Ward, 2016).

While there is a dearth of research on protective factors that are not inverted risk factors (Polaschek, 2016), both conceptualizations have been supported in the literature. Divergent validity analyses between risk and protective factor measures have yielded significant inverse correlations between the two constructs (e.g., see Abbiati, Azzola, Palix, Gasser, \& Moulin, 2017; Averill, 2016; Hanby, 2013; Persson, Belfrage, Fredriksson, \& Kristiansson, 2017; Smeth, 2013; Tamatea \&Wilson, 2009; Yesberg \& Polaschek, 2015). This supports the notion that protective factors are the opposite of risk factors. Woldgabreal and colleagues' (2016) work showed that protective factors that are not inverted risk factors predicted recidivism and technical supervision violations. Specifically, offenders with greater levels of psychological flexibility, general self-efficacy, optimism, and hope were less likely to recidivate or violate their supervision conditions (Woldgabreal et al., 2016). Evidence therefore suggests that protective factors exist both on a continuum of risk and as separate entities without corresponding risk factors.

Much of the research on protective factors and their effects on criminal behaviour has been conducted with juvenile offenders. This is likely due to the notion that the early identification of problem behaviors may prevent criminal or violent behavior later in life (Ullrich \& Coid, 2011). Further, protective effects are thought to be greater for adolescents than adults because they are still in the process of development (Ullrich \& Coid, 2011). Several studies 
found that protective factors reduced the risk of verbal, physical, and object-directed aggression, street drug use, and general, violent, and nonviolent recidivism among adolescents (Lodewijks et al., 2010; Richard, 2013; Sher, Warner, McLean, Rowe, \& Gralton, 2017; Viljoen et al., 2012). Further, protective factors present during youth have significantly predicted desistance from general offending in adulthood (Cox, Kochol, \& Hellund, 2018).

Many of the protective factor measures for adults that exist today were developed for specific offender subtypes (e.g., violent or sexual offenders) and have not typically been applied to representative samples of offenders. The remainder of the current section provides an overview of this research. Ulrich and Coid (2011) investigated the role of 15 protective factors in crime desistance among 800 adult male sexual and violent offenders supervised in the U.K. for an average of five years. Of these 15 hypothesized protective factors, social support, emotional support, spare time spent with prosocial family and friends, involvement in religious activities, and closeness with others significantly reduced the likelihood of violent reconviction. Spare time spent with prosocial family and friends also independently predicted violent recidivism after controlling for static risk level. Ulrich and Coid (2011) further argued that a focus on strengths in risk management and intervention programs may serve to enhance therapeutic alliance, promote recovery, motivate offenders, and reduce negative cognitions.

The Structured Assessment of Protective Factors for violence risk (SAPROF; de Vogel, de Ruiter, Bouman, \& de Vries Robbé, 2009, 2012) is a 17-item SPJ instrument designed to complement the Historical Clinical Risk Management-20 Version $2^{1}$ (HCR-20 ${ }^{\mathrm{V} 2}$; Webster, Douglas, Eaves, \& Hart, 1997) as a measure of protective factors for violence risk in adults. Its utility has been demonstrated in various jurisdictions and in both psychiatric and forensic

\footnotetext{
${ }^{1}$ Note that although the SAPROF was developed to complement the HCR-20 ${ }^{\mathrm{V} 2}$, it can also be used in conjunction with the original HCR-20 and the updated HCR-20 ${ }^{\mathrm{V} 3}$ (Douglas, Hart, Webster, \& Belfrage, 2013).
} 
samples. The SAPROF significantly predicted short- (one year) and long-term (11 years) violence cessation among 108 discharged male and female forensic psychiatric patients in the Netherlands (de Vries Robbé, de Vogel, Douglas, \& Nijman, 2015). A recent study by Haines and colleagues (2018) investigated the predictive validity of the SAPROF in 151 psychiatric inpatients in the U.K. The SAPROF Internal (comprising intelligence, secure attachment in childhood, empathy, coping, and self-control items) and Motivation subscales (comprising work, leisure activities, financial management, motivation for treatment, attitudes towards authority, life goals, and medication items) were significantly associated with reduced violence at the sixmonth follow-up (Haines et al., 2018). In a study on 193 male and female forensic psychiatric inpatients in Stockholm, the SAPROF significantly predicted desistance from actual and threatened violence over a one-year follow-up period (Persson et al., 2017). Further, predictive accuracy was greater for the subtraction of the SAPROF score from the HCR-20 ${ }^{\mathrm{V} 3}$ (i.e., risk) score than either SAPROF or HCR-20 ${ }^{\mathrm{V} 3}$ scores alone, suggesting that risk assessments should consider both risk and protective factors (Persson et al., 2017).

In addition to forensic psychiatric samples, the SAPROF has been validated in purely forensic samples as well. For example, the predictive validity of the SAPROF was examined among 277 sex offenders against children followed for an average of 5.7 years in Austria (Turner et al., 2016). Turner and colleagues (2016) found the SAPROF to significantly predict abstention from general recidivism, however, it did not significantly predict abstention from violent or sexual recidivism. The SAPROF demonstrated further predictive utility in forensic settings in a study on 450 male sexual offenders followed for an average of 5.8 years in Austria (Yoon et al., 2018). The SAPROF Total score, as well as self-control, work, financial management, and life goals items significantly predicted desistance from general, nonsexual violent, and violent 
(including sexual) recidivism, and the secure attachment item significantly predicted desistance from sexual recidivism.

Other researchers have assessed the predictive validity of both the SAPROF and the Short-Term Assessment of Risk and Treatability (START; Webster, Martin, Brink, Nicholls, \& Desmarais, 2009; Webster et al., 2004), a 20-item SPJ instrument with Strength and Vulnerability domains. The START was designed to aid in the prediction of violence, suicide, self-harm, self-neglect, unauthorized absence, substance abuse, and victimization risk in adults with mental, personality, and substance related disorders. In a study of 100 male and female psychiatric inpatients in Dublin followed for six months, the SAPROF significantly predicted reduced violence and self-harm (Abidin et al., 2013). The START Strength domain significantly predicted reduced violence as well, but it failed to predict reduced self-harm (Abidin et al., 2013). In a more recent study on 152 hospitalized male sexual offenders in the U.S., START Strength scores significantly predicted desistance from any, verbal, and physical aggression toward others over a 90-day period (Cartwright, Desmarais, Hazel, Griffith, \& Azizian, 2018). Strength scores also significantly predicted cessation from any and verbal aggression over a 180day period (Cartwright et al., 2018).

Research by Lowder and colleagues provides additional evidence for the predictive utility of the START Strength domain in forensic samples. In a study on 550 mentally-disordered male and female offenders in jail-diversion programs in the U.S., START Strength scores were significantly inversely related with number of arrests and number of jail days over a one-year period (Lowder, Desmarais, Rade, Coffey, \& Van Dorn, 2017). These results were replicated in a sample of 95 male and female jail-diversion offenders in the U.S. for 3-, 6-, 9-, 12-, and 18month follow-up periods (Lowder, Desmarais, Rade, Johnson, \& Van Dorn, 2017). 
The instruments for assessing protective factors in adults discussed thus far were developed for specific offender subtypes or outcomes. However, one scale that was developed for use with representative samples of offenders to predict general offending is the Inventory of Offender Risk, Needs, and Strengths (IORNS; Miller, 2006a). The IORNS is a 130-item selfreport scale that assesses static and dynamic risk, as well as protective factors in adults. The initial evaluation of the IRONS included 162 male general offenders residing in a half-way house in the U.S. (Miller, 2006b). This study showed that offenders who violated half-way house rules multiple times during the 15-month follow-up period had significantly fewer self-reported protective factors than those who did not have multiple rule violations (Miller, 2006b). A later IORNS validation study on 110 adult male sexual offenders in the U.S. revealed that selfreported protective factors significantly predicted abstention from general, sexual, and violent recidivism over six years (Miller, 2015). Moreover, self-reported protective factors predicted abstention from sexual reoffending during the six-year follow-up period while controlling for overall static and dynamic risk (Miller, 2015).

Protective factors have been shown to predict general, sexual, violent (both including and excluding sexual violence), and nonviolent recidivism in adults, as well as threatened violence. They have also demonstrated utility in predicting general, violent (including verbal, physical, and object-directed), and nonviolent recidivism, as well as street drug use among adolescents. The significant inverse relationship between protective factors and adverse outcomes has held true in several jurisdictions and with variable follow-up times for both young and adult offenders. Taken together, these findings illustrate the important role protective factors play in crime desistance. Given the potential that protective factors have shown in the management and prevention of criminal behaviour, steps have been taken to develop a more balanced and well- 
rounded approach to risk assessment that considers offenders' strengths as well as their weaknesses (de Ruiter \& Nicholls, 2011). This includes incorporating protective factors into risk assessment instruments (Serin, 2007, 2015, 2017).

The inclusion of protective factors (e.g., quality marriage, stable employment, prosocial relationships; Andrews, 1980; Benda, 2005; Laub, Nagin, \& Sampson, 1998; Maume, Ousey, \& Beaver, 2005; Uggen, 1999, 2000) in risk assessment allows decision makers to move from the identification of risk to the reduction of risk by promoting desistance factors (Serin, Lowenkamp, \& Lloyd, in press). The process of desistance is distinct from the initiation and persistence of crime (Serin \& Lloyd, 2009; Stouthamer-Loeber, Wei, Loeber, \& Masten, 2004). Namely, offenders must enhance their skills and strengths in positive ways to help them move away from crime. The desistance aspect of offender supervision, therefore, emphasizes working with offenders to build skills and utilize their strengths. Moreover, it facilitates positive interactions with offenders to promote prosocial changes, rather than limiting supervision strategies to the prevention of criminal behaviour (Serin, 2016).

\section{Scale Validation}

The phenomena examined in psychological research are often unobservable. These phenomena are referred to as latent constructs, and they can be measured using scales comprised of different variables that reflect the target construct(s). Measuring the degree of correspondence between what an instrument is designed to measure and what it actually measures - also known as construct validity - is integral to psychological research; a study's conclusions have questionable meaning if the measures used are not valid (Flake, Pek, \& Hehman, 2017). In correctional research for instance, we cannot accurately predict future criminal behaviour or use risk ratings for case planning if risk tools are not valid. Flake and colleagues (2017) provide an 
overview of the scale validation process (i.e., the process by which scales are examined to determine if they accurately reflect the target construct), summarized below.

Construct validation involves three phases: substantive, structural, and external. The substantive component is similar to, and was previous called, content validity (Loevinger, 1957). It is concerned with the theoretical underpinnings of a scale and uses previous literature to support construct operationalization, scope, and item selection. Beyond literature reviews, methods such as expert reviews and focus groups can be used to select items and examine content relevance and representativeness. For example, scale developers can invite experts in the field (e.g., authors of relevant research) to judge how well the items reflect the target construct and whether important variables have been omitted (Gehlbach \& Brinkworth, 2011). Experts' opinions can be evaluated individually or through focus groups. For the latter, the content of the scale is valid if there is consensus among group members that the items accurately reflect the target construct (e.g., Salerno, Marshall, \& Picken, 2012).

The structural phase of construct validation involves an examination of the scale's psychometric properties using quantitative analyses. This includes measures such as internal consistency (e.g., Cronbach's alpha and item-total correlations), test-retest reliability, inter-rater reliability, and factor analysis. Internal consistency estimates measure the extent to which items represent the same construct. Scales that have high internal consistency are said to be reliable measures of the target construct. Other reliability measures, such as test-retest and inter-rater reliability determine the consistency of ratings from one time to another (test-retest) and across evaluators for the same individual (inter-rater). Factor analysis is used to understand a scale's underlying structure by examining how items are related (Tabachnick \& Fidell, 2013). 
Finally, evidence for how the scale's constructs relate to other constructs or predict certain outcomes (i.e., criterion-referenced validity; Helmus \& Babchishin, 2017) is examined in the external phase of construct validation. Methods used at this level include convergent (congruent) and divergent (discriminant) validity analyses, as well as tests of criterion-referenced validity, including predictive, concurrent, and retrospective validity (American Psychological Association [APA], 2018). Convergent validity is the extent to which the scale under question is strongly related to theoretically similar measures (APA, 2018). In contrast, divergent validity is the degree to which the scale diverges from theoretically dissimilar measures (APA, 2018). Convergent and divergent validity can be examined using correlational analyses, with convergent validity evidenced by strong positive correlations and divergent validity evidenced by low or strong negative correlations.

As mentioned above, there are three types of criterion-referenced validity: predictive, concurrent, and retrospective. Predictive validity measures the association between scale scores and a future event (e.g., whether a measure of criminal behaviour accurately predicts future offending; APA, 2018). Concurrent validity measures the association between scale scores and a related measure obtained at approximately the same time (e.g., whether a measure of criminal behaviour can accurately predict current criminal activity; APA, 2018). Retrospective validity measures the association between a scale designed to capture a certain behaviour and past occurrences of this behaviour (e.g., whether a measure of criminal behaviour can accurately predict previous offences; APA, 2018). Note that retrospective validity is distinct from retrospective predictive validity. In the context of risk assessment, retrospective predictive validity would involve obtaining a sample of offenders, retrospectively rating them on some 
instrument, and using these scores to predict future criminal behaviour. Retrospective validity, in contrast, would involve predicting criminal history based on current scale scores.

Risk assessment instruments are criterion-referenced scales, as opposed to normreferenced scales, which aim to measure a particular construct (e.g., intelligence, personality, ability; Helmus \& Babchishin, 2017). This means they are intended to predict a specific outcome, and traditional elements of scale validation are not applicable (Helmus \& Babchishin, 2017). In their review on evaluating the predictive accuracy of risk assessment tools, Helmus and Babchishin (2017) argue that the predictive accuracy and efficient administration of risk tools are maximized by measuring distinct constructs using as few items as possible, which works to reduce internal consistency. Having high internal consistency may, therefore, impinge on the ability of a risk assessment scale to accurately predict recidivism (Helmus \& Babchishin, 2017). Moreover, having a small number of items prevents researchers from conducting reliable factors analyses, calling into question examinations of risk scales' factor structure (Helmus \& Babchishin, 2017). In their review, Helmus and Babchishin (2017) contend that evidence of predictive validity alone often legitimizes the use of risk assessment scales.

Given the criticisms regarding the application of classic scale validation techniques (e.g., internal consistency, factor structure) to risk assessment instruments, it is important to acknowledge that not all tools used for risk prediction were designed specifically for this purpose. Some correctional tools were designed for case management purposes to inform decisions that are intended to maximize optimal outcomes for offenders. These tools are used, first and foremost, to aid case planning and risk management. They are designed to assist supervision officers in the identification of factors related to increased risk for offenders on their caseloads, and to create individualized plans of care to address these factors (i.e., the case 
planning element). This ultimately serves to reduce offenders' likelihood of reoffending (i.e., the risk management element). Case management tools measure constructs related to risk that are targeted in case planning (e.g., criminal orientation, impulsivity, destabilizers, prosocial identity). Thus, having items that hang together, representing target constructs, has utility in practice. In other words, internal consistency and factor structure are relevant to the construct validation of correctional instruments that were not designed solely to assess risk (e.g., structured case management assessments).

\section{DRAOR Development and Implementation}

The Dynamic Risk Assessment for Offender Re-Entry (DRAOR) is a 19-item structured case management scale designed to assist community parole officers (POs) in the assessment of offender risk throughout supervision (DRAOR; see Appendix A; Serin, 2007, 2015, 2017 2,3 ). Specifically, the DRAOR allows community POs to assess their client at every supervision contact, helping them create individualized case plans and risk management strategies based on real-time changes in offender risk.

Recall that static scales measure risk status (one's level of risk in relation to others at a single point in time), while dynamic scales assess risk state (case-specific changes in risk). As the DRAOR — a dynamic scale — focuses on risk state, it aids POs in the assessment of individual variations in risk. That is, it serves as a tool for differentiating offenders within risk groups. High-risk offenders present with different sets of dynamic risk and protective factors than lowrisk offenders, for example. The DRAOR distinguishes between these offenders, determining who is at higher risk to reoffend and when.

\footnotetext{
${ }^{2}$ Supervision officers completed DRAOR assessments for the current sample between March, 2011 and July, 2011 using the 2007 version of the scoring manual.

${ }^{3}$ An early version of the user-manual was developed by Serin, Mailloux, and Wilson (2012).
} 
The DRAOR evaluates psychosocial and contextual variables across Stable, Acute, and Protective domains, incorporating both the nature of association between items and recidivism (i.e., risk factors versus protective factors) and the degree of stability among risk factors (i.e., stable versus acute risk factors). As previously discussed, stable risk factors are susceptible to change — which typically occurs over months or years — and are associated with recidivism risk. The six Stable items reflect attitude (2), trait (2), and behaviour patterns (2). Attitude items include attitudes towards authority (defiant attitudes toward those in authority) and sense of entitlement (self-centeredness). Trait items include impulse control (acting without forethought) and problem solving (poor ability to find prosocial solutions to problems). Behaviour items include peer associations (spending time with antisocial others) and attachments with others (social disconnection or problematic interpersonal attachments).

Acute risk factors are also changeable factors associated with recidivism risk, but change occurs more rapidly than with stable risk factors, usually taking place over hours or days. The seven Acute items include situation (4), mood (2), and behaviour (1) factors. Situation items include opportunity/access to victims (opportunity to offend), employment (unemployed), interpersonal relationships (problems in close interpersonal relationships), and living situation (lack of accommodations). Mood items include anger/hostility (irritability or rage/behaving antagonistically toward others) and negative mood (depressed or anxious mood). The behaviour item captures substance abuse (use of illegal drugs and substances banned by supervision order).

Protective factors are internal and external variables that may reduce the risk of reoffending. The DRAOR Protective domain comprises strengths that exist without corresponding risk factors (as opposed to strengths that are inverted risk factors), with the six items reflecting prosocial perceptions (3) and prosocial connectedness (3). Prosocial perceptions 
items include responsive to advice (open to guidance from prosocial others), prosocial identity (views self as oriented toward non-criminal goals), and costs/benefits (views prosocial options more favorably than criminal actions). Social connectedness items include high expectations (high sense of hope in ability to make prosocial changes), social support (availability of prosocial others), and social control (internal investment in prosocial goals approved by others; see Appendix B for a more detailed description of items).

DRAOR items are scored on a 3-point scale (0-2). For Stable and Acute risk items, a score of 0 indicates the factor is currently absent for the offender, a score of 1 is assigned if the factor poses a slight risk, or if it is unclear due to mixed evidence, and a score of 2 indicates the factor is a definite problem. For Protective items, a rating of 0 suggests the factor is not currently protective for the offender, a rating of 1 indicates the factor is slightly protective, or it is unclear due to mixed evidence, and a rating of 2 suggests the factor is a definite asset. DRAOR Total scores are calculated by summing the Stable and Acute domains, and subtracting the Protective domain. Total scores range from -12 to 26 , with lower scores reflecting lower risk and a greater number of protective factors and higher scores reflecting higher risk and fewer protective factors. Scale items are also examined to provide a summary of potential targets for intervention.

Given their relationship to recidivism, increases or decreases in DRAOR item scores should translate into changes in case planning by officers. Recently, Serin and Chadwick (2017) developed DRAOR cut-off scores to provide officers with guidelines for adjusting supervision intensity based on the scale's dynamic risk and desistance correlates. Cut-off scores were determined through a visual inspection of the data. Offenders can be categorized into one of four risk levels based on these scores (Low, Moderate, Moderate/High, High; see Appendix A), 
which will allow evaluators to utilize DRAOR Total scores to modify supervision level that is based on static risk assessments done at the start of supervision.

Serin and Chadiwick's (2017) proposed guidelines suggest that offenders' level of supervision should decrease by one level when DRAOR Total scores are Low, increase by one level when DRAOR Total scores are Moderate/High or High, and remain the same when DRAOR Total scores are Moderate. For example, if the recommended level of supervision based on the Iowa Risk Assessment (IRA) is High Normal (see the Measures section for a description of the IRA and its supervision levels), but the DRAOR Total score is Low, the offender's supervision level should decrease from High Normal to Low Normal. Conversely, if the recommended level of supervision based on the IRA is High Normal, but the DRAOR Total score is Moderate/High or High, the offender's supervision level should increase from High Normal to Intensive. If the DRAOR Total score is Moderate, the supervision level should remain the same.

The DRAOR has been administered to over 6000 male and female communitysupervised offenders in New Zealand and Iowa. It has been utilized to predict recidivism among high-risk offenders (Yesberg \& Polaschek, 2015), sex offenders (Averill, 2016; Smeth, 2013), and samples representing diverse offender-types, including violent, nonviolent, and sex offenders (Chadwick, 2014; Hanby, 2013; Serin et al., 2016; Tamatea \& Wilson, 2009; Yesberg, Scanlan, Hanby, Serin, \& Polaschek, 2015). Validation results from these studies are summarized below.

Content validity. The DRAOR was developed based on theory, informed by the correctional, desistance, re-entry, and rehabilitation literatures (see the Measures section for a review of the instrument's development). Items in the Stable domain address criminal orientation and impulsivity concerns, items in the Acute domain address destabilizers and lifestyle stressors, 
and items in the Protective domain address social support and prosocial identity changes.

Content relevance was determined through a review of the aforementioned literatures, with item selection based primarily on the following works: Andrews and Bonta (2010), Hanson and Harris (2000), Hanson et al. (2007), Maruna (2001), and Sampson and Laub (2005).

Factor structure. As previously mentioned, the DRAOR has three theoretically meaningful domains: Stable, Acute, and Protective. The factor structure of the DRAOR has been examined in an exploratory fashion in three studies to date. In a large study containing over 97,000 DRAOR assessments from a representative sample of all male and female parolees in New Zealand $(N=3,498)$, an Exploratory Factor Analysis (EFA) suggested that three factors be retained (Hanby, 2013). These factors were Mostly Stable, Mostly Acute, and Protective, comprising 17 of the 19 original DRAOR items (interpersonal relationships and employment were dropped). The total variance explained by this model was $38.2 \%$. Using a representative sample of all male and female probationers and parolees in the $5^{\text {th }}$ District of Iowa $(N=391)$, Chadwick (2014) found support for a two-factor structure of the DRAOR through an EFA. This included a Risk and Protective domain containing all 19 original DRAOR items. Lastly, a Principal Components Analysis (PCA) using a sample of 299 high-risk male offenders on parole in New Zealand (Yesberg \& Polaschek, 2015) yielded four domains: Stable, Internal Acute, External Acute, and Protective. This model retained all 19 original DRAOR items and explained $51.3 \%$ of the variance.

Several researchers have carried out Confirmatory Factor Analyses (CFAs) to examine whether the original factor structure of the DRAOR, as well as factor structures obtained through EFA or PCA adequately fit their data. As the models obtained through EFA and PCA varied across studies, the following discussion concerns the original three-factor structure of the 
DRAOR only. Hanby (2013) examined the adequacy of model fit using three criteria: (1) Goodness of Fit Index (GFI) greater than .90, (2) Comparative Fit Index (CFI) greater than .90; and (3) Root Mean Square Error of Approximation (RMSEA) less than .06. The original threefactor structure of the DRAOR was appropriate for use with her representative sample of New Zealand parolees $(\mathrm{GFI}=.98$ for middle and last DRAOR assessments; $\mathrm{CFI}=.93$ and .94 for middle and last assessments, respectively; RMSEA $=.05$ for middle and last assessments). A CFA of the original three-factor structure of the DRAOR on a sample of high-risk offenders, however, revealed a poor fitting model $(\mathrm{CFI}=.84$; RMSEA $=.08$; Yesberg \& Polaschek, 2015).

Reliability. Tamatea and Wilson (2009) were the first to examine the psychometrics of the DRAOR. Their study included a representative sample of 59 male and female offenders in New Zealand followed for one year while on probation. These researchers found that the DRAOR subscale scores were normally distributed and, as expected, offenders' scores demonstrated change throughout supervision, reflecting changes in dynamic risk.

In Hanby’s (2013) study on 3,498 general New Zealand parolees, internal consistency estimates_as measured by Cronbach's alpha—were acceptable ${ }^{4}$ for the Stable $(\alpha=.81)$ and Protective subscales $(\alpha=.84)$, but below the acceptable level for the Acute subscale $(\alpha=.62)$. Item-total correlations were acceptable $\mathrm{e}^{5}$ for the Stable $(r=.47$ to .63$)$ and Protective subscales $(r$ $=.52$ to .57 ), and generally acceptable for the Acute subscale, with only the interpersonal relationships and employment item-total correlations below the acceptable level $(r=.20$ to .46).

Chadwick (2014) reported similar results in his validation study using a representative sample of 391 probationers and parolees in Iowa. Specifically, Cronbach's alphas were acceptable for the Stable $(\alpha=.81)$ and Protective subscales $(\alpha=.86)$, but below the acceptable

${ }^{4}$ Cronbach's alphas of .70 (Reynolds \& Livingstone, 2012) or .80 (Clark \& Watson, 1995) and above indicate acceptable internal consistency.

${ }^{5}$ Item-total correlations of .30 and above indicate acceptable internal consistency (Field, 2009). 
level for the Acute subscale $(\alpha=.62)$. Item-total correlations from Chadwick's study were acceptable for the Stable ( $r=.43$ to .68$)$ and Protective subscales ( $r=.57$ to .68 ), and generally acceptable for the Acute subscale ( $r=.28$ to .42 ), with only the substance abuse item-total correlation below the acceptable level. These three validation studies provide initial moderate support for the reliability of the DRAOR.

Convergent and divergent validity. Tamatea and Wilson (2009) examined the convergent and divergent validity between the Risk of re-Conviction X Risk of re-Imprisonment (RoC*RoI), a validated static risk assessment model used in New Zealand (Bakker, O’Malley, \& Riley, 1998), and the DRAOR subscales using a sample of 59 probationers. The RoC*RoI was positively, but not significantly, correlated with the Stable $(r=.22)$ and Acute $(r=.03)$ subscales and significantly negatively correlated with the Protective subscale $\left(r=-.33\right.$, a moderate effect $\left.{ }^{6}\right)$. In a comprehensive study of 3,498 offenders, Hanby (2013) also examined the convergent and divergent validity between the RoC*RoI and the DRAOR - both at initial and last assessments prior to recidivism or the study end date. The RoC*RoI demonstrated small to moderate significant correlations in the expected directions with the DRAOR Total and the Stable, Acute, and Protective subscales at initial $(r=.32, .31, .24,-.25$, respectively) and last assessments $(r=$ $.37, .35, .30,-.31$, respectively). In a recent study examining the validity of the DRAOR among 851 male sex offenders on parole in New Zealand, Averill (2016) reported small to moderate significant associations in the expected directions between the RoC*RoI and the DRAOR Total $(r=.38)$ and the Stable $(r=.37)$, Acute $(r=.40)$, and Protective domains $(r=-.25)$.

The DRAOR has demonstrated convergent and divergent validity with other static risk tools, such as the Static-99R (an actuarial risk instrument used to measure sexual recidivism risk; Phenix, Fernandez, Harris, Helmus, Hanson, \& Thornton, 2016). Using a sample of 193 male sex 
offenders under community supervision in Iowa, Smeth (2013) reported small significant positive correlations between the Static-99R and the DRAOR Total scale $(r=.26)$, and Stable $(r$ $=.23)$ and Acute subscales $(r=.17)$, as well as a small significant negative correlation between the Static-99R and the Protective subscale $(r=-.24)$. The DRAOR also demonstrated significant associations with is the Automated Sexual Recidivism Scale (ASRS; Skelton, Riley, Wales, \& Vess, 2006), a static risk tool used in New Zealand for measuring sexual recidivism risk. The ASRS was moderately positively correlated with the DRAOR Total scale $(r=.47)$, and Stable $(r$ $=.45)$ and Acute subscales $(r=.45)$, and moderately negatively correlated with the Protective subscale $(r=-.37)$ in a study of 851 sex offenders (Averill, 2016).

Yesberg and Polaschek (2015) examined the convergent and divergent validity between the DRAOR and the Violence Risk Scale (VRS; a static and dynamic measure of violence and general risk; Wong \& Gordon, 1999-2003) using a sample of 299 high-risk New Zealand parolees. The VRS Static domain significantly positively correlated with DRAOR Total $(r=.16$, a small effect), but not the Stable $(r=.08)$ or Acute subscales $(r=.12)$, and significantly negatively correlated with the Protective subscale ( $r=-.19$, a small effect). The Dynamic VRS domain demonstrated small significant positive associations with the DRAOR Total scale $(r=$ $.25)$, and Stable $(r=.18)$ and Acute subscales $(r=.22)$, and small significant negative associations with the Protective subscale $(r=-.20)$. Yesberg and Polaschek (2015) also found small to moderate significant correlations in the expected directions between the Release Proposal Feasibility Assessment-Revised (RPFA-R; a dynamic measure of preparedness for release; Wilson, 2011) and the DRAOR Total $(r=.30)$ and the Stable $(r=.20)$, Acute $(r=.34)$ and Protective domains $(r=-.17)$. 
Several authors have also examined associations between the DRAOR Total and subscale scores. This includes the authors mentioned above, as well as Ferguson $(2015)^{7}$ and Muirhead $(2016),{ }^{8}$ who assessed inter-correlations between DRAOR scale and subscale scores in overlapping samples of adolescent and adult male and female offenders under community supervision in New Zealand ( $N=244$ and 369, respectively). Correlations were significant and in the expected directions. The DRAOR Total was strongly correlated with the Stable $(r=.87$ to .92), Acute ( $r=.81$ to .85 ), and Protective subscales ( $r=-.82$ to -.89; Averill, 2016; Ferguson, 2015; Muirhead, 2016; Smeth, 2013). Additionally, the DRAOR Stable and Acute scores were strongly positively related $(r=.52$ to .72$)$, indicating these subscales both measure dynamic risk (Averill, 2016; Ferguson, 2015; Muirhead, 2016; Smeth, 2013; Tamatea \& Wilson, 2009). The Protective subscale showed moderate to strong negative associations with the Stable ( $r=-.46$ to .73 ) and Acute subscales ( $r=-.41$ to -.63$)$, suggesting this subscale measures factors that operate differently from dynamic risk factors (Averill, 2016; Ferguson, 2015; Muirhead, 2016; Smeth, 2013; Tamatea \& Wilson, 2009).

Overall, the DRAOR Total, Stable, and Acute scales demonstrated small to moderate significant correlations with several validated static and dynamic risk assessment instruments in the positive direction, including the RoC*RoI, Static-99R, ASRS, VRS (note that Stable and Acute subscales did not significantly correlate with the VRS Static domain), and the RPFA-R. The Stable subscale functions similarly to a risk-need scale, identifying traditional criminogenic needs for intervention. The Acute subscale identifies risk factors for failure and can be used to refine case planning and risk management approaches. The pattern of small to moderate

\footnotetext{
${ }^{7}$ Correlations are based on proximal DRAOR assessments for general recidivism among both youth and adults (see Table 8 in Ferguson, 2015).

${ }^{8}$ Correlations are based on proximal DRAOR assessments for general among adolescents (see Table 8 in Muirhead, 2016).
} 
significant negative correlations between the Protective subscale and the static and dynamic measures of risk suggests that protective factors and risk factors operate differently.

Predictive validity. In the original pilot study assessing 59 general offenders, Tamatea and Wilson (2009) examined predictive validity by comparing recidivists with non-recidivists using a series of one-way independent $t$-tests. The Stable and Acute subscales did not distinguish between recidivists and non-recidivists. The Protective subscale, on the other hand, significantly predicted (1) reoffence and/or breaches of parole conditions, (2) convictions for any offence, and (3) recalls to prison (excluding breaches of parole conditions), ${ }^{9}$ but not breaches of parole conditions alone. This suggests the Protective subscale is a significant predictor of parole success, but not supervision compliance.

In her comprehensive study containing over 97,000 DRAOR assessments $(N=3,498)$, Hanby (2013) used the assessment most proximal to the recidivism event to examine the predictive validity of DRAOR Total and subscale scores. Her analyses were conducted for the overall sample, as well as separately for Māori offenders. As effect sizes were significant and comparable for both groups, however, the following discussion pertains to the overall sample. Hanby reported moderate to large ${ }^{10}$ AUCs for any reconviction (including criminal and administrative offences), ranging from .66 to .72 and moderate AUCs for criminal reconvictions, ranging from .62 to .67. Chadwick (2014) did not find the DRAOR Total or subscales to be significant predictors of general or violent recidivism among probationers and parolees in Iowa $(N=391)$; however, they did significantly predict technical violations (AUCs were moderate,

\footnotetext{
${ }^{9}$ In New Zealand, offenders may be recalled to prison if, for example, the Parole Board is concerned about undue risk without a breach conviction.

${ }^{10} \mathrm{As}$ a rough heuristic, an AUC of .56 corresponds to a small effect size, .64 reflects a moderate effect, and .71 reflects a large effect size, as these values roughly correspond to Cohen's $d$ s of $.20, .50$, and .80 (see Rice \& Harris 2005).
} 
ranging from .57 to .61). The null findings for general and violent recidivism could be due to the small number of recidivists $\left(n_{\text {general }}=32, n_{\text {violent }}=11\right)$. While AUCs are robust to attenuations in magnitude due to high or low base rates (Babchishin \& Helmus, 2016), 100 events and 100 nonevents are recommended for adequate statistical power when predicting dichotomous outcomes in logistic regression (Vergouwe, Steyerberg, Eijkemans, \& Habbema, 2005), and similar standards would likely apply to AUCs (L. M. Helmus, personal communication, January 25, 2018).

Yesberg and Polaschek (2015) investigated the utility of the DRAOR in predicting breaches of parole conditions, new convictions (i.e., excluding those due to breaches), new violent convictions, and reimprisonment (a breach or new offence that led to a new sentence of imprisonment) among 299 high-risk offenders. No significant effect sizes were found for new violent convictions and only the Protective subscale significantly predicted breaches of parole conditions $(\mathrm{AUC}=.58$, a small effect). The DRAOR Total scale, and Stable, Acute, and Protective subscales did, however, significantly predict new convictions (AUCs were moderate, ranging from .57 to .62), as well as reimprisonment (AUCs were moderate, ranging from .59 to $.62)$.

Additional work by Yesberg and colleagues (2015) evaluated whether the DRAOR predicted general recidivism for both male $(n=133)$ and female $(n=133)$ parolees in New Zealand, and whether there were differences in predictive accuracy between genders. This sample is a subset of the dataset used by Hanby (2013), representing all offender types. Using Cox regression survival analysis (which controls for different follow-up lengths among offenders), the researchers found that the DRAOR Total significantly predicted time to recidivism for both men and women. Analyses also revealed that, together, the Stable, Acute, and 
Protective subscales significantly predicted time to recidivism for women, but not for men. Further, only the Acute subscale made a statistically significant unique contribution to the prediction of time to recidivism for women, suggesting it is responsible for much of the DRAOR's predictive utility among this population. None of the subscales made a statistically significant unique contribution to the prediction of time to recidivism for men.

In a study on 193 sex offenders, neither the DRAOR Total, nor the subscales significantly predicted sexual recidivism (Smeth, 2013). Total and subscale scores were moderate significant predictors of parole violations, however $(\mathrm{AUCs}=.61$ to $.69 ;$ Smeth, 2013). Further research provided more promising use for the DRAOR among sex offenders. Specifically, using univariate Cox regression survival analyses, Averill (2016) reported significant independent associations between the DRAOR Total scale, and the Acute and Protective subscales with time to sexual recidivism $(N=851)$. When the Stable, Acute, and Protective subscales were examined together, they significantly predicted time to sexual recidivism, but no subscale made a unique contribution to this model. Results were better for violent and general recidivism, with the DRAOR Total scale, and the Stable, Acute and Protective subscales all independently predicting time to both recidivism outcomes. The three subscales significantly predicted time to both violent and general recidivism when entered in the model together; however, none of the subscales added unique predictive utility to time to violent recidivism, and only the Protective subscale uniquely predicted time to general recidivism.

Serin et al. (2016) recently investigated the utility of the DRAOR in predicting recidivism among a representative sample of all probationers and parolees in Iowa $(N=563)$. The Total and subscale scores were moderate to strong predictors of technical parole violations (AUCs $=.66$ to 
$.71)$, moderate to strong predictors of serious parole violations (AUCs $=.66$ to .72 ), and moderate predictors of new crimes (AUCs $=.67$ to .70$)$.

The predictive validity of the DRAOR has also been examined among adolescent offenders in two studies on New Zealand samples. Ferguson (2015) examined the predictive validity of initial and proximal DRAOR scores among adolescent (aged 17-19) and adult (aged 20-60) male offenders under community supervision. For adolescents, initial DRAOR Total scores significantly predicted criminal reconvictions $(N=122 ; \mathrm{AUC}=.64$, a moderate effect $)$, but none of the initial DRAOR subscale scores demonstrated significant predictive accuracy for this outcome. Neither initial DRAOR scale or subscale scores significantly predicted reconvictions due to breaches of conditions $(N=101)$. Proximal DRAOR Total and subscale scores significantly predicted both criminal $(N=122)$ and breach reconvictions $(N=101$; AUCs were moderate to large, ranging from .65 to .74 and .65 to .72 , respectively). For adults, initial DRAOR Total and Acute scores significantly predicted criminal reconvictions $(N=122$; AUCs $=$ .62 and .63 , respectively, small effects $)$ and breach reconvictions $(N=101$; AUCs $=.66$ and .71 , respectively, moderate to large effects), but the Stable and Protective domains did not. Proximal DRAOR Total, Stable, and Acute scores significantly predicted criminal reconvictions $(N=122$; AUCs were small to moderate, ranging from .63 to .65) and breach reconvictions $(N=101$; AUCs were moderate to large, ranging from .68 to .77), but the Protective domain did not. Muirhead (2016) investigated the predictive validity of the DRAOR among male and female adolescent offenders (aged 17 to 19) under community supervision. Note that this sample overlaps with Ferguson (2015) and includes female youth. Initial DRAOR Total and subscale scores significantly predicted any criminal $(N=369)$ and violent reconvictions $(N=397$; AUCs were moderate, ranging from .59 to .63 for both outcomes). Proximal DRAOR Total and 
subscale scores significantly predicted any criminal reconvictions $(N=369$; AUCs were moderate to large, ranging from .68 to .73) and violent reconvictions $(N=397$; AUCs were small to moderate, ranging from .61 to .68).

The DRAOR can be applied to samples of men and women, adults and adolescents, different ethnicities, and diverse offender types. A summary of the predictive validity results described in this section is presented in Table 1. Total scores have significantly predicted any recidivism (including technical violations; $100 \%$ of the effect sizes were significant), general recidivism (excluding technical violations; $89 \%$ of the effect sizes were significant), violent recidivism ( $67 \%$ of the effect sizes were significant), and supervision violations $(89 \%$ of the effect sizes were significant). Significant effects ranged from small to large for any recidivism, general recidivism, and supervision violations, and small to moderate for violent recidivism. The evidence for the DRAOR as an accurate predictor of sexual recidivism risk is mixed; AUCs and correlations were nonsignificant, but Averil (2016) reported promising results for the use of the DRAOR in this context using Cox regression survival analyses. Additionally, the null findings in Smeth's (2013) study may be due to the small number of sexual recidivists $(n=16)$ in her sample. Further investigation into the use of the DRAOR as a predictive tool for sexual recidivism is therefore warranted. 
Table 1

DRAOR Predictive Validity Results (Point-Biserial Correlations and AUCs) Reported in Previous Research

\begin{tabular}{|c|c|c|c|c|c|c|c|c|c|c|}
\hline \multirow[b]{2}{*}{ Source } & \multirow[b]{2}{*}{$N$} & \multirow[b]{2}{*}{ Follow-up ${ }^{\mathrm{a}}$} & \multicolumn{2}{|r|}{ Stable } & \multicolumn{2}{|r|}{ Acute } & \multicolumn{2}{|c|}{ Protective } & \multicolumn{2}{|r|}{ Total } \\
\hline & & & $r$ & AUC $[95 \% \mathrm{CI}]$ & $r$ & AUC $[95 \% \mathrm{CI}]$ & $r$ & $\mathrm{AUC}[95 \% \mathrm{CI}]$ & $r$ & AUC $[95 \% \mathrm{CI}]$ \\
\hline \multicolumn{11}{|c|}{ Any recidivism (including supervision violations) } \\
\hline Hanby $(2013)^{b}$ & 1775 & 2 years & - & $.65[.62, .67]$ & - & $.70[.68, .72]$ & - & $.65[.62, .67]$ & - & $.69[.67, .72]$ \\
\hline Hanby $(2013)^{c}$ & 3372 & 2 years & - & $.66[.65, .68]$ & - & $.72[.70, .74]$ & - & $.67[.65, .68]$ & - & $.71[.69, .73]$ \\
\hline Chadwick $(2014)^{\mathrm{d}}$ & 391 & $M=250$ days & - & $.62[.56, .67]$ & - & $.59[.54, .65]$ & - & $.58[.52, .66]$ & - & $.62[.56, .68]$ \\
\hline Yesberg \& Polaschek $(2015)^{\mathrm{e}}$ & 299 & 6 months & - & $.59 *[-,-]$ & - & $60^{*}[-,-]$ & - & $.62 * *[-,-]$ & - & $.62 * *[-,-]$ \\
\hline Averill $(2016)^{\mathrm{f}}$ & 851 & $M=412$ days & $.33 * *$ & $-[-,-]$ & $.39 * *$ & $-[-,-]$ & $-.33 * *$ & $-[-,-]$ & $.39 * *$ & $-[-,-]$ \\
\hline \multicolumn{11}{|c|}{ General recidivism (excluding supervision violations) } \\
\hline Hanby $(2013)^{b}$ & 1775 & 2 years & - & $.61[.59, .64]$ & - & $.66[.63, .68]$ & - & $.61[.58, .64]$ & - & $.65[.62, .68]$ \\
\hline Hanby $(2013)^{c}$ & 3372 & 2 years & - & $.62[.60, .64]$ & - & $.67[.65, .69]$ & - & $.62[.60, .64]$ & - & $.66[.64, .68]$ \\
\hline Chadwick (2014) & 391 & $M=250$ days & - & $.52[.42, .69]$ & - & $.53[.43, .63]$ & - & $.56[.47, .66]$ & - & $.53[.43, .63]$ \\
\hline Yesberg \& Polaschek $(2015)^{\mathrm{e}}$ & 299 & 6 months & - & $.61^{* *}[-,-]$ & - & $.57 *[-,-]$ & - & $.60 * *[-,-]$ & - & $.62 * *[-,-]$ \\
\hline Ferguson $(2015)^{\mathrm{g}}$ & 122 & $M=809$ days & - & $.74[.64, .84]$ & - & $.68[.56, .79]$ & - & $.65[.54, .76]$ & - & $.74[.63, .84]$ \\
\hline Ferguson $(2015)^{\mathrm{h}}$ & 122 & $M=812$ days & - & $.64[.53, .76]$ & - & $.63[.51, .75]$ & - & $.59[.47, .70]$ & - & $.65[.54, .76]$ \\
\hline Averill $(2016)^{\mathrm{f}}$ & 851 & $M=412$ days & $.20 * *$ & $-[-,-]$ & $.24 * *$ & $-[-,-]$ & $-.21 * *$ & $-[-,-]$ & $.24 * *$ & $-[-,-]$ \\
\hline Serin et al. $(2016)^{\mathrm{i}}$ & 563 & 3 months & - & $.67[.59, .75]$ & - & $.68[.58, .77]$ & - & $.67[.59, .75]$ & - & $.70[.61, .78]$ \\
\hline Muirhead $(2016)^{j}$ & 369 & $M=973$ days & - & $.70[.64, .76]$ & - & $.69[.63, .74]$ & - & $.68[.62, .74]$ & - & $.73[.67, .78]$ \\
\hline \multicolumn{11}{|l|}{ Sexual recidivism } \\
\hline Smeth $(2013)^{\mathrm{k}}$ & 193 & $M=22$ months & $.01_{\mathrm{ns}}$ & $.51[.26, .75]$ & $.05_{\mathrm{ns}}$ & $.53[.30, .75]$ & $.01_{\mathrm{ns}}$ & $.46[.21, .71]$ & $.02_{\mathrm{ns}}$ & $.46[.22, .70]$ \\
\hline Averill $(2016)^{\mathrm{f}}$ & 851 & $M=412$ days & $.02 \mathrm{~ns}$ & $-[-,-]$ & $.07 *$ & $-[-,-]$ & $-.06_{\mathrm{ns}}$ & $-[-,-]$ & $.06_{\mathrm{ns}}$ & $-[-,-]$ \\
\hline \multicolumn{11}{|l|}{ Violent recidivism } \\
\hline Yesberg \& Polaschek $(2015)^{\mathrm{e}}$ & 299 & 6 months & - & $.58_{\mathrm{ns}}[-,-]$ & - & $.55_{\mathrm{ns}}[-,-]$ & - & $.61_{\mathrm{ns}}[-,-]$ & - & $.60_{\mathrm{ns}}[-,-]$ \\
\hline Averill $(2016)^{\mathrm{f}}$ & 851 & $M=412$ days & $.18^{* *}$ & $\begin{array}{l}-[-,-] \\
-[-,]\end{array}$ & $.26 * *$ & $\begin{array}{l}-[-,-] \\
-[\end{array}$ & $-.17 * *$ & $\begin{array}{l}-[-,-] \\
-[\end{array}$ & $.23 * *$ & $\begin{array}{l}-[-,-] \\
-\left[\begin{array}{l}0 \\
{[}\end{array}\right]\end{array}$ \\
\hline Muirhead (2016) & 397 & $M=973$ days & - & $.61[.54, .68]$ & - & $.68[.61, .75]$ & - & $.62[.55, .68]$ & - & $.66[.59, .73]$ \\
\hline \multicolumn{11}{|l|}{ Supervision violations } \\
\hline Smeth $(2013)^{\mathrm{k}}$ & 193 & $M=22$ months & $.33 * *$ & $.69[.62, .78]$ & $.27 * *$ & $.65[.57, .74]$ & $-.18^{*}$ & $.61[.52, .69]$ & $.31 * *$ & $.68[.60, .76]$ \\
\hline Chadwick (2014) & 391 & $M=250$ days & - & $.61[.56, .67]$ & - & $.59[.53, .65]$ & - & $.57[.51, .63]$ & - & $.61[.56, .67]$ \\
\hline Yesberg \& Polaschek $(2015)^{\mathrm{e}}$ & 299 & 6 months & - & $.53_{\mathrm{ns}}[-,--]$ & - & $.53_{\mathrm{ns}}[-,-]$ & - & $.58 *[-,-]$ & - & $.55_{\mathrm{ns}}[-,-]$ \\
\hline Ferguson $(2015)^{\mathrm{g}}$ & 101 & $M=270$ days & - & $.72[.62, .81]$ & - & $.70[.59, .81]$ & - & $.65[.55, .76]$ & - & $.71[.60, .81]$ \\
\hline Ferguson $(2015)^{\mathrm{h}}$ & 101 & $M=266$ days & - & $.68[.52, .85]$ & - & $.77[.63, .91]$ & - & $.59[.46, .71]$ & - & $.74[.61, .87]$ \\
\hline Averill $(2016)^{\mathrm{f}}$ & 851 & $M=412$ days & $.31 * *$ & $-[-,-]$ & $.37 * *$ & $-[-,-]$ & $-.31 * *$ & $-[-,-]$ & $.37 * *$ & $-[-,-]$ \\
\hline
\end{tabular}


Serin et al. (2016) ${ }_{\text {any }}^{\mathrm{i}}$

Note. DRAOR = Dynamic Risk Assessment for Offender Re-Entry; $r=$ point-biserial correlation. AUC $=$ area under the curve statistic; $\mathrm{CI}=$ confidence interval; ns = nonsignificant. Adapted from Lloyd (2015) and Pardoel (2018).

${ }^{\mathrm{a}}$ Mean follow-up times are reported for studies with variable follow-up periods; all other follow-ups were fixed. ${ }^{\mathrm{b}}$ Adult male and female general offenders in New Zealand (Māori offenders only; Hanby 2013). ${ }^{\mathrm{c}}$ Adult male and female general offenders in New Zealand (overall sample; Hanby 2013). ${ }^{\mathrm{d} A d u l t}$ male and female general offenders in Iowa (Chadwick, 2014). ${ }^{\mathrm{e}}$ Adult male high-risk offenders in New Zealand (Yesberg \& Polaschek, 2015). ${ }^{\mathrm{f}}$ Adult male sex offenders in New Zealand (Averill, 2016). ${ }^{\mathrm{g}}$ Adolescent male general offenders in New Zealand; AUCs reported in this summary table are based on proximal DRAOR scores (Ferguson, 2015). ${ }^{\mathrm{h}}$ Adult male general offenders in New Zealand; AUCs reported in this summary table are based on proximal DRAOR scores (Ferguson, 2015). ${ }^{\mathrm{i}}$ Adult male and female general offenders in Iowa (Serin et al., 2016). ${ }^{\mathrm{j}}$ Adolescent male and female general offenders in New Zealand; AUCs reported in this summary table are based on proximal DRAOR scores (Muirhead, 2016). ${ }^{\text {k} A d u l t ~ m a l e ~ s e x ~ o f f e n d e r s ~ i n ~ I o w a ~(S m e t h, ~ 2013) . ~}$

$* p<.05 . * * p<.01$ 
Incremental predictive validity. This type of validity investigates whether a particular scale or variable increases the predictive ability of other scales or variables. In this case, the DRAOR Total and the Stable, Acute, and Protective subscales were examined to see if they predict community outcomes over and above existing risk scales. In a study on 3,498 New Zealand parolees, the DRAOR Total improved the ability of the RoC*RoI-a static risk scaleto predict any reoffence and criminal reoffence for the overall sample, as well as for Māori offenders (Hanby, 2013). An additional study found that the DRAOR Total added predictive power to the $\mathrm{RoC}^{*} \mathrm{RoI}$ for women when predicting general recidivism, but not for men (Yesberg et al., 2015). The Acute subscale also predicted general recidivism for women over and above the RoC*RoI in Yesberg and colleagues' (2015) study. Note that both studies evaluated incremental validity using Cox regression.

Yesberg and Polaschek (2015) examined the incremental predictive validity of the DRAOR among 299 high-risk offenders using hierarchical logistic regression. The DRAOR Total improved the prediction of reconvictions when added to the RoC*RoI, VRS (a static and dynamic scale), and RPFA-R (a dynamic measure of preparedness for release); however, the VRS and RPFA-R did not make significant unique contributions to the prediction of reconvictions when the DRAOR Total was added to the model. Notably, the DRAOR Total did not improve the prediction of reimprisonment when added to the VRS Dynamic scale. ${ }^{11}$ Yesberg and Polaschek also assessed the ability of their revised Stable subscale ${ }^{12}$ to predict reconvictions over and above the other scales. This new Stable domain improved the prediction of

\footnotetext{
${ }^{11}$ The VRS Static scale, RoC*RoI, and RPFA-R did not predict reimprisonment on their own so they were not included in this analysis.

${ }^{12}$ Preliminary analyses showed that the new DRAOR Stable was the only subscale to significantly predict recidivism, and reconviction was the only outcome it predicted. Therefore, hierarchical logistic regression analyses were limited to these variables. The new Stable subscale includes opportunity/access to victims and employment.
} 
reconvictions when added to the RoC*RoI, VRS, and RPFA-R, but the contributions of the VRS and RPFA-R were no longer significant.

The DRAOR has also demonstrated incremental predictive validity in two samples of sex offenders. Smeth (2013) found that the DRAOR Total, as well as the Stable, Acute, and Protective subscales improved the prediction of time to parole violations when added independently to the Static-99R in a series of Cox regression survival analyses, with the Static99 only remaining significant with the Protective subscale $(N=193)$. The three subscales (Stable, Acute, and Protective) were also added to the Static99R concurrently to see if any uniquely predicted time to parole violations when controlling for the contributions of static risk and the other two subscales. In this analysis, only the Stable subscale uniquely predicted recidivism. The author did not examine whether DRAOR improved sexual recidivism prediction beyond static risk because neither the overall scale nor the subscales predicted time to sexual recidivism in univariate logistic regression analyses.

In the second study on 851 sex offenders, Averill (2016) used Cox regression survival analyses to explore the incremental predictive validity of the DRAOR over and above static risk. For time to sexual recidivism, neither the DRAOR Total, nor the subscales independently added predictive power to the ASRS. The three subscales (Stable, Acute, and Protective) did not significantly predict time to sexual recidivism when added simultaneously to the model either. Notably, the Stable subscale made a unique contribution to the model in the opposite direction expected (i.e., higher Stable scores accounted for lower sexual recidivism). In terms of time to violent and general recidivism, the DRAOR Total and the Stable, Acute, and Protective subscales independently improved the predictive accuracy of the RoC*RoI. Simultaneously adding the three DRAOR subscales to the RoC*RoI also resulted in improved prediction for time to violent 
and general reoffence. The Acute subscale uniquely predicted time to violent recidivism, and the Protective subscale uniquely predicted time to general recidivism when controlling for the contribution of the RoC*RoI and the other subscales.

The DRAOR appears to add incremental predictive validity to a variety of risk instruments for numerous recidivism outcomes. For example, the DRAOR Total improved the prediction of general and violent recidivism, reconvictions, and parole violations beyond static risk, as well as the prediction of reconvictions beyond other dynamic risk measures. A revised version of the Stable subscale, which saw the addition of two items, ${ }^{13}$ improved the prediction of reconvictions when added to static and dynamic risk scales. The DRAOR Stable, Acute, and Protective subscales improved the prediction of parole violations when added independently to a static risk instrument. Further, the Stable subscale predicted parole violations over static risk and the other DRAOR subscales; the Acute subscale predicted general and violent recidivism over static risk, other dynamic risk measures, and the other DRAOR subscales; and the Protective subscale predicted general and violent recidivism over static risk, as well as the other DRAOR subscales. Despite these generally positive findings, there is little evidence to suggest that the DRAOR predicts sexual recidivism risk beyond static risk. The above research supports integrating the DRAOR into current risk assessment practices to improve our ability to predict recidivism and refine case management strategies.

Inter-rater reliability. The consistency in risk ratings for the same offenders across evaluators is an important aspect of scale validation. This is especially true for instruments that include dynamic risk factors, which involve a degree of interpretation, such as gauging offenders' attitudes or values (Latessa \& Lovins, 2010). For example, previous research found

\footnotetext{
${ }^{13}$ Recall that Yesberg and Polaschek's (2015) revised Stable subscale includes opportunity/access to victims and employment, which are part of the Acute subscale in the original DRAOR.
} 
the lowest inter-rater reliability estimates for items reflecting a higher degree of subjectivity and psychological-relevance, as summarized by Lloyd (2015, pp. 134). Poor inter-rater reliability may be due to ambiguous theoretical underpinnings of a tool, unclear transfer of knowledge to evaluators, or the scale items being inapplicable to specific group of offenders (Lloyd, 2015). Examining the inter-rater reliability of a risk tool can therefore help determine whether raters understand the theoretical basis of items and can recognize these factors in offenders (Lloyd, 2015).

Despite dynamic risk scales being more difficult to score than static, fact-based risk scales due to their subjective nature, previous research shows that dynamic risk items can indeed be reliably measured. Desmarais, Nicholls, Wilson, and Brink (2012) found excellent inter-rater reliability for Strength $\left(\mathrm{ICC}^{14}=.93\right)$ and Vulnerability $(\mathrm{ICC}=.95)$ subscales of the START, a dynamic SPJ instrument, for example. Further, inter-rater reliability of the dynamic subscale of the Violence Risk Scale: Sexual Offender Version—an actuarial instrument comprised of 17 dynamic and seven static risk factors - was excellent for both pre- and post-treatment assessments (ICC $=.90$ and .92 , respectively; Beggs \& Grace, 2010). Earlier research also found excellent inter-rater reliability for the Stable-2000, a 16-item dynamic risk measure (ICC = .89; Hanson et al., 2007).

The above findings show promise for evaluators' ability to accurately measure subjective items, however, a shortcoming and common criticism of risk assessment research is that it fails to consistently evaluate inter-rater reliability. Highlighting this issue, Desmarais and Singh (2013) reviewed 53 validation studies on general risk assessment tools used with adult offenders in the U.S., and found that only two of these studies examined inter-rater reliability. The paucity

\footnotetext{
${ }^{14}$ Intra-class correlation coefficients (ICC) can range from 0 to 1 , and are categorized as either poor $(<.40)$, fair $(.40$ $.50)$, good (.60-.74) or excellent (>.75; Cicchetti, 2001).
} 
of inter-rater reliability research relative to research on other psychometric properties is implicit to the nature of correctional practice. Namely, it is uncommon for two officers to supervise the same offender, making inter-rater reliability less feasible to measure than other psychometric properties (Morgan, Kroner, Mills, Serna, \& McDonald, 2013). One way around this practicality issue is to assess inter-rater reliability in lab settings using vignettes or by comparing officers' field ratings to researchers' file reviews (Lloyd, 2015). An important limitation of these methods, however, is their low ecological validity (i.e., potentially limited generalizability to real-life settings; Vincent, Guy, Fusco, \& Gershenson, 2012). Nonetheless, in the absence of more ecologically valid methods of measuring inter-rater reliability (e.g., risk assessment ratings from clinicians in opposing sides of a criminal trial; Boccaccini, Turner, \& Murrie, 2008; Murrie, Boccaccini, Johnson, \& Janke, 2008), vignettes or comparisons of officers' and researchers' risk evaluations can provide important information on scale reliability.

A limitation of the DRAOR is that there is currently no research on its inter-rater reliability. However, this scale property has been measured using the institutional version of the DRAOR, the Structured Dynamic Assessment Case-Management-21 (SDAC-21; see Appendix C; Serin \& Wilson, 2013, 2017). The SDAC-21 is a 21-item clinical rating scale, developed after the successful implementation of the DRAOR in New Zealand. It was modelled after the DRAOR and strongly aligns with the relevant subscales. Specifically, it evaluates Stable risk, Responsivity, and Protective factors. The Stable and Protective domains are largely the same across the institutional and community versions of the scale, with the Responsivity domain accounting for much of the variation in the two versions. Responsivity factors relate to the competencies, motivation, and learning difficulties that act as barriers to change and successful rehabilitation. 
Sixteen of the SDAC-21 items were sourced from the DRAOR, including gang (peer) associations, attitudes toward authority, impulse control, problem solving, sense of entitlement, attachment with others, substance abuse, negative mood, anger/hostility, responsive to advice, prosocial identity, high expectations, costs/benefits, social support, social control, and employability (employment). Given that the SDAC-21 was modelled after the DRAOR and has overlapping items, research on the inter-rater reliability of this scale can provide insight into the reliability of DRAOR when used by multiple evaluators to assess the same offenders.

Recently, Smeth (2018) reported on the inter-rater reliability of the SDAC-21 using twenty randomly selected audiotaped interviews between officers and clients (see Table 2). A state-level trainer independently reviewed offenders' files, listened to the audiotapes, and rated the offender on the SDAC-21. Inter-rater reliability was determined by comparing these ratings with the officers' ratings. Inter-rater reliability estimates for SDAC-21 items that overlap with DRAOR Stable items ${ }^{15}$ ranged from good to excellent (ICCs $=.62$ to .96). SDAC-21 items represented in the DRAOR Acute domain were generally high, ranging from good to excellent for anger/hostility, negative mood, and employability items (employment in the DRAOR; ICCs $=.64$ to .82$)$, with only the substance abuse item producing fair inter-rater reliability $(\mathrm{ICC}=.54)$. Lastly, SDAC-21 Protective items that overlap with DRAOR Protective items ${ }^{16}$ produced good to excellent levels of inter-rater reliability (ICCs $=.67$ to .80$)$. While these results cannot be used as direct evidence for the inter-rater reliability of the DRAOR, they serve as initial support for the use of the scale to reliably measure dynamic risk and protective factors.

\footnotetext{
${ }^{15}$ All DRAOR Stable items are represented in the SDAC-21 Stable domain: gang (peer) associations, attitude toward authority, impulse control, problem solving, sense of entitlement, and attachment with others.

${ }^{16}$ All DRAOR Protective items are represented in the SDAC-21 Protective domain: responsive to advice, prosocial identity, high expectations, costs/benefits, social support, and social control.
} 
Table 2

Inter-Rater Reliability (ICCS) for the SDAC-21

\begin{tabular}{ll}
\hline SDAC-21 item & ICC \\
\hline Stable & \\
Gang Associations & .96 \\
Attitude Toward Authority & $\mathbf{. 8 6}$ \\
Impulse Control & $\mathbf{. 6 2}$ \\
Problem Solving & $\mathbf{. 6 6}$ \\
Sense of Entitlement & .74 \\
Attachment with Others & .85 \\
Substance Abuse & .54 \\
Responsivity & \\
Health Problems & .97 \\
Conduct Issues & .87 \\
Negative Mood & .82 \\
Unresponsive Rehabilitation & .89 \\
Anger/Hostility & $\mathbf{. 6 4}$ \\
Offence Mirroring Behaviours & .87 \\
Learning Difficulties & .85 \\
Protective & \\
Responsive to Advice & .70 \\
Prosocial Identity & .75 \\
High Expectations &. $\mathbf{8 0}$ \\
Costs/Benefits & .73 \\
Social Support & .72 \\
Social Control & .67 \\
Employability & .74 \\
Average ICC & .77 \\
\hline
\end{tabular}

Note. Overlapping SDAC-21 and DRAOR items are bolded. SDAC-21 = Structured Dynamic

Assessment Case-Management-21; ICC = intra-class correlation coefficient.

${ }^{\mathrm{a} A v e r a g e ~ I C C ~ a c r o s s ~} 20$ assessments.

Implementation issues. In addition to the unknown inter-rater reliability of the DRAOR, there is limited research on whether the DRAOR has been implemented with fidelity. This can be measured by assessing officer training and the amount of experience raters have with a given tool (Flores, Lowenkamp, Holsinger, \& Latessa, 2006). For example, the Level of Service Inventory-Revised (LSI-R) significantly predicted recidivism for trained raters $(r=.21, p<.01)$, 
but not untrained raters $(r=.08, \mathrm{~ns})$, suggesting that untrained professionals using the LSI-R cannot accurately predict future criminal behaviour (Flores et al., 2006). This finding calls into question the practice of basing case plans and classification decisions on untrained professionals' LSI-R ratings (Flores et al., 2006). Flores and colleagues (2006) also found that the LSI-R produced more accurate assessments of recidivism risk when scored by raters with more experience using the instrument $(r=.25, p<.01$ for those with more than three years of experience; $r=.14, p<.01$ for those with less than three years of experience). Moreover, earlier research by Bonta, Bogue, Crowley, and Motiuk (2001) demonstrated that ongoing staff training reduced the number of risk prediction errors.

It is clear that poor implementation integrity has the potential to degrade the predictive validity of an instrument. However, risk assessment research often does not take this into account and assumes that risk tools have been implemented with fidelity (Flores et al., 2006). This is a second limitation of research on the DRAOR, with the effects of training on predictive validity only examined in one study to date. Namely, Chadwick (2014) found that the DRAOR Total and three domains significantly predicted general recidivism for formally trained officers (AUCs ranged from .62 to .68), but not informally trained officers (AUCs ranged from .52 to .55). These results raise concerns about the utility of untrained officers' DRAOR assessments in case planning and risk prediction. An important strength of the current research, however, is that all officers received formal training from the scale developer. Further, as demonstrated by Flores et al. (2006) and Chadwick (2014), differences in training reduces_-rather than artificially inflates-predictive validity (Lloyd, 2015).

Another issue related to the implementation of the DRAOR is the degree to which officers effectively supervise offenders on their caseloads. Effective supervision adheres to core 
correction practice (CCP), which is based on empirically validated strategies for intervention (Andrews \& Kiessling, 1980). Its main goal is to foster positive behavioural change in offenders through (1) the effective use of authority (correctional staff should be firm but fair in their approach), (2) anticriminal modeling and reinforcement (officers should encourage prosocial attitudes and behaviours through positive and negative reinforcement), (3) problem solving (officers should teach offenders how to overcome obstacles in prosocial ways), (4) use of community resources (officers should provide the offender with appropriate services), and (5) interpersonal relationships between staff and offenders (i.e., correctional services are the most effective when there is mutual respect and liking between officers and offenders; Dowden \& Andrews, 2004).

The DRAOR complements CCP by identifying changes in client needs that warrant intervention. This is done both within a session and across sessions over time. As the DRAOR serves as a tool to assess risk, as well as a tool for supporting offender intervention, it is important to determine how differences in supervision efficacy influence the predictive validity of the scale. For example, the DRAOR largely requires self-report information from offenders (except some items, like substance abuse, which can be scored based on urinalysis or drug tests), thus the accuracy of scores depends, in part, on officers' ability to obtain the necessary information from offenders. Offenders' self-disclosure of pertinent information may be associated with the quality of their relationship with their supervising officer, a key element of CCP. For example, in a study on patients' self-disclosure in psychotherapy, working alliance (i.e., the collaborative and affective relationship between a therapist and client; Martin, Garske, \& Davis, 2000) significantly predicted client-openness, such that clients who reported better working alliances were also more open in their disclosures (Pattee \& Farber, 2008). Research on 
working alliance in correctional settings shows that officers' characteristics (e.g., caring, fair, trusting, involved), supervision style (e.g., authoritative versus authoritarian), and relationship and rapport building skills affect alliance quality (Bonta et al., 2010; Kennealy, Skeem, Eno Louden, \& Manchak, 2012; Skeem, Louden, Polaschek, \& Camp, 2007).

In addition to influencing offender openness, the working alliance is a critical aspect of the change process; a strong alliance between the therapist and client leads to more successful treatment outcomes (Bordin, 1979). In correctional settings, a good working alliance between officers and offenders has led to fewer re-arrests and probation revocations (Bonta et al., 2010; Kennealy et al., 2012; Skeem et al., 2007). Adherence to other CCP elements may also influence offenders' success in the community. The extent to which officers teach offenders how to overcome obstacles in prosocial ways and use anticriminal modeling and reinforcement strategies could influence offenders' recidivism risk, for example.

The quality of supervision can therefore influence the predictive validity of the DRAOR such that effective officers will (a) rate DRAOR items more accurately by evoking more open responses from offenders, increasing the potential association between DRAOR scores and recidivism, and (b) better manage offender risk by adhering to $\mathrm{CCP}$, reducing the potential association between high DRAOR scores and recidivism (Lloyd, 2015). Thus, the effects of supervision quality on the predictive accuracy of the DRAOR can be explored by measuring officers' characteristics and skills as they relate to CCP. There is currently no research in this area, however, which represents an additional weakness of the current and previous DRAOR works.

Summary. Research on the DRAOR has focused on its factor structure, internal consistency, convergent and divergent validity with other validated risk measures, and predictive 
validity (both independently and beyond static risk). An important area of research that requires attention is the implementation of the DRAOR. Officers likely differ in the extent to which they implement the scale with fidelity. For example, officers who understand the theoretical basis of items and can recognize these factors in offenders will score items more accurately, affecting inter-rater reliability. Further, officers' characteristics and skills can (1) potentially improve the accuracy of DRAOR ratings by eliciting more open self-disclosures from offenders and (2) facilitate prosocial attitude and behaviour changes among offenders, possibly reducing the association between high DRAOR scores and recidivism. Given the uncertainty of how the DRAOR has been implemented in practice, conclusions from previous research and the current study may be limited. Issues regarding implementation fidelity, however, are more likely to minimize the predictive effects of the DRAOR than artificially inflate findings (Lloyd, 2015).

\section{DRAOR With Intimate Partner Violence Offenders}

The DRAOR has been used to predict recidivism among males and females, adults and adolescents, Māori and non-Māori offenders, as well as various offender groups, such as general, violent, nonviolent, and sex offenders. One population the instrument has not be validated on, however, is IPV offenders. Both the prevalence of IPV and the public health costs associated with this type of violence highlight the importance of accurate risk assessment among IPV offenders.

Existing meta-analyses on the accuracy of IPV risk assessments (Helmus \& Bourgon, 2011; Messing \& Thaller, 2013) find lower effect sizes than reported for either general offending (Bonta, Blais, \& Wilson, 2014; Campbell et al., 2009) or specific offence subtypes such as sex offending (Hanson \& Morton-Bourgon, 2009) and violent offending (Yang et al., 2010). The most promising IPV risk scales appear to be the ODARA and the SARA, which, in recent 
research, yielded AUCs ranging from .66 to .72 (moderate to large effects) and .62 to .74 (small to large effects), respectively, for IPV recidivism (Jung \& Buro, 2017; Messing \& Thaller, 2013; Olver \& Jung, 2017).

Lower effect sizes for IPV risk instruments may, in part, be due to a less developed effort to identify the strongest predictors of IPV (Z. Hilton, personal communication, May 17, 2017). Given this, there is no current 'best set' of IPV predictors; however, numerous variables have been identified in the literature as being significantly associated with IPV recidivism, one of which is substance abuse. In a critical review of 62 studies published between January 1990 and September 2003, Bennett Cattaneo and Goodman (2005) identified common risk factors for repeat male-perpetrated IPV against women. Alcohol and/or drug abuse was the most commonly identified dynamic risk predictor, reported as being significantly associated with reabuse in eight of the 16 studies assessing this variable (six positively and two negatively associated; Bennett, Goodman, \& Dutton, 2000; Bennett Cattaneo \& Goodman, 2003; Chaudhuri \& Daly, 1992; Hamberger \& Hastings, 1990; McCarroll, 2000; Meloy, Cowett, Parker, Hofland, \& Friedland, 1997; Miller \& Krull, 1997; Snow Jones \& Gondolf, 2001). Authors of a recent validation study on a domestic abuse risk assessment tool in the U.K. (Domestic Abuse, Stalking and harassment and Honour-based violence; DASH) also reported that problematic alcohol use was significantly positively related to repeat general and violent domestic abuse (note that offenders' gender was not specified; Almond, McManus, Brian, \& Merrington, 2017). Substance abuse has further been identified as a significant predictor of male- and female-perpetrated IPV onset and recidivism in various other studies (Brem et al., 2018; Fals-Stewart, 2003; Gordon \& Moriarty, 2003; Murphy, Morrel, Elliott, \& Neavins, 2003; Shepard, 1992; Tollefson \& Gross, 2006; Whitaker, Le, \& Niolon, 2010). 
In their review of factors predicting male-perpetrated wife assault, Hilton and Harris (2005) reported that, despite separation and/or no-contact orders, many wife assaulters recidivate against the same victim. Almond and colleagues (2017) also found that women were more likely to be nonviolently reassaulted by their abusers if they "constantly texted, called, followed, stalked, or harassed" them (p. 68). Further, in their critical review of key studies, Bennett Cattaneo and Goodman (2005) identified "living together" as a predictor of IPV recidivism among male offenders in five of nine studies assessing this variable (three positively and two negatively associated; Ford \& Regoli, 1992; Gondolf, 1997; Harrell \& Smith, 1996; Miller \& Krull, 1997; Wooldredge \& Thistlewaite, 2002). Living together has also been reported as a significant predictor of persistent physical partner aggression among male and female perpetrators in a more recent study that involved adolescent and adult couples (Whitaker et al., 2010). These findings generally suggest that IPV offenders who have access to their previous or current intimate partners are more likely to recidivate.

An additional risk factor for repeat wife assault identified in Hilton and Harris’ (2005) review is marital conflict. Earlier research found this variable to be especially useful for predicting the onset of wife assault among newlyweds (Leonard \& Senchak, 1996). Marital conflict has also been linked to both the occurrence and severity of violence among male wife assaulters (Aldarondo \& Sugarman, 1996). Further, recent research provides a detailed exploration of relationship problems reported by male IPV offenders and found that problem severity was significantly associated with physical and emotional IPV perpetration (LaMotte, Meis, Winters, Barry, \& Murphy, 2018). Core problems included communication/money management, substance use, sexual difficulties, cultural differences, mistrust/jealousy, togetherness, and shared friends/activities (LaMotte et al. 2018). 
Unemployment has also been linked to IPV recidivism. For example, four of the 16 studies in Bennett Cattaneo and Goodman's (2005) review that assessed this variable found that male offenders who were unemployed or employed only part-time had higher rates of IPV reoffending (Babcock \& Steiner, 1999; Chaudhuri \& Daly, 1992; Feder \& Dugan, 2002; Taylor, Davis, \& Maxwell, 2001). Male unemployment was also predictive of repeat male-to-female IPV in a five-year longitudinal study on a U.S. sample of adult couples (Caetano, McGrath, Ramisetty-Mikler, \& Field, 2005). Additionally, unemployment has been implicated in the persistence and severity of IPV in a critical review of the literature. Edwards (2015) argues that higher rates of unemployment among rural-based perpetrators may be associated with these individuals committing more chronic and severe IPV than urban-based perpetrators (note that offenders' gender was not specified for all studies reviewed, but several involved males only). Another variable that is empirically related to repeat IPV offending - though perhaps not as commonly examined as substance abuse, access to victims, marital conflict, and unemployment—is negative affect/mood. Early research found that "batterer depression" and "husband's global negative affect" were significantly related to IPV recidivism (Jacobson, Gottman, Gortner, Berns, \& Shortt, 1996; Palmer, Brown, \& Barrera, 1992). More recently, Shorey and colleagues found that a composite negative affect score, as well as the individual negative affect items angry, frustrated, hostile, irritated, mad, and upset predicted psychological intimate partner aggression perpetration among male undergraduate university students (Shorey, McNulty, Moore, \& Stuart, 2015). Individual negative affect items anxious, frustrated, hostile, irritated, mad, and sad — but not the composite negative affect score—predicted physical intimate partner aggression perpetration in this same study (Shorey et al., 2015). Emotion regulation also demonstrated a moderating effect between negative affect and physical intimate partner 
aggression perpetration: negative affect led to higher odds of physical violence when emotion regulation difficulties were high, but not low (Shorey et al., 2015). A meta-analytic review provides additional evidence for the relationship between negative emotions and IPV perpetration among males and females, with effect sizes between the two variables generally moderate in strength (Birkley \& Eckhardt, 2015).

Anger and hostility have both demonstrated utility in predicting IPV. As mentioned above, Shorey and colleagues (2015) found anger and hostility to predict psychological and physical intimate partner aggression perpetration among male undergraduate university students. Earlier research by Shorey and colleagues examined the incremental validity of anger over and above impulsivity when predicting female-perpetrated psychological and physical aggression. Regression analyses revealed that anger significantly predicted IPV over and above impulsivity, and that impulsivity was no longer significant when anger was added to the model (Shorey, Brasfield, Febres, \& Stuart, 2011). Anger was also a significant predictor of aggressive verbalizations among married violent men, but not married nonviolent men (Eckhardt, 2007), Moreover, anger and hostility were moderately associated with male and female IPV perpetration in two meta-analytic reviews (Birkley \& Eckhardt, 2015; Norlander \& Eckhardt, 2005). Those who perpetrated more severe violence towards their intimate partners reported higher levels of anger and hostility than those who perpetrated less severe violence towards their intimate partners, indicating that anger and hostility are implicated in both the occurrence and severity of IPV (Birkley \& Eckhardt, 2015; Norlander \& Eckhardt, 2005).

Impulsivity is identified as a key feature of antisocial personality pattern/disorder, which is one of the Big Four predictors of criminal behaviour (American Psychiatric Association, 2013; Andrews \& Bonta, 2010). A recent study found that antisocial personality disorder significantly 
and positively predicted psychological aggression and physical assault perpetration in men arrested for domestic violence (Brem et al., 2018). Other research examined the interaction between perpetrator impulsivity_including negative and positive urgency, which refer to the tendencies to act impulsively in response to either negative or positive affect - and victim alcohol use on male- and female-perpetrated IPV in heterosexual couples (Leone, Crane, Parrott, \& Eckhardt, 2016). Leone and colleagues (2016) reported significant positive associations between perpetrator urgency (both negative and positive) and physical IPV for those with partners who displayed problematic and non-problematic drinking, though these relationships were stronger among those with non-problematic drinking partners. Impulsivity also predicted psychological and physical aggression in a female sample of IPV offenders (Shorey et al., 2011).

These IPV risk factors map onto several of the DRAOR items, including substance abuse (alcohol and/or drug abuse), opportunity/access to victims (stalking/harassing behaviour, the offender and victim living together), attachment with others and interpersonal relationships (marital conflict/relationship problems), employment (the offender being unemployed or employed part-time only), negative mood (the offender being depressed or having a negative affect), anger/hostility, and impulse control (negative/positive urgency, antisocial personality disorder, impulsivity). Attachment with others and impulse control are in the Stable domain and the other items are in the Acute domain. These items were selected for inclusion in the DRAOR given their empirical relationship with increased risk of general recidivism and their sensitivity to fluctuations in risk.

Notably, addictions and substance abuse are key correlates of crime, with the maintenance of sobriety and avoidance of drugs playing an important role in the crimedesistance process (Andrews et al., 2006; Andrews \& Bonta, 2010; Bennett, Holloway, \& 
Farrington, 2008). In the DRAOR, problematic substance use could involve a functional link to reoffending (e.g., disinhibition or to gain money to continue substance use) or acting as a barrier to engagement in prosocial activities and/or support associated with crime desistance (Serin, $2007,2015,2017)$. This item is rated as a current risk based on recent behaviour and significant patterns of substance abuse (Serin, 2007, 2015, 2017).

Opportunity or access to victims also increases the likelihood of reoffending; offenders with a preferred victim or pattern of victim selection are more likely to recidivate if they have access to said victim-type (Hanson \& Harris, 1998). A domestic abuser in a relationship or living with his partner, for example, would be rated as having opportunity/access to his victims in the DRAOR (Serin, 2007, 2015, 2017).

Family/marital problems is one of the Central Eight risk factors for criminal behaviour (Andrews \& Bonta, 2010) and has been linked to IPV (Hilton \& Harris, 2005; LaMotte et al. 2018). This factor is reflected in the DRAOR items attachment with others (Stable) and interpersonal relationships (Acute). Supervision officers classify offenders' attachment with others as problematic if they have a characteristic, ongoing lack of concern for others (either due to poor perspective taking or shallow emotional experiences) that results in social disconnection or interpersonal attachments issues. Offenders are rated as having poor interpersonal relationships if their intimate partnerships involve antagonism, victimization, a breakdown, disconnection, or social pressure to engage in criminal activity, for instance.

Employment/job satisfaction is another important factor that fosters crime desistance (Laub \& Sampson, 2001; Tripodi, Kim, \& Bender, 2010; Uggen, 1999, 2000). DRAOR evaluators identify the presence/absence of employment, as well as job preference/longevity, 
type of employment, the level of income it will bring, and the skills of the offender in maintaining employment when scoring this item (Serin, 2007, 2015, 2017).

Negative mood — often operationalized as being depressed or anxious — has been identified as a precursor or antecedent to criminal behaviour (Chui \& Chan, 2012; Hanson \& Harris, 2000; Hudson, Ward, \& McCormack; 1999; Turner, 2001; Ward, Hudson, \& Marshall; 1995). When scoring this DRAOR item, evaluators assess whether the offender is sad, hopeless, uneasy, fearful, worried, tense, jumpy, restless, or has poor concentration (Serin, 2007, 2015, 2017).

Anger (emotional volatility) and hostility (antagonism towards others) have been related to various types of violent crime, including homicide, violence between intimate partners, child abuse, and sex offending (Baron, 2009; Fiqia, Lang, Plutchik, \& Holden, 1987; Howells, 2004; Nyamathi et al., 2017). Individuals who experience anger and hostility are noted in the DRAOR as seeming to engage with the world in an unfriendly way, being sensitive to perceived rejection, cynically judging others as negatively motivated, being callous, rude, easily irritated and frustrated, and often making statements relating to retribution (Serin, 2007, 2015, 2017). Evaluators therefore take these characteristics and behaviours into consideration when scoring this item.

Lastly, impulse control problems are among the primary personality correlates of crime and are implicated in the development of stable, long-term, serious antisocial behaviour (Moffit, 1993; White et al., 1994). There is meta-analytic evidence for low self-control as one of the strongest predictors of crime (Pratt \& Cullen, 2000) and impulsivity is a defining feature of antisocial personality pattern, one of the Big Four predictors of criminal behaviour (Andrews \& Bonta, 2010). DRAOR evaluators assess offenders' spontaneity and consideration of potential 
consequences of their actions when scoring this item and note the following as signs of having impulse control problems: frequent job changes, frequent relationship changes, boredom, poly substance abuse, binge drinking, and risk-taking behaviours (Serin, 2007, 2015, 2017).

Most of the research on the relationship between protective factors and adult IPV concerns victimization rather than perpetration (e.g., Boyle, Georgiades, Cullen, \& Racine, 2009; Gout, 2011). Studies that examine protective factors in the context of IPV perpetration measure strengths that are inverted risk factors as opposed to strengths the exist independently of risk. Being employed — versus being unemployed, a dynamic risk factor- - has been found to mitigate repeat IPV perpetration, for instance (Fanslow \& Gulliver, 2015). Given that DRAOR Protective items do not represent inverted risk factors, there is currently no research to indicate how they might perform when used to predict IPV recidivism. However, results for the effects of protective factors on violent recidivism can be used to make hypotheses about repeat IPV, given the violent nature of these types of crimes.

Ulrich and Coid (2011) found that social support, emotional support, involvement in religious activities, and closeness with others significantly reduced the likelihood of violent reconviction. Social skills, relationships, and self-care were significantly related to reduced violence in Abidin and colleagues' (2013) research, and Yoon et al. (2018) found that life goals was a significant predictor of desistance from violent recidivism. Of these items, social support, closeness with others, and relationships map onto the DRAOR Protective social support item. This item considers whether offenders have any meaningful relationships with non-criminal individuals (e.g., partners, family members, friends).

\section{Iowa Risk Assessment}

The Iowa Risk Assessment (IRA) was developed by the Iowa Department of Corrections 
(IDOC) to assist evaluators in determining offenders' level of community supervision (see Appendix D; IDOC, 2003). It is a 13-item actuarial instrument comprised solely of static risk items and is used in routine correctional assessments in Iowa, often utilized to predict multiple community outcomes. Serin, Chadwick, and Prell (2018) examined the predictive validity of the IRA when predicting time to first technical violation or new charge using univariate Cox regression survival analyses. The IRA significantly predicted time to both community outcomes.

\section{The Current Study}

Intimate partner violence is a serious public health issue that negatively impacts women's well-being and has considerable cost implications. Effective risk assessments and interventions help to reduce repeat IPV against women, serving to ameliorate this worldwide threat to women's health and safety. The DRAOR and IRA are used together in Iowa as routine case management and risk measures for reducing and predicting recidivism among all communitybased adult offenders. As mentioned previously, the DRAOR has been validated on samples of general, high-risk, and sex offenders; however, the instrument has yet to be validated on IPV offenders. Given the statewide implementation of the DRAOR in routine correctional assessments (i.e., its current use with IPV offenders in Iowa) and the prevalence of IPV globally, as well as in the U.S., it is important to determine whether the instrument can be usefully applied to IPV offenders.

Determining the utility of the DRAOR in predicting IPV recidivism will allow for its application to a wider range of community outcomes if it proves to be a scalable tool. Notably, the aim of the current study is not to advocate for the use of the DRAOR over validated IPVspecific risk tools, such as the ODARA, DVSI, or SARA, but rather to advocate for its use in the absence of such specialized instruments if it proves to accurately predict repeat IPV. The current 
study therefore seeks to validate the DRAOR for use with IPV offenders for various recidivism outcomes, with a focus on repeat IPV. The following discussion describes key research questions the present study aims to address, as well as corresponding hypotheses.

Research Question 1a: Do DRAOR Total, Stable, Acute, and Protective scores, as well as individual item scores differ between IPV and non-IPV offenders?

Hypothesis 1a: IPV offenders are expected to score higher than non-IPV offenders on the Acute subscale, the Stable items impulse control and attachment with others, and the following Acute items: substance abuse, anger/hostility, opportunity/access to victims, negative mood, employment, and interpersonal relationships. IPV offenders are also expected to score lower than non-IPV offenders on the social support item in the Protective domain. No predictions regarding the DRAOR Total, Stable and Protective domains, or the remaining items are made given the exploratory nature of these analyses. Research Question 1b: Do the base rates for recidivism vary across offender typology (IPV versus non-IPV) and DRAOR risk level (Low, Moderate, Moderate/High, High)? Hypothesis $1 b$ : IPV offenders will have higher violent recidivism base rates than non-IPV offenders due to the violent nature of their crimes.

No predictions regarding risk level or the other outcomes are made.

Research Question 1c: Do more IPV offenders fall into higher DRAOR risk levels than non-IPV offenders?

This research question is exploratory, thus no hypotheses are made.

These first three research questions may be helpful in advancing theory on IPV and may have important implications for practice. Namely, the ecological model for violence perpetration includes both individual and contextual factors (Dahlberg \& Krug, 2002), and DRAOR items can 
be shown to fit this model. Individual variables include attitudes towards authority, impulse control, problem solving, sense of entitlement, attachment with others, ${ }^{17}$ substance abuse, anger/hostility, negative mood, responsive to advice, prosocial identity, high expectations, costs/benefits, and employment. ${ }^{18}$ Relationship variables include peer associations, attachment with others, ${ }^{19}$ opportunity/access to victims, interpersonal relationships, social support, and social control. Lastly, employment ${ }^{20}$ and living situation ${ }^{21}$ represent community variables (note that the DRAOR does not include any societal variables; see Appendix B for a detailed description of the scale's items). Different item scores across offender typology may provide additional support for the multidimensional explanation of IPV. For example, IPV offenders scoring higher than non-IPV offenders on these individual, relationship, and community factors would lend support to the theory that IPV is the result of numerous individual and contextual factors, rather than a single factor type. In terms of practical implications, different domain and item scores across offender typology would indicate which factors are important intervention targets for IPV offenders and inform differentiated case planning and risk-management strategies. Differences in risk levels would indicate whether IPV offenders generally need more or less intensive supervision than non-IPV offenders.

The DRAOR has demonstrated sufficient psychometric properties with previous samples of general, high-risk, and sex offenders. The psychometric properties of particular interest in the

\footnotetext{
${ }^{17}$ Although it may appear that attachment with others is a relationship-only variable, it partly focuses on a lack of concern for others - an individual variable - that results in social disconnection or problematic interpersonal attachments, thus it also qualifies as an individual variable.

${ }^{18}$ Because this variable focuses on personal employment factors like skills, job satisfaction, and motivation to gain/maintain employment, it also qualifies as an individual variable as opposed to a community-only variable. ${ }^{19}$ In addition to being classified as an individual variable, attachment with others is also a relationship variable because it recognizes the nature of relationships with others, such as short-term or superficial relationships.

${ }^{20}$ In addition to being classified as an individual variable, employment is also considered a community variable because it is concerned with employment status/type.

${ }^{21}$ Because this variable primarily assesses if the offender is currently living in stable, long-term housing, it is considered a community variable.
} 
current study are reliability and convergent/divergent validity. Note that inter-rater reliability was not examined because the dataset used in this research does not support this type of reliability analysis.

Research Question 2: Does the DRAOR demonstrate adequate psychometric properties (i.e., reliability and convergent/divergent validity) with IPV offenders?

Hypothesis 2: The DRAOR will demonstrate adequate psychometric properties (i.e., reliability and convergent/divergent validity) with IPV offenders.

The DRAOR has demonstrated significant predictive accuracy for general recidivism and parole violations among diverse offender groups, including general, high-risk, and sex offenders. Several risk factors identified in the literature as significant predictors of IPV recidivism are items in the Acute subscale (i.e., substance abuse, anger/hostility, opportunity/access to victims, negative mood, employment, and interpersonal relationships). Thus, if the DRAOR predicts IPV recidivism, the Acute subscale is likely to account for much the instrument's predictive utility for this outcome. There is limited validation research on the IRA, however, a recent study found the scale to be a significant predictor of both time to general recidivism and technical violations (Serin et al., 2018). As DRAOR risk levels have not been examined empirically, this study seeks to provide initial validation information on their use with IPV and non-IPV offenders (together and separately) in hopes of determining their utility in case management.

Research Question 3a: Do the DRAOR Total and domains accurately predict general recidivism, IPV recidivism, violent recidivism, and technical violations among IPV offenders?

Hypothesis 3a: The DRAOR Total will predict general recidivism, IPV recidivism, violent recidivism, and technical violations among IPV offenders. The Acute subscale 
will predict IPV recidivism among IPV offenders. No predictions regarding the other subscales are made.

Research Question 3b: Do individual DRAOR items accurately predict IPV recidivism among IPV offenders?

Hypothesis 3b: The Stable items impulse control and attachment with others and Acute items substance abuse, anger/hostility, opportunity/access to victims, negative mood, employment, and interpersonal relationships will predict IPV recidivism among IPV offenders. The Protective item social support will predict IPV desistance among IPV offenders. No predictions regarding the other items are made.

Research Question 3c: Is the IRA an accurate predictor of general recidivism, IPV recidivism, violent recidivism, and technical violations among IPV offenders?

Hypothesis 3c: The IRA will predict general recidivism and technical violations among IPV offenders. No predictions regarding how it will perform when predicting IPV or violent recidivism are made.

Research Question 3d: Does the probability of general recidivism, IPV recidivism, violent recidivism, and technical violations increase as IPV and non-IPV offenders move up in DRAOR risk level?

Hypothesis $3 d$. The probability of recidivism and technical violations will increase as IPV and non-IPV offenders move up in DRAOR risk level.

The DRAOR has demonstrated the ability to predict general and violent recidivism, as well as parole violations over static risk. The Acute subscale has also been shown to predict general and violent recidivism over static risk. Additionally, five of the seven Acute items have consistently been identified in the literature as significant predictors of IPV. 
Research Question 4: Do the DRAOR Total, Stable, Acute, and Protective subscales improve the prediction of time to general recidivism, IPV recidivism, violent recidivism, and technical violations among IPV offenders beyond static risk (i.e., the IRA)? Hypothesis 4a: The DRAOR Total will predict time to general recidivism, IPV recidivism, violent recidivism, and technical violations among IPV offenders over and above static risk (i.e., the IRA).

Hypothesis $4 b$ : The Acute subscale will predict time to IPV and violent recidivism among IPV offenders over and above static risk (i.e., the IRA). No predictions regarding the other DRAOR domains or outcomes are made.

\section{Method}

\section{Participants}

This dataset is from the pilot implementation of the DRAOR in the state of Iowa and has been used in a previous validation study $(N=562$; for additional information, see Serin et al., 2018). The current sample includes a total of 112 male offenders with a previous, index, and/or recidivistic IPV offence who were serving community supervision orders between May 8, 2006 and November 16, 2015. Participants' demographic information is presented in Table 3. More than half the sample was Caucasian (56.25\%) and 95.54\% of offenders identified as nonHispanic. The majority of the sample was single (60.71\%) and most offenders' highest level of education was high school (73.21\%). Increasingly, there is interest in examining the gender of both offenders and victims, however, information on sexual orientation for the present sample was unavailable.

A large portion of offenders were being supervised on probation orders $(83.93 \%$; i.e., serving a community sentence as an alternative to incarceration), while the remaining sample 
was on some form of community supervision, such as work release (i.e., temporary release from prison to maintain employment), parole (i.e., a community sentence after some period of incarceration), or pretrial release (i.e., bail). The average age at the time of the initial DRAOR assessment was $34.58(S D=10.57)$. The majority of the sample received DRAOR Total scores that categorized them into the two lowest risk levels (37.50\% Low and 35.71\% Moderate). Conversely, most of the sample received IRA scores that placed them into the two highest supervision levels (54.46\% Intensive and $24.11 \%$ High Normal). One third of the sample (33.93\%) was convicted of a felony offence and a large portion $(29.46 \%)$ was convicted of an aggravated misdemeanor, the most serious class of misdemeanors.

Preliminary analyses and those concerning DRAOR risk level examined differences between 112 male IPV offenders and 398 male non-IPV offenders. As mentioned above, nonIPV offenders include participants with no previous, convicting, or recidivistic IPV offence. Demographic information for non-IPV offenders is presented in Table 3. Approximately threequarters of this sample was Caucasian $(75.13 \%)$ and $96.23 \%$ of offenders identified as nonHispanic. The majority of this sample was single (59.05\%) and most offenders' highest level of education was high school (59.30\%). A large portion of offenders were being supervised on probation orders $(80.15 \%)$, with the remainder on work release, parole, or bail. The average age at the time of the first DRAOR assessment was $31.70(S D=12.49)$. The majority of the non-IPV sample received DRAOR Total scores that categorized them into the two lowest risk levels (43.97\% Low and 39.45\% Moderate). Conversely, most of this sample received IRA scores that placed them into the highest supervision level (44.47\% Intensive). Roughly half of the non-IPV sample $(53.02 \%)$ was convicted of a felony offence and slightly less than a quarter of this sample (21.11\%) was convicted of an aggravated misdemeanor. 
Table 3

Demographic Information for IPV and Non-IPV Offenders

\begin{tabular}{|c|c|c|}
\hline & IPV & Non-IPV \\
\hline Variable & $n(\%)$ & $n(\%)$ \\
\hline \multicolumn{3}{|l|}{ Race } \\
\hline Black & $49(43.75)$ & $98(24.62)$ \\
\hline Caucasian & $63(56.25)$ & $299(75.13)$ \\
\hline Asian/Pacific Islander & $0(0.00)$ & $1(0.25)$ \\
\hline \multicolumn{3}{|l|}{ Ethnicity } \\
\hline Hispanic & $4(3.57)$ & $15(3.77)$ \\
\hline Non-Hispanic & $107(95.54)$ & $383(96.23)$ \\
\hline \multicolumn{3}{|l|}{ Marital status } \\
\hline Divorced & $9(8.04)$ & $39(9.80)$ \\
\hline Married & $21(18.75)$ & $62(15.58)$ \\
\hline Single & $68(60.71)$ & $235(59.05)$ \\
\hline Common-law & $2(1.78)$ & $5(1.26)$ \\
\hline Unknown & $12(10.71)$ & $37(9.30)$ \\
\hline \multicolumn{3}{|l|}{ Highest education level } \\
\hline Less than high school $^{\mathrm{a}}$ & $22(19.64)$ & $95(23.87)$ \\
\hline High school diploma/GED & $82(73.21)$ & $236(59.30)$ \\
\hline No diploma/certificate & $2(1.78)$ & $7(1.76)$ \\
\hline Some college ${ }^{b}$ & $2(1.78)$ & $11(2.77)$ \\
\hline College degree $^{c}$ & $0(0.00)$ & $15(3.77)$ \\
\hline Technical training completion ${ }^{\mathrm{d}}$ & $1(0.89)$ & $8(2.01)$ \\
\hline Special education diploma & $0(0.00)$ & $5(1.26)$ \\
\hline Unknown & $3(2.68)$ & $20(5.03)$ \\
\hline \multicolumn{3}{|l|}{ Supervision status } \\
\hline Parole $^{\mathrm{e}}$ & $7(6.25)$ & $23(5.78)$ \\
\hline Probation $^{\mathrm{f}}$ & $94(83.93)$ & $319(80.15)$ \\
\hline Work release & $10(8.93)$ & $34(8.54)$ \\
\hline Pretrial release with supervision & $1(0.89)$ & $22(5.53)$ \\
\hline \multicolumn{3}{|l|}{ DRAOR risk level } \\
\hline Low & $42(37.50)$ & $175(43.97)$ \\
\hline Moderate & $40(35.71)$ & $157(39.45)$ \\
\hline Moderate/High & $29(25.89)$ & $65(16.33)$ \\
\hline High & $1(0.89)$ & $1(0.25)$ \\
\hline \multicolumn{3}{|l|}{ IRA supervision level } \\
\hline Administrative & $5(4.46)$ & $28(7.04)$ \\
\hline Minimum & $2(1.78)$ & $57(14.32)$ \\
\hline
\end{tabular}


Low Normal

High Normal

Intensive

Offence severity

Simple misdemeanor

Serious misdemeanor

Aggravated misdemeanor

Felony $^{\mathrm{g}}$

Special sentence $(2005)^{\mathrm{h}}$

NCIC virtual code ${ }^{\mathrm{i}}$
$17(15.18)$

$27(24.11)$

$61(54.46)$

$1(0.89)$

$23(20.54)$

$33(29.54)$

$48(33.93)$

$6(5.36)$

$1(0.89)$
$70(17.59)$

$66(16.58)$

$177(44.47)$

$4(1.01)$

$84(21.11)$

$211(53.02)$

54 (13.57)

$10(2.51)$

Note. IPV = intimate partner violence; DRAOR = Dynamic Risk Assessment for Offender Re-Entry; IRA = Iowa Risk Assessment.

${ }^{\mathrm{a}}$ Includes grades 7, 9, 10, 11, and $12 .{ }^{\mathrm{b}}$ For IPV offenders: includes those who were in college at the time of assessment and those who had completed their sophomore year of college; for non-IPV: includes those in college at the time of assessment, and those who completed their freshman, junior, or sophomore level college. 'Includes associate's, bachelor's, and master's degrees. 'Includes also vocational program/technical certificate. ${ }^{\mathrm{e}}$ Includes Interstate Compact Parole for non-IPV offenders only. ${ }^{\mathrm{f}}$ Includes Special Sentence and Interstate Compact Probation offenders. ${ }^{\mathrm{g}}$ Includes B, C, D class Felonies, other Felonies (non-IPV offenders only), and Enhancement to Original Penalty. ${ }^{\text {ha' }}$ In addition to any other punishment provided by law, sex offenders convicted of a misdemeanor or class $\mathrm{D}$ felony must be sentenced to a ten-year Special Sentence. Those convicted of a class C felony or greater offense receive a Special Sentence of life. The offender begins serving the Special Sentence as if on parole" (Prison Legal News, 2013, para. 2). ${ }^{\mathrm{i}}$ Offence code from the National Crime Information Centre (information on the type of code was unavailable).

\section{Measures}

DRAOR. The DRAOR is a structured, interview-based case management scale that examines six Stable and seven Acute risk factors, as well as six Protective factors. These domains are empirically related to an elevated risk of reoffending (Stable and Acute) and crime 
desistance (Protective). The DRAOR's theoretical underpinnings are rooted in an integrated model of offender re-entry that presents a life-course perspective, incorporating crime acquisition and desistance factors (for a depiction and a more detailed description of this model, see Serin, Lloyd, \& Hanby, 2010). Namely, the age-crime curve forms the basis of the model, with risk and desistance factors on opposite ends of the curve, illustrating that the processes for entering and ending crime are distinct. The risk factors for crime acquisition included in the model are young age, the Big Four (antisocial attitudes, antisocial personality, antisocial history, antisocial associates; Andrews \& Bonta, 2010), and substance misuse. Initiation of the change process is marked by the commitment to change, which could be due to an internal switch resulting from a single event or the culmination of events, or an external influence, such as prosocial supports, successful treatment, or a positive mentor. Serin and colleagues (2010) argue, however, that this commitment alone is not adequate to sustain change. Instead, desistance occurs through (1) the commitment to change, (2) the interplay of internal and external change factors, and (3) desistance correlates.

Internal change factors represented in the model include agency (believing in one's capabilities of influencing him/herself and the environment), attributions (whether an individual uses internal or external factors to explain events), outcome expectancies (the expected consequences of one's behaviour), identity (self-perception), and change beliefs (beliefs about one's ability to change, as well as the advantages and disadvantages of desisting versus maintaining active criminality). External change factors include correctional interventions, proactive supervision (principles of $\mathrm{CCP}$, such as developing working relationship with clients, focusing on risk and need, having skills to address criminal thinking), aftercare (frequency, quality, support), positive relationships, and a supportive community. Finally, desistance 
correlates include older age, high quality marriage, stable employment, changes in the crime costs and reward contingencies (i.e., viewing prosocial options more favorably than criminal activity), sobriety from substance use, and association with prosocial peers.

The DRAOR serves as a structured guide for community probation and parole officers to assess offenders during supervision contacts. Supervision officers focus on the three domains (Stable, Acute, Protective) as they complete their interviews and are provided with risk management strategies to address problem areas (e.g., referring the offender to Alcoholics/Narcotics Anonymous for a substance abuse issue). These domains and risk management strategies provide officers with an outline to consistently follow during supervision contacts. In considering these domain areas during interviews, probation officers review key information that should inform changes in risk state.

In addition to being selected for their relationships to reoffending risk and crime desistance, items were included in the DRAOR to allow the scale to detect changes in risk over time. Available research supports the use of the DRAOR for this purpose. Namely, in Hanby's (2013) research, individual-level changes in DRAOR scores over the two-year assessment period reflected community behaviour, such that as offenders remained crime-free, average Stable and Acute risk scores significantly decreased and average Protective scores significantly increased. Individual-level changes were also found in Serin and colleagues' (2016) work, with 85\% of the sample demonstrating changes in DRAOR Acute scores (half decreased) over the two-month assessment period (note that the authors did not report change patterns for Stable or Protective scores). In a study on adult and adolescent offenders, DRAOR Total, Stable, and Acute scores demonstrated significant decreases from the initial to last assessments, ${ }^{22}$ while Protective scores demonstrated significant increases (Ferguson, 2015). Reductions in risk were also significantly

\footnotetext{
${ }^{22}$ Assessment period not specified.
} 
related to crime desistance for both adults and adolescents (Ferguson, 2015). Similar results were reported by Muirhead (2016), who found that adolescent offenders made significant improvements over the assessment period ${ }^{23}$ (i.e., Total, Stable, and Acute scores decreased, while Protective scores increased). Reductions in risk also significantly predicted crime desistance (Muirhead, 2016). These results suggest that the DRAOR is sensitive to changes in risk over short and long time periods, and that changes in risk can accurately be used to predict recidivism.

While there are no specific questions in the DRAOR user-manual for scoring items, officers are provided with a series of prompt questions to help guide their interviews. All items are rated on a 3-point scale (0-2). Stable and Acute factors are rated as "not a problem" (0), "slight/possible problem" (1), or "definite problem" (2). Recall that items in the Stable domain address criminal orientation and impulsivity concerns (subscale scores range from 0 to 12 ), and items in the Acute domain address destabilizers and lifestyle stressors (subscale scores range from 0 to 14). Protective factors are rated as "not an asset" (0), "slight/possible asset" (1), or “definite asset" (2), and as mentioned previously, they address social support and prosocial identity changes (subscale scores range from 0 to 12). An item can be omitted if the evidence is unreliable, but officers are encouraged to consider a score of 1 in this case, rather than omitting an item solely because they are unsure about what score to apply. Given that the DRAOR is intended for use across supervision contacts, officers should be able resolve uncertainty and insufficient information with additional interview contact. The DRAOR takes approximately 20 to 30 minutes to score, depending on the evaluators' experience with the scale, the complexity of the case, and whether it is an initial or follow-up assessment.

Stable and Protective scores can be updated any time new, relevant information is learned about the offender. Stable items reflect ongoing engagement in criminal activity, therefore a

\footnotetext{
${ }^{23}$ Assessment period not specified.
} 
lower score should reflect progressive skill-building away from prior criminal orientation. Conversely, Protective items reflect ongoing efforts to desist from crime, therefore a higher score should reflect progressive engagement in activities that support a crime-free life. It should take 3-6 months for a client to demonstrate a one-point change in scores (e.g., definite problem to slight problem), and 6-12 months to demonstrate two-point change in scores (e.g., definite problem to not a problem). Acute scores need to be reconsidered at each supervision contact as these items reflect the most recent situation (i.e., a score can be changed in any direction at any time). Changes are based on the offender's behavior in the last week or two and if the most recent session occurred more than two weeks prior, preference is given for behavior in the past two weeks.

IRA. The IRA is a 13-item actuarial instrument that serves as a pre-screen for the DRAOR, providing structure to the DRAOR community-based case plan (IDOC, 2003). IRA items include (1) age at classification, (2) age at first adult conviction/juvenile adjudication, (3) prior juvenile commitments, (4) prior probation/parole supervisions, (5) number of prior probation/parole revocations, (6) felony/misdemeanor convictions, (7) misdemeanor conviction history, (8) sex, (9) alcohol usage problems, (10) drug usage problems, (11) number of address changes in the last 12 months, (12) companions, and (13) employment. Item scores are summed to reach a Total risk score ranging from -5 to 25 . Risk scores directly inform the recommended level of supervision (Administrative, Minimal, Low Normal, High Normal, Intensive). The IRA has a classification override feature that allows evaluators to use their discretion in altering supervision levels indicated by risk scores if there are extraneous variables to consider. 


\section{Procedure}

Community supervision officers with the Iowa Department of Corrections (IDOC) who volunteered for the pilot study received a one-day, in-class training session on August 25, 2010 delivered by Dr. Ralph Serin, the developer of the DRAOR. The pilot training emphasized the general literature of dynamic risk, the composition of the DRAOR, as well as scoring procedures. An officer advisory group was created to ensure that other trainees received support when required, and Ralph Serin was available to the advisory group to address issues that arose.

Officers began scoring the DRAOR during their supervision sessions once they received the training. Completed assessments were recorded in the form of file notes and entered in the Iowa Corrections Offender Network (ICON), which houses all relevant information pertaining to offenders involved in the IDOC. While assessments most proximal to recidivism have the highest predictive accuracy, the current dataset contains initial assessments only, hence analyses are based on these DRAOR ratings. Initial assessments were completed between March, 2011 and July, 2011 for the sample of offenders. IDOC staff retrieved demographic information, DRAOR scores, and collected recidivism data once assessments were completed.

Officers complete IRA forms after initial face-to-face contact with offenders. They utilize client/officer interviews, pre-sentence investigation information, probation/parole intake information, institutional reports, and other materials when completing the risk scale (IDOC, 2003). The IRA assessment completed closest to the date of the initial DRAOR assessment was retained for use in this study. Static assessments were completed within an average of approximately 5 months of initial DRAOR assessments $(M=4.85, S D=13.17){ }^{24}$

Follow-up time was calculated as the time between the offender's initial DRAOR assessment and the first date of any subsequent new charge, IPV charge, violent charge, or

\footnotetext{
${ }^{24}$ Note that data were missing for 17 cases.
} 
technical violation, or November 16, 2015, the study end-date. The time between the initial DRAOR assessment and any new charge or technical violation was calculated by Nick Chadwick for the original evaluation of the overall pilot dataset (Serin et al., 2018). For the current study, Microsoft Excel Version 15.30 was used to calculate the follow-up time in the community (in months for descriptive purposes and in days for survival analyses) until IPV and violent recidivism by subtracting the date of offender's initial DRAOR assessment from the date of their first IPV charge, violent charge, or the study end-date if the offender did not incur a new IPV or violent charge. This results in a slight underestimation of months to IPV and violent recidivism because Excel's threshold for one month is 31 days; any number of days that does not exceed this threshold is considered zero months (e.g., Excel considers 1 year, 4 months, and 31 days to be 16 months as opposed to 17 months because the number of days does not exceed 31 ). This is not an issue when predicting recidivism because analyses involve either a dichotomous variable or time to recidivism in days.

Previous and index (i.e., current) IPV offences were coded by comparing offenders' supervision start date to their IPV sentence date. Convictions that occurred before the supervision start date were coded as either history or index offences (note that the type of supervision was used to categorize offences. For example, convictions that occurred before the supervision start date were coded as previous offences for probationers, but were coded as either previous or current offences for parolees, depending on the length of time between sentencing and supervision dates), convictions that occurred on the same day as the supervision start date were coded as index offences, and convictions that occurred after the supervision start date were coded as recidivism. IPV history offences were the most common, followed by index offences, and then recidivism offences (see Table 4). 
Table 4

Number of History, Index, and Recidivism IPV Offenders and Incidents

\begin{tabular}{lcc}
\hline Offence type & No. offenders & No. incidents \\
\hline History & 68 & 111 \\
Index $_{\text {Recidivism }}{ }^{\mathrm{a}}$ & 47 & 50 \\
R & 26 & 29 \\
\hline
\end{tabular}

${ }^{a}$ Seven offenders (accounting for nine incidents) recidivated after their supervision start date, but before their DRAOR assessment date. These cases were treated as history offences given that the DRAOR is a case management tool used to identify and target dynamic risk factors, several of which change within hours or days. In other words, due to the dynamic nature of DRAOR items, the current study is interested in how well the DRAOR predicts prospective recidivism (i.e., recidivism that occurs after the first DRAOR assessment), not retrospective recidivism.

\section{Outcome Data}

Community outcomes included general recidivism, IPV recidivism, violent recidivism, and technical violations. Technical violations are the mildest form of recidivism, whereby an offender breaches a condition of their community supervision (e.g., failure to report to their supervision officer or abstain from substances). Official records were used to indicate whether an offender incurred any new charge (excluding technical violations), an IPV charge, a violent charge, or violated any of their supervision conditions throughout the study period. General recidivism, technical violations, and offence severity were coded by Nick Chadwick for the original evaluation of the overall pilot dataset (Serin et al., 2018). For new charges (i.e., general recidivism), both the presence and type (i.e., public order, property, drug, violent, other) of new charge was recorded. In the case of multiple charges occurring on the same date, the most severe charge was recorded. If multiple technical violations were recorded throughout the follow-up 
period, the earliest event was retained. All technical violations were also compiled to indicate the total number of instances an offender violated their conditions.

Severity was assessed based on whether the first charge was a felony or misdemeanour. ${ }^{25}$ IPV recidivism was coded by comparing offenders' first DRAOR assessment to their IPV sentence date (i.e., IPV convictions that occurred after the assessment date were coded as recidivism). Violent recidivism was coded from six offence variables, each representing separate charges during the follow-up, to ensure that violent offences were not missed due to a nonviolent first charge. IPV recidivism includes domestic assault. ${ }^{26}$ Violent recidivism includes assault (domestic and nondomestic), theft, sex offences, child endangerment, harassment, interference with official acts, weapons-related offences, and robbery. IPV and violent recidivism were recorded as dichotomous variables (yes/no) for the purpose of this study. ${ }^{27}$

The average follow-up time in the community until an event, as well as base rates are presented in Table 5. The follow-up time ranged from 0 days to approximately 4.5 years. An average of $7.64(S D=10.33), 8.25(S D=11.35)$, and $7.46(S D=10.04)$ violations were recorded throughout each offender's supervision period for the overall sample, IPV offenders, and nonIPV offenders, respectively.

\footnotetext{
${ }^{25}$ This does not account for later, more severe charges incurred by offenders, however, the present study only uses offence severity for descriptive purposes, therefore this is not a concern.

${ }^{26}$ Domestic assault charges most commonly involve intimate partners, however, information on whether participants offended against children or an intimate partner was not available, therefore IPV recidivism could include both victim types.

${ }^{27}$ Information on whether participants offended against the same or a new victim was not available, therefore IPV recidivism could include both old and new victims.
} 
Table 5

Follow-up Time in Months (Ms and SDs) and Outcome Base Rates for Overall Sample, IPV, and Non-IPV Offenders

\begin{tabular}{|c|c|c|c|c|c|c|}
\hline \multirow[b]{2}{*}{ Outcome } & \multicolumn{2}{|c|}{ Overall sample } & \multicolumn{2}{|c|}{ IPV offenders } & \multicolumn{2}{|c|}{ Non-IPV offenders } \\
\hline & $M(S D)$ & $\%(n)$ & $M(S D)$ & $\%(n)$ & $M(S D)$ & $\%(n)$ \\
\hline General recidivism & $40.67(19.16)$ & $38.04(194)$ & $34.72(21.20)$ & $52.68(59)$ & $42.34(18.23)$ & $33.92(135)$ \\
\hline IPV recidivism & $52.08(8.52)$ & $5.10(26)$ & $45.80(16.67)$ & $23.21(26)$ & & \\
\hline Violent recidivism & $49.58(12.46)$ & $13.14(67)$ & $42.48(18.48)$ & $35.71(40)$ & $51.57(9.26)$ & $6.78(27)$ \\
\hline Technical violations & $23.23(23.81)$ & $64.90(331)$ & $21.88(23.67)$ & $70.54(79)$ & $23.61(23.86)$ & $63.32(252)$ \\
\hline
\end{tabular}

Note. IPV = intimate partner violence. 


\section{Data Cleaning}

Initial data cleaning was completed by Nick Chadwick for the original evaluation of the overall pilot dataset $(N=562$; Serin et al., 2018). The author examined all variables for data entry errors and the presence of missing data. Five cases contained out-of-range DRAOR item values. The original responses were unavailable to facilitate the cross-comparison of scores, thus these cases were removed from the overall pilot dataset. However, note that four of these were non-IPV cases, so the primary analyses in the current study were only affected by the removal of one case. There were 104 offenders (18.51\%) in the overall pilot dataset who had an identified supervision level according to the IRA, but did not have a specified total risk score. The mean value for these offenders' corresponding risk bin was inserted into their IRA Total score to allow

for inclusion in analyses. Serin and colleagues (2018) found no significant differences across the demographic variables when comparing the 104 offenders who did not have original IRA scores with those who did. Approximately the same percentage of IPV as non-IPV offenders were missing an IRA risk score $(15.1 \% ; n=17)$. Aside from missing ethnicity information for one case, there were no missing data across demographic variables or DRAOR items. Differences in ethnicity are not a primary interest of this study, therefore the missing information for one case is not a concern.

Offenders in the original dataset $(N=562)$ were grouped into IPV (those with a criminal history, index, or recidivism offence related to IPV; $n=117)$ and non-IPV $(n=445)$ samples. There were not enough female IPV offenders $(n=5)$ to facilitate comparisons across gender, therefore these participants were removed from the dataset, resulting in a total IPV sample of 112 male offenders. Female non-IPV offenders $(n=47)$ were also removed from the dataset to 
facilitate comparisons with male IPV offenders, resulting in a total non-IPV sample of 398 male offenders.

\section{Overview of Analyses}

Research Questions 1a, 1b, and 1c examine differences between 112 male IPV offenders and 398 male non-IPV offenders. Additionally, Research Question 3d examines whether the probability of recidivism and technical violations increases as IPV and non-IPV offenders move up in DRAOR risk level. The remaining research questions concern IPV offenders only. Microsoft Excel Version 15.30 was used to compute Cohen's $d$ s for Question 1a, to analyze Question 1c, and to determine expected probabilities of recidivism and technical violations for Question 3d. The remaining research questions were analyzed using SPSS Version 24.

Differences between IPV and non-IPV offenders. Research Questions 1a, 1b, and 1c examined differences in DRAOR scores, recidivism base rates, and DRAOR risk levels across offender typology.

Research Question 1a: Do DRAOR Total, Stable, Acute, and Protective scores, as well as individual Stable and Acute item scores differ between IPV and non-IPV offenders?

In addition to descriptive statistics, analyses include area under the curve (AUC) statistics from receiver operating characteristic (ROC) curve analyses and 95\% confidence intervals (CIs). The AUC is an effect size statistic that is appropriate when one variable is dichotomous and the other is dichotomous, ordinal, or interval (Swets, Dawes, \& Monahan 2000). AUCs are one of the most commonly recommended effect size statistics for examining relationships to recidivism (Helmus \& Babchishin, 2017), but are rarely used for dichotomous variables other than recidivism (Babchishin \& Helmus, 2016). Recent research, for example, has utilized AUCs in examining differences between Aboriginal and non-Aboriginal offenders (Babchishin, Blais, \& 
Helmus, 2012; Helmus, Babchishin, \& Blais, 2012; Perley-Robertson, Helmus, \& Forth, 2018). An AUC in this context is interpreted as the "probability that a randomly selected Aboriginal offender will have a higher risk score than a randomly selected non-Aboriginal offender" (Babchishin \& Helmus, 2016, p. 1023).

Notably, AUCs are robust to attenuations in magnitude due to high or low base rates (i.e., deviations from a $50 \%$ base rate) on dichotomous outcome variables, such as the endorsement rate on the proportion of a subgroup in a sample (e.g., the proportion of Aboriginal versus nonAboriginal offenders; Babchishin \& Helmus, 2016). This is important for the current research question, examined differences in DRAOR Total, domain, and item scores between 112 (21.96\%) IPV offenders and 398 (78.04\%) non-IPV offenders.

AUCs were used to predict offender typology for this research question (i.e., to determine if DRAOR Total, domain, and item scores were associated with being an IPV offender). AUCs can be interpreted as probability that a randomly selected IPV offender will have a higher risk or protective score than a randomly selected non-IPV offender (note that risk scores are at the scale, subscale- and item-level, but protective scores are only at the subscale- and item-level). AUC values can vary between 0 and 1, with .50 indicating no difference in the outcome variable (i.e., offender typology). AUCs above .50 indicate that IPV offenders scored higher on the DRAOR Total, domains, and items, while AUCs below .50 indicate that IPV offenders scored lower. As a rough heuristic, AUC values of .56, .64, and .71 reflect small, moderate, and large effect sizes, respectively, as these values roughly correspond to Cohen's $d$ s of $.20, .50$, and .80 (Rice $\&$ Harris 2005). Conversely, AUCs of .44, .36, and .29 reflect small, moderate, and large effect sizes in the opposite direction. An AUC value is statistically significant if the $95 \%$ CI does not include .50 . 
Although it may appear that a Student's independent $t$-test would be more appropriate for addressing this research question, the AUC statistic was used because it simultaneously tests whether there is a difference and provides an effect size. Additionally, if the assumptions of the Student's $t$-test are violated (e.g., homogeneity of variance), non-parametric tests are used instead. As the AUC is a non-parametric test, violating assumptions is not of concern.

Effect size was measured using Cohen's $d$ s as follows:

$$
\begin{gathered}
d=\frac{M_{1}-M_{2}}{S D_{\text {pooled }}} \\
S D_{\text {pooled }}=\sqrt{\frac{S D_{1}^{2}+S D_{2}^{2}}{2}}
\end{gathered}
$$

Research Question 1b: Do the base rates for recidivism vary across offender typology (IPV versus non-IPV) and risk level (Low, Moderate, Moderate/High, High)?

Research Question 1c: Do more IPV offenders fall into higher DRAOR risk levels than non-IPV offenders?

Descriptive statistics were used to determine base rates for general recidivism, IPV recidivism, violent recidivism, and technical violations across offender typology and risk level. To test whether IPV offenders were rated as lower or higher risk on the DRAOR, ordinal test statistic, $M^{2}$, the linear trend alternative to the Pearson's chi-squared $\left(\chi^{2}\right)$ test of independence (Agresti, 2007) was used. Although it may appear that a Pearson's chi-squared test of independence is more appropriate for these data, the $\chi^{2}$ value in this test does not change as a function of the order in which the rows and columns are listed (i.e., this test treats both variables as nominal; Agresti, 2007). Tests of categorical variables that involve at least one ordinal variable are more appropriate — and can benefit from increased statistical power-when the 
ordinal variable is treated as quantitative, rather that qualitative (Agresti, 2007). The ordinal test statistic, $M^{2}$, which can be interpreted as $\chi^{2}$ on 1 degree of freedom $(d f)$, is appropriate for use when there is one dichotomous ${ }^{28}$ nominal variable and one ordinal variable (Agresti, 2007). It treats both categorical variables as ordinal by assigning ranked scores to categories, and measuring the degree of the linear trend between the two variables using Pearson's correlation $r$. Larger absolute $r$ values reflect a higher degree of dependence between the two variables (Agresti, 2007). The only assumption of $M^{2}$ is that observations are independent (Agresti, 2007). $M^{2}$ was calculated in Excel as follows:

1. $r:=\mathrm{CORREL}(\operatorname{array} 1, \operatorname{array} 2)$

Where array $1=$ offender typology and array $2=$ DRAOR risk level for the overall sample of 510 IPV and non-IPV offenders

2. $M^{2}:=(N-1) *\left(r^{\wedge} 2\right)$

Where $N=510$

3. $p$-value: $=\operatorname{CHIDIST}\left(M^{2}, d f\right)$

Where $d f=1$

Psychometrics. To validate the DRAOR for use with IPV offenders, Research Question 2 examined the scale's psychometric properties.

Research Question 2: Does the DRAOR demonstrate adequate psychometric properties (i.e., reliability and convergent/divergent validity) with IPV offenders?

Reliability analyses include Cronbach's alphas and item-total correlations. Cronbach's alpha is the most common method for assessing reliability (Field. 2009). It is essentially equivalent to splitting data for each individual subscale in half, every possible way, computing correlation coefficients for each pair of split-halves, and then taking the average of those

\footnotetext{
${ }^{28}$ This test is not appropriate when the nominal variable has more than two categories (Agresti, 2007).
} 
coefficients to produce an alpha value. If the scale is internally consistent, then offenders' scores on each of the split-half pairs should be highly correlated, indicating the scale/subscale consistently reflects the construct(s) that it measures. There is some debate in the field regarding the level of acceptability for Cronbach's alphas, however, it is commonly recommended that .70 (Reynolds \& Livingstone, 2012) or .80 (Clark \& Watson, 1995) serve as minimum criteria values for determining internal scale consistency, with the former being a more liberal criterion. Cronbach's alpha values 'if item is deleted' were examined to determine if there are any problematic items. If the removal of an item results in a substantially greater alpha than the overall subscale alpha, it is typically recommended that the item be deleted from the subscale to improve reliability (Field, 2009). Item-total correlations measure the degree of association between individual items and their respective subscale total score, with .30 serving as the minimum criterion for acceptable internal consistency (Field, 2009).

Inter-correlations between subscales, correlations between subscales and the IRA, as well as correlations between the DRAOR Total and IRA were examined to assess convergent and divergent validity. Significant positive correlations indicate that two scales and/or subscales measure similar constructs (i.e., convergent validity; the Stable and Acute subscales should be positively associated because they both measure dynamic risk, for example), while significant negative correlations indicate that two scales and/or subscales measure different constructs (i.e., divergent validity; the Protective subscale should be negatively associated will all other risk scales/subscales because it measures factors that protect against risk). Correlations of .10, .30 , and .50 represent small, moderate, and large effect sizes, respectively (Cohen, 1992).

Predictive validity. Research Questions $3 \mathrm{a}$ and $3 \mathrm{~b}$ examined the predictive validity of DRAOR scores among IPV offenders, Research Question 3c examined the predictive validity of 
the IRA among IPV offenders, and Research Question 3d examined whether increases in DRAOR risk level predicted community outcomes among IPV and non-IPV offenders.

\section{Research Questions 3:}

a) Do the DRAOR Total and domains accurately predict general recidivism, IPV recidivism, violent recidivism, and technical violations among IPV offenders?

b) Do individual DRAOR items accurately predict IPV recidivism among IPV offenders?

c) Is the IRA an accurate predictor of general recidivism, IPV recidivism, violent recidivism, and technical violations among IPV offenders?

d) Does the probability of general recidivism, IPV recidivism, violent recidivism, and technical violations increase as IPV and non-IPV offenders move up in DRAOR risk level?

Analyses for Research Questions 3a, 3b, and 3c include AUCs and 95\% CIs. As previously mentioned, AUCs are one of the most commonly recommended effect size statistics for examining relationships to recidivism (Helmus \& Babchishin, 2017). They can be interpreted as the "probability that a randomly selected recidivist has a higher score on the risk scale than a randomly selected non-recidivist” Babchishin \& Helmus, 2016, p. 1023). Importantly, AUCs have been found to be the most robust to attenuations in magnitude due to high or low base rates compared to correlations, other common variations of this statistic (e.g., Kendall's tau correlation), and common alternatives to correlations (e.g., Cohen's $d$; Babchishin \& Helmus, 2016).

AUCs were used to predict four community outcomes: general recidivism, IPV recidivism, violent recidivism, and technical violations. Recall that AUC values can vary 
between 0 and 1, with .50 indicating no difference in the outcome variable. AUCs above .50 indicate that higher DRAOR Total, domain, and item scores were positively associated with the outcome (i.e., higher rates of recidivism) and AUCs below .50 indicate that higher DRAOR Total, domain, and item scores were negatively associated with the outcome (i.e., lower rates of recidivism). AUCs of $.56, .64$, and .71 correspond to small, moderate, and large effect sizes, whereas AUCs of $.44, .36$, and .29 reflect small, moderate, and large effect sizes in the opposite direction, as mentioned above. An AUC value is statistically significant if the $95 \%$ CI does not include .50 .

A series of binary logistic regression analyses were utilized to answer Research Question 3d. Binary logistic regression is used to predict the probability of $Y$ occurring given known values of $X$ (Field, 2009). In the context of the current study, this involves predicting the probability of recidivism and technical violations for each DRAOR Total score. Logistic regression produces an odds ratio (OR), which indicates the change in the odds of recidivism and violations for a unit change in DRAOR Total score (Field, 2009). Notably, odds are distinct from probability in that the odds of recidivism occurring are equal to the probability of recidivating divided by the probability of not recidivating. Conversely, the probability of recidivism is equal to the number of recidivists divided by the total sample. For example, suppose 25 out of 100 offenders recidivated; the odds of recidivism are $(25 / 100) /(75 / 100)=.25 / .75=0.33$, whereas the probability of recidivism is $25 / 100=.25$, which can also be expressed as a percentage $(25 \%)$. Odds can range from 0 to infinity, whereas probabilities can range from 0 to 1.

An odds ratio greater than 1 indicates that the odds of recidivism and violations increase as the DRAOR Total score increases, whereas an odds ratio less than 1 indicates that the odds of recidivism and violations decrease as the DRAOR Total score increases. An odds ratio of 3.41 
indicates that the odds of recidivism are 3.41 times higher for each unit increase in DRAOR Total score, for example (Tabachnick \& Fidell, 2013). Conversely, an odds ratio of .41 indicates that the odds of recidivism are .41 times lower for each unit increase in DRAOR Total score (Tabachnick \& Fidell, 2013). ORs of 1.68, 3.47, and 6.71 reflect small, moderate, and large effect sizes, respectively, as these values roughly correspond to Cohen's $d$ s of .20, .50, and .80 (Chen, Cohen, Chen, 2010).

Expected recidivism rates were calculated using the following formula, where $B_{0}$ is the intercept, $B_{1}$ is the slope, and $X$ is a given DRAOR Total score:

$$
p=\frac{e^{\left(B_{0}+B_{1} X\right)}}{1+e^{\left(B_{0}+B_{1} X\right)}}
$$

This was done for IPV and non-IPV offenders for all applicable outcomes (i.e., excluding IPV recidivism for non-IPV offenders). Additionally, given that this is the first empirical study to examine expected recidivism rates, the above formula was also used to obtain recidivism estimates for the overall sample.

Incremental predictive validity. Research Question 4 aimed to examine the ability of the DRAOR to predict recidivism beyond static risk.

Research Question 4: Do the DRAOR Total, Stable, Acute, and Protective subscales improve the prediction of time to general recidivism, IPV recidivism, violent recidivism, and technical violations among IPV offenders beyond static risk (i.e., the IRA)? Univariate Cox regression survival analyses were used to examine the association between dynamic (DRAOR Total, Stable, Acute, and Protective subscales) and static variables (the IRA) and recidivism/technical violations while controlling for the time to community outcomes. Variables independently related to the time to an event will then be entered into a series of hierarchal Cox regression survival analyses to examine the incremental validity of the 
dynamic variables beyond static risk. The dependent variables are time-at-risk; namely, the number of days between the initial DRAOR assessment and either the first date of any subsequent new charge, IPV charge, violent charge, technical violation, or November 16, 2015 (the study end-date) if no new charges were incurred.

Regression coefficients (B) for these analyses are used to determine hazard ratios (HR), which provide an indication of how a particular covariate influences the risk of failure (i.e., recidivism/technical violations; Tabachnick \& Fidell, 2013). A positive regression coefficientand a hazard ratio greater than 1 -indicates that recidivism and technical violations occurred more quickly after release (i.e., increases in DRAOR Total, Stable, Acute, and Protective scores led to increases in likelihood of recidivating and violating supervision conditions; Tabachnick \& Fidell, 2013). Conversely, a negative regression coefficient — and a hazard ratio less than $1-$ indicates that recidivism and technical violations occurred later after release (i.e., increases in DRAOR Total, Stable, Acute, and Protective scores led to decreases in the likelihood of recidivating and violating supervision conditions; Tabachnick \& Fidell, 2013). A hazard ratio of 1.79 indicates the likelihood of recidivating and violating conditions increases by $79 \%$ ([[1$1.79 \mid] 100=79$ ) with each point increase in risk and protective scores, for example (Tabachnick \& Fidell, 2013). In contrast, a hazard ratio of .79 indicates the likelihood of recidivating and violating conditions decreases by $21 \%([|1-.79|] 100=21)$ with each point increase in risk and protective scores (Tabachnick \& Fidell, 2013).

\section{Results}

\section{Data Screening}

Logistic regression. This analysis assumes a linear relationship between continuous predictors and the logit transformation of the dependent variables (Tabachnick \& Fidell, 2013). 
To test this assumption, a constant of 13 was added to DRAOR Total scores so that no values were less than 1. The DRAOR Total and the interaction between this variable and its natural logarithm were then added to logistic regression models for each community outcome. None of the interaction terms were significant, indicating the linearity of the logit assumption is satisfied for all outcomes. Univariate outliers were examined through $z$-scores using an absolute critical value of 3.3 as the criterion (Tabachnick \& Fidell, 2013). No cases had $z$-scores exceeding 3.3. Further, outliers can be identified using DFFITS and DFBETAS, such that absolute values greater than 1 suggest a given case will influence the results (Kutner, Nachtsheim, Neeter, \& Li, 2005). All absolute DFFITS and DFBETAS values were below 1.

Cox regression survival analysis. Data were examined for multicollinearity, univariate and multivariate outliers, and proportionality of hazards. Multicollinearity was assessed using variance inflation factor (VIF) values, with 4 serving as the VIF criterion (Pan \& Jackson, 2008). Multicollinearity is not an issue in the present dataset, as all VIF values were less than 4. Univariate outliers were examined through $z$-scores using an absolute critical value of 3.3 as the criterion (Tabachnick \& Fidell, 2013). There were five univariate outliers for the IRA score, however, absolute DFFITS and DFBETAS values were below 1, indicating these outlying cases are not influential. Multivariate outliers were examined through Mahalanobis' distance using a critical $\chi^{2}$ value of $20.51(d f=5, \alpha=.001$; Tabachnick \& Fidell, 2013). No cases exceeded 20.51, thus there were no multivariate outliers.

The proportionality of hazards assumption states that the shape of the survival function over time is the same for all cases and groups (Tabachnick \& Fidell, 2013). Non-proportional hazards indicate there is an interaction between groups and time in survival rates, or an interaction between covariates and time (Tabachnick \& Fidell, 2013). The proportionality of 
hazards assumption was examined by creating a time variable and testing it for interactions with each covariate across all outcomes using Cox regression survival analyses with time-dependent covariates. The assumption was met for all variables, as interactions between time and each covariate across outcomes were nonsignificant.

\section{Differences Between IPV and Non-IPV Offenders}

Research Question 1a: Do DRAOR Total, Stable, Acute, and Protective scores, as well as individual item scores differ between IPV and non-IPV offenders?

Table 6 provides means and standard deviations for DRAOR scores across offender typology. A comparison of these descriptives shows that IPV offenders scored higher on the Stable and Acute domains, as well as their respective items. IPV offenders also scored lower on the Protective domain and six of these items (mean scores on the responsive to advice item were the same for both IPV and non-IPV offenders). Lastly, DRAOR Total scores were higher for IPV offenders compared to non-IPV offenders.

As shown in Table 7, IPV offenders scored significantly higher than non-IPV offenders on seven of the 23 possible comparisons. This includes the Stable item attachment with others $\left(\mathrm{AUC}=.57\right.$ and $d^{29}=0.26$, small effects), the Acute domain $(\mathrm{AUC}=.65$ and $d=0.52$, moderate effects), Acute items substance abuse (AUC $=.57$ and $d=0.27$, small effects), anger/hostility $(\mathrm{AUC}=.72$ and $d=0.92$, large effects), opportunity/access to victims (AUC $=.59$ and $d=0.39$, small effects), and interpersonal relationships (AUC $=.60$ and $d=0.39$, small effects), and the DRAOR Total $(\mathrm{AUC}=.58$ and $d=0.32$, small effects $)$

Comparisons of AUCs versus Cohen's $d$ s revealed that the magnitude of effect size was largely unaffected by the type of effect size measure used. Namely, AUCs were less than small for the Stable domain score and all Stable items, except for attachment with others (AUC $=.57$, a

\footnotetext{
${ }^{29}$ Cohen's $d$ s of $.20, .50$, and .80 represent small, moderate, and large effect sizes, respectively (Cohen, 1992).
} 
small effect). Cohen's $d$ s were less than small for the Stable domain score and all Stable items, except impulse control $(d=0.20$, a small effect $)$ and attachment with others $(d=0.26$, a small effect). AUCs and Cohen's $d$ s ranged from less than small to large for the Acute domain score and Acute items (effect size magnitude was comparable for all comparisons). Lastly, AUCs were less than small for the Protective domain score and all Protective items. Cohen's $d$ s were less than small for the Protective domain score and all Protective items, except costs/benefits $(d=$ 0.21, a small effect). To conclude, AUCs and Cohen's $d$ s were of equal magnitude except for the Stable impulse control item and the Protective costs/benefits items, for which AUCs were less than small and Cohen's $d$ s were small. 
Table 6

DRAOR Descriptives (Ms and SDs) for IPV and Non-IPV Offenders

\begin{tabular}{lcc}
\hline & IPV $^{\mathrm{a}}$ & Non-IPV $^{\mathrm{b}}$ \\
\cline { 2 - 3 } DRAOR variable & $M(S D)$ & $M(S D)$ \\
\hline Stable & $5.19(2.73)$ & $4.67(2.65)$ \\
Peer Associations & $1.03(0.58)$ & $0.96(0.54)$ \\
Attitudes Towards & $0.69(0.63)$ & $0.65(0.61)$ \\
Impulse Control & $1.04(0.68)$ & $0.91(0.64)$ \\
Problem Solving & $0.96(0.57)$ & $0.88(0.60)$ \\
Sense of Entitlement & $0.68(0.67)$ & $0.63(0.61)$ \\
Attachment with Others & $0.79(0.59)$ & $0.64(0.55)$ \\
Acute & $6.57(3.00)$ & $5.07(2.73)$ \\
Substance Abuse & $0.96(0.83)$ & $0.75(0.73)$ \\
Anger/Hostility & $1.09(0.74)$ & $0.48(0.58)$ \\
Opportunity/Access to & $0.90(0.76)$ & $0.63(0.60)$ \\
Negative Mood & $0.71(0.69)$ & $0.68(0.64)$ \\
Employment & $0.93(0.89)$ & $0.86(0.83)$ \\
Interpersonal Relationships & $1.11(0.68)$ & $0.86(0.60)$ \\
Living Situation & $0.88(0.66)$ & $0.81(0.64)$ \\
Protective & $6.43(2.72)$ & $6.76(2.84)$ \\
Responsive to Advice & $1.17(0.61)$ & $1.17(0.55)$ \\
Prosocial Identity & $1.04(0.50)$ & $1.10(0.55)$ \\
High Expectations & $1.12(0.64)$ & $1.16(0.65)$ \\
Costs/Benefits & $1.05(0.55)$ & $1.17(0.60)$ \\
Social Support & $1.14(0.64)$ & $1.17(0.62)$ \\
Social Control & $0.91(0.61)$ & $1.00(0.60)$ \\
Total & $5.33(7.40)$ & $2.98(7.37)$ \\
\hline
\end{tabular}

Note. DRAOR = Dynamic Risk Assessment for Offender Re-Entry; IPV = intimate partner violence.

${ }^{\mathrm{a}} N=112 \cdot{ }^{\mathrm{b}} N=398$. 
Table 7

Differences in DRAOR Scores (AUCs and Cohen's ds) Between IPV and Non-IPV Offenders

\begin{tabular}{|c|c|c|}
\hline DRAOR variable & $\mathrm{AUC}[95 \% \mathrm{CI}]$ & $d$ \\
\hline Stable & $.55[.49, .61]$ & 0.19 \\
\hline Peer Associations & $.53[.47, .59]$ & 0.12 \\
\hline Attitudes Towards Authority & $.51[.45, .57]$ & 0.06 \\
\hline Impulse Control & $.55[.49, .61]$ & 0.20 \\
\hline Problem Solving & $.54[.48, .60]$ & 0.14 \\
\hline Sense of Entitlement & $.52[.45, .58]$ & 0.08 \\
\hline Attachment with Others & $.57[.51, .63]$ & 0.26 \\
\hline Acute & $.65[.59, .71]$ & 0.52 \\
\hline Substance Abuse & $.57[.51, .63]$ & 0.27 \\
\hline Anger/Hostility & $.72[.66, .77]$ & 0.92 \\
\hline Opportunity/Access to Victims & $.59[.53, .66]$ & 0.39 \\
\hline Negative Mood & $.51[.45, .57]$ & 0.05 \\
\hline Employment & $.52[.46, .58]$ & 0.08 \\
\hline Interpersonal Relationships & $.60[.54, .66]$ & 0.39 \\
\hline Living Situation & $.53[.47, .59]$ & 0.11 \\
\hline Protective & $.47[.41, .53]$ & 0.12 \\
\hline Responsive to Advice & $.50[.44, .56]$ & 0.00 \\
\hline Prosocial Identity & $.47[.41, .53]$ & 0.11 \\
\hline High Expectations & $.48[.42, .54]$ & 0.06 \\
\hline Costs/Benefits & $.45[.39, .51]$ & 0.21 \\
\hline Social Support & $.49[.43, .55]$ & 0.05 \\
\hline Social Control & $.46[.40, .53]$ & 0.15 \\
\hline Total & $.58[.52, .64]$ & 0.32 \\
\hline
\end{tabular}

Note. Significant AUCs are bolded. AUCs below .50 indicate that higher scores (i.e. more

Protective factors) were associated with lower rates of recidivism. DRAOR $=$ Dynamic Risk

Assessment for Offender Re-Entry; AUC = area under the curve statistic; $d=$ Cohen's $\mathrm{d}$.

Research Question 1b: Do the base rates for recidivism vary across offender typology (IPV versus non-IPV) and DRAOR risk level (Low, Moderate, Moderate/High, High)?

Outcome base rates for IPV and non-IPV offenders across the four DRAOR risk levels are presented in Table 8. Overall, a greater percentage of IPV offenders committed a new general 
offence, violent offence, and violated a supervision order $(52.68 \%, 35.71 \%$, and $70.54 \%$, respectively) during the follow-up period compared to non-IPV offenders $(33.92 \%, 6.79 \%$, and $63.32 \%$, respectively). Within each risk level, more IPV offenders committed a new general offence than non-IPV offenders, with the most marked difference in base rates found for Moderate/High risk offenders (15.18\% of IPV offenders versus 5.28\% of non-IPV offenders recidivated). The same was true for violent recidivism, with Moderate risk offenders exhibiting the largest difference (16.07\% of IPV offenders versus $3.77 \%$ of non-IPV offenders recidivated). More Low, Moderate/High, and High risk IPV offenders incurred a technical violation compared to non-IPV offenders, but fewer Moderate risk IPV offenders incurred a technical violation compared to non-IPV offenders (23.21\% versus 29.90\%, respectively). Moderate/High risk offenders had the largest base rate difference for technical violations (23.21\% for IPV offenders versus $14.07 \%$ for non-IPV offenders). 
Table 8

Outcome Base Rates Across Risk Level for IPV and Non-IPV Offenders

\begin{tabular}{|c|c|c|c|c|c|c|c|}
\hline \multirow[b]{3}{*}{ DRAOR risk level } & \multicolumn{4}{|c|}{$\mathrm{IPV}^{\mathrm{a}}$} & \multicolumn{3}{|c|}{ Non-IPV ${ }^{b}$} \\
\hline & $\begin{array}{c}\text { General } \\
\text { recidivism }\end{array}$ & IPV recidivism & $\begin{array}{c}\text { Violent } \\
\text { recidivism }\end{array}$ & $\begin{array}{l}\text { Technical } \\
\text { violations }\end{array}$ & $\begin{array}{c}\text { General } \\
\text { recidivism }\end{array}$ & $\begin{array}{c}\text { Violent } \\
\text { recidivism }\end{array}$ & $\begin{array}{l}\text { Technical } \\
\text { violations }\end{array}$ \\
\hline & $\mathrm{n}(\%)$ & $\mathrm{n}(\%)$ & $\mathrm{n}(\%)$ & $\mathrm{n}(\%)$ & $\mathrm{n}(\%)$ & $\mathrm{n}(\%)$ & $\mathrm{n}(\%)$ \\
\hline Low & $17(15.18)$ & $5(4.46)$ & $8(7.14)$ & $26(23.21)$ & $46(11.56)$ & $7(1.76)$ & $76(19.10)$ \\
\hline Moderate & $24(21.43)$ & $13(11.61)$ & $18(16.07)$ & $26(23.21)$ & $68(17.09)$ & $15(3.77)$ & $119(29.90)$ \\
\hline Moderate/High & $17(15.18)$ & $7(6.25)$ & $13(11.61)$ & $26(23.21)$ & $21(5.28)$ & $5(1.26)$ & $56(14.07)$ \\
\hline High & $1(0.89)$ & $1(0.89)$ & $1(0.89)$ & $1(0.89)$ & $0(0.00)$ & $0(0.00)$ & $1(0.25)$ \\
\hline
\end{tabular}

Note. General recidivism includes IPV recidivism for IPV offenders only. DRAOR = Dynamic Risk Assessment for Offender Re-Entry; IPV = intimate partner violence.

${ }^{\mathrm{a}} N=112 .{ }^{\mathrm{b}} N=398$ 
Research Question 1c: Do more IPV offenders fall into higher DRAOR risk levels than non-IPV offenders?

Table 9 provides the number and percentage of IPV and non-IPV offenders within each DRAOR risk level. Fewer IPV offenders were classified as Low and Moderate risk, whereas more IPV offenders were classified as Moderate/High and High risk; however, these differences were not large. Further, the ordinal test statistic, $M^{2}$ - the linear trend alternative to the Pearson's chi-squared test of independence-was nonsignificant, $M^{2}(1)=2.34, p=.13$, suggesting that DRAOR risk level is independent of offender typology.

Table 9

IPV and Non-IPV Offenders in Each DRAOR Risk Level (ns and \%)

\begin{tabular}{lcc}
\hline & \multicolumn{1}{c}{ IPV } & Non-IPV \\
\cline { 2 - 3 } DRAOR risk level & $n(\%)$ & $n(\%)$ \\
\hline Low & $42(37.50)$ & $175(43.97)$ \\
Moderate & $40(35.71)$ & $157(39.45)$ \\
Moderate/High & $29(25.89)$ & $65(16.33)$ \\
High & $1(0.89)$ & $1(0.25)$ \\
Total & $112(100.00)$ & $398(100.00)$ \\
\hline
\end{tabular}

Note. DRAOR = Dynamic Risk Assessment for Offender Re-Entry; IPV = intimate partner violence.

\section{Psychometrics}

Research Question 2: Does the DRAOR demonstrate adequate psychometric properties (i.e., reliability and convergent/divergent validity) with IPV offenders?

Internal consistency estimates, as measured by Cronbach's alphas, were acceptable ${ }^{30}$ for the Stable $(\alpha=.83)$ and Protective subscales $(\alpha=.86)$, but below the acceptable level for the Acute subscale $(\alpha=.65)$. Table 10 presents item-total correlations and Cronbach's alphas if items

\footnotetext{
${ }^{30}$ Recall that Cronbach's alphas of .70 (Reynolds \& Livingstone, 2012) or .80 (Clark \& Watson, 1995) and above indicate acceptable internal consistency.
} 
are deleted. Item-total correlations were acceptable ${ }^{31}$ for the Stable (ranging from $r=.49$ to .69) and Protective subscales (ranging from $r=.61$ to .70 ), and generally acceptable for the Acute subscale (ranging from $r=.28$ to .52 ), with only the substance abuse and employment items below the acceptable level. Across all subscales, no improvements to internal consistency would be made if any of the items were deleted.

\footnotetext{
${ }^{31}$ Recall that item-total correlations of .30 and above indicate acceptable internal consistency (Field, 2009).
} 
Table 10

DRAOR Item-Total Correlations and Cronbach's Alphas if Item Deleted for IPV Sample

\begin{tabular}{lcc}
\hline DRAOR item & $\begin{array}{c}\text { Corrected item-total } \\
\text { correlation }\end{array}$ & $\begin{array}{c}\text { Cronbach's } \alpha \text { if item } \\
\text { deleted }\end{array}$ \\
\hline Stable & & .83 \\
Peer Associations & .49 & .79 \\
Attitudes Towards Authority & .69 & .79 \\
Impulse Control & .66 & .82 \\
Problem Solving & .52 & .79 \\
Sense of Entitlement & .65 & .80 \\
Attachment with Others & .61 & .64 \\
Acute & & .57 \\
Substance Abuse & .28 & .62 \\
Anger/Hostility & .52 & .62 \\
Opportunity/Access to Victims & .34 & .64 \\
Negative Mood & .33 & .59 \\
Employment & .29 & .62 \\
Interpersonal Relationships & .45 & .82 \\
Living Situation & .35 & .83 \\
Protective & & .84 \\
Responsive to Advice & .66 & .84 \\
Prosocial Identity & .61 & .83 \\
High Expectations & .64 & .83 \\
Costs/Benefits & .64 & \\
Social Support & .64 & \\
Social Control & &
\end{tabular}

Note. Stable subscale Cronbach's $\alpha=.83$. Acute subscale Cronbach's $\alpha=.65$. Protective subscale Cronbach's $\alpha=.86$. DRAOR $=$ Dynamic Risk Assessment for Offender Re-Entry.

Convergent and divergent validity results are displayed in Table 11. Inter-correlations between subscales were large $\mathrm{e}^{32}$ and in the expected directions, as were the correlations between the DRAOR Total and domains (note that all correlations were statistically significant). The IRA

\footnotetext{
${ }^{32}$ Recall that Correlations of $.10, .30$, and .50 represent small, moderate, and large effect sizes, respectively (Cohen, 1992).
} 
$(M=14.13, S D=5.40)$ produced small, but significant positive correlations with the DRAOR Total, Stable, and Acute measures, however, the correlation between the IRA and the Protective subscale did not reach statistical significance.

Table 11

Correlations Between Risk and Protective Measures for IPV Sample

\begin{tabular}{lccccc}
\hline Risk/protective measure & 1 & 2 & 3 & 4 & 5 \\
\hline 1. DRAOR Stable & - & - & - & - & - \\
2. DRAOR Acute & $.67^{* *}$ & - & - & - & - \\
3. DRAOR Protective & $-.69^{* *}$ & $-.60^{* *}$ & - & - & - \\
4. DRAOR Total & $.89^{* *}$ & $.87^{* *}$ & $-.86^{* *}$ & - & - \\
5. IRA & $.24 * *$ & $.21 *$ & -.10 & $.21 * *$ & - \\
\hline
\end{tabular}

Note. DRAOR = Dynamic Risk Assessment for Offender Re-Entry; IRA = Iowa Risk Assessment. $* p<.05 . * * p<.01$.

\section{Predictive Validity}

Research Question 3a: Do the DRAOR Total and domains accurately predict general recidivism, IPV recidivism, violent recidivism, and technical violations among IPV offenders?

Research Question 3b: Do individual DRAOR items accurately predict IPV recidivism among IPV offenders?

Research Question 3c: Is the IRA an accurate predictor of general recidivism, IPV recidivism, violent recidivism, and technical violations among IPV offenders?

Predictive validity results for IPV offenders across the four community outcomes are presented in Table 12. The DRAOR Acute domain significantly predicted general recidivism $(\mathrm{AUC}=.62$, a small effect $)$, with higher scores associated with an increased likelihood in recidivism. None of the other DRAOR measures, nor the IRA predicted general recidivism. In terms of IPV recidivism, neither the DRAOR Total, domains, nor the IRA produced significant 
AUCs. The DRAOR Total and Stable scores were significantly positively associated with violence (i.e., higher rates of recidivism; $\mathrm{AUCs}=.65$, moderate effects), whereas Protective scores were significantly negatively associated with violence (i.e., lower rates of recidivism; $\mathrm{AUC}=.35$, a moderate effect). The negative relationship between Protective scores and violent recidivism is expected, given that higher scores on the Protective domain reflect lower risk. Neither the Acute subscale, nor the IRA predicted violent recidivism. The DRAOR Total, Stable, and Acute significantly predicted technical violations (AUCs $=.65$, moderate effects), with higher scores associated with an increased likelihood of violating conditions. The DRAOR Protective domain also significantly predicted violations $(\mathrm{AUC}=.38$, a small effect), with higher scores associated with a decreased likelihood of violation conditions. Lastly, higher scores on the IRA were significantly associated with an increased likelihood of violating supervision conditions $(\mathrm{AUC}=.62$, a small effect). 
Table 12

Predictive Validity of the DRAOR and IRA (AUCS) Across Outcomes for IPV Sample

\begin{tabular}{lcccc}
\hline & \multicolumn{4}{c}{ AUC $[95 \% \mathrm{CI}]$} \\
\cline { 2 - 5 } Risk/protective measure & General recidivism & IPV recidivism & Violent recidivism & Technical violations \\
\hline DRAOR Stable & $.60[.49, .70]$ & $.62[.50, .73]$ & $\mathbf{. 6 5}[. \mathbf{5 4}, \mathbf{. 7 5}]$ & $\mathbf{. 6 5}[\mathbf{. 5 4}, \mathbf{. 7 6}]$ \\
DRAOR Acute & $\mathbf{. 6 2}[. \mathbf{5 1}, . \mathbf{7 2}]$ & $.55[.43, .68]$ & $.60[.49, .71]$ & $\mathbf{. 6 5}[.54, .76]$ \\
DRAOR Protective & $.41[.31, .52]$ & $.42[.29, .55]$ & $\mathbf{. 3 5}[. \mathbf{2 4}, \mathbf{. 4 6}]$ & $\mathbf{. 3 8}[.27, . \mathbf{4 9}]$ \\
DRAOR Total & $.61[.50, .71]$ & $.60[.48, .72]$ & $\mathbf{. 6 5}[.55, .76]$ & $\mathbf{. 6 5}[.54, .76]$ \\
IRA & $.52[.42, .63]$ & $.51[.39, .64]$ & $.52[.41, .63]$ & $\mathbf{. 6 2}[.51, .73]$ \\
\hline
\end{tabular}

Note. Significant AUCs are bolded. DRAOR = Dynamic Risk Assessment for Offender Re-Entry; IRA = Iowa Risk Assessment; AUC = area under the curve statistic; $\mathrm{CI}=$ confidence interval. 
AUCs for the accuracy of individual DRAOR items in predicting IPV recidivism are presented in Table 13. Apart from the sense of entitlement item from the Stable domain (AUC $=$ .64 , a moderate effect), none of the items significantly predicted recidivism. In terms of magnitude, AUCs ranged from less than small to moderate for Stable items, and less than small to small for Acute and Protective items.

Table 13

Predictive Validity of DRAOR Items (AUCs) for IPV Recidivism

\begin{tabular}{|c|c|}
\hline DRAOR item & $\mathrm{AUC}[95 \% \mathrm{CI}]$ \\
\hline \multicolumn{2}{|l|}{ Stable } \\
\hline Peer Associations & $.53[.41, .65]$ \\
\hline Attitudes Towards Authority & $.55[.43, .67]$ \\
\hline Impulse Control & $.54[.42, .67]$ \\
\hline Problem Solving & $.54[.41, .67]$ \\
\hline Sense of Entitlement & $.64[.53, .75]$ \\
\hline Attachment with Others & $.60[.49, .71]$ \\
\hline \multicolumn{2}{|l|}{ Acute } \\
\hline Substance Abuse & $.57[.45, .69]$ \\
\hline Anger/Hostility & $.51[.39, .63]$ \\
\hline Opportunity/Access to Victims & $.60[.47, .73]$ \\
\hline Negative Mood & $.53[.41,66]$ \\
\hline Employment & $.51[.39, .64]$ \\
\hline Interpersonal Relationships & $.49[.36, .62]$ \\
\hline Living Situation & $.54[.42, .67]$ \\
\hline \multicolumn{2}{|l|}{ Protective } \\
\hline Responsive to Advice & $.43[.31, .55]$ \\
\hline Prosocial Identity & $.53[.42, .67]$ \\
\hline High Expectations & $.46[.34, .58]$ \\
\hline Costs/Benefits & $.49[.36, .63]$ \\
\hline Social Support & $.39[.26, .52]$ \\
\hline Social Control & $.40[.27, .53]$ \\
\hline
\end{tabular}

Note. Significant AUCs are bolded. AUCs below .50 indicate that higher scores (i.e. more

Protective factors) were associated with lower rates of recidivism. DRAOR $=$ Dynamic Risk 
Assessment for Offender Re-Entry; IPV = intimate partner violence; AUC = area under the curve statistic; $\mathrm{CI}=$ confidence interval.

Research Question 3d: Does the probability of general recidivism, IPV recidivism, violent recidivism, and technical violations increase as IPV and non-IPV offenders move up in DRAOR risk level?

Binary logistic regression analyses were used to determine if the probability of recidivism and technical violations increase as offenders move up in DRAOR risk level, as well as to produce expected recidivism/violation rates for each DRAOR Total score. Results for the overall sample, IPV offenders, and non-IPV offenders are presented in Table 14. All odds ratios were above 1 (effects were less than small in magnitude), indicating that higher DRAOR Total scores were related to higher odds of recidivism and technical violations. DRAOR Total scores significantly predicted general, IPV, and violent recidivism, as well as technical violations for the overall sample. Among IPV offenders, DRAOR Total scores significantly predicted general recidivism, violent recidivism, and technical violations, but not IPV recidivism. Lastly, DRAOR Total scores significantly predicted general recidivism and technical violations among non-IPV offenders, but not violent recidivism. All effect sizes were less than small in magnitude.

Expected recidivism and technical violation probabilities for the overall sample at each DRAOR Total score are depicted in Figures 2 to 5. These figures also delineate outcome estimates for DRAOR risk level cut-offs $($ Low $=-12$ to 2 ; Moderate $=3$ to 9 ; Moderate/High $=$ 10 to $22 ;$ High $=23$ to 26). As shown in Figure 2, general recidivism estimates ranged from $22.86 \%$ to $36.08 \%$ for Low risk offenders, $37.15 \%$ to $43.78 \%$ for Moderate risk offenders, $44.92 \%$ to $58.61 \%$ for Moderate/High risk offenders, and $59.72 \%$ to $62.99 \%$ for High risk offenders. IPV recidivism estimates are illustrated in Figure 3, ranging from $1.56 \%$ to $4.10 \%$ for Low risk offenders, $4.39 \%$ to $6.56 \%$ for Moderate risk offenders, $7.01 \%$ to $15.02 \%$ for 
Moderate/High risk offenders, and $15.95 \%$ to $19.02 \%$ for High risk offenders. Figure 4 presents predicted probabilities for violent recidivism, ranging from $4.07 \%$ to $10.81 \%$ for Low risk offenders, $11.56 \%$ to $17.01 \%$ for Moderate risk offenders, $18.09 \%$ to $35.21 \%$ for Moderate/High risk offenders, and $36.94 \%$ to $42.31 \%$ for High risk offenders. Lastly, as shown in Figure 5, technical violation estimates ranged from $22.48 \%$ to $63.18 \%$ for Low risk offenders, $66.08 \%$ to $80.67 \%$ for Moderate risk offenders, $82.58 \%$ to $95.61 \%$ for Moderate/High risk offenders, and 96.11\% to $97.31 \%$ for High risk offenders. Expected recidivism and technical violation probabilities for DRAOR risk level cut-offs among IPV and non-IPV offenders are listed in Table 15. All outcome estimates increased as IPV and non-IPV offenders moved up in DRAOR risk level. 
Table 14

Logistic Regression Analyses for DRAOR Total Scores Predicting Community Outcomes Among Overall Sample, IPV, and Non-IPV Offenders

\begin{tabular}{lcccccccc}
\hline & & & & & & & \multicolumn{2}{c}{$95 \%$ CI for OR } \\
\cline { 6 - 9 } Outcome & $B$ & $S E$ & Wald & $d f$ & $p$ & OR & Lower & Upper \\
\hline Overall sample & & & & & & & & \\
$\quad$ General recidivism & .046 & .013 & 13.255 & 1 & .000 & 1.048 & 1.022 & 1.074 \\
$\quad$ IPV recidivism & .071 & .028 & 6.371 & 1 & .012 & 1.074 & 1.016 & 1.135 \\
$\quad$ Violent recidivism & .075 & .019 & 15.801 & 1 & .000 & 1.078 & 1.039 & 1.118 \\
$\quad$ Technical violations & .127 & .015 & 66.931 & 1 & .000 & 1.135 & 1.101 & 1.170 \\
IPV offenders & & & & & & & & \\
$\quad$ General recidivism & .062 & .027 & 5.055 & 1 & .025 & 1.064 & 1.008 & 1.122 \\
$\quad$ IPV recidivism & .044 & .031 & 1.953 & 1 & .162 & 1.045 & .983 & 1.110 \\
$\quad$ Violent recidivism & .075 & .029 & 6.596 & 1 & .010 & 1.078 & 1.018 & 1.142 \\
$\quad$ Technical violations & .085 & .031 & 7.333 & 1 & .007 & 1.089 & 1.024 & 1.158 \\
Non-IPV offenders & & & & & & & & \\
$\quad$ General recidivism & .036 & .015 & 6.038 & 1 & .014 & 1.037 & 1.007 & 1.067 \\
$\quad$ Violent recidivism & .054 & .028 & 3.706 & 1 & .054 & 1.055 & .999 & 1.114 \\
$\quad$ Technical violations & .138 & .018 & 59.140 & 1 & .000 & 1.148 & 1.108 & 1.189 \\
\hline
\end{tabular}

Note. $\mathrm{IPV}=$ intimate partner violence; $\mathrm{OR}=$ odds ratio. 


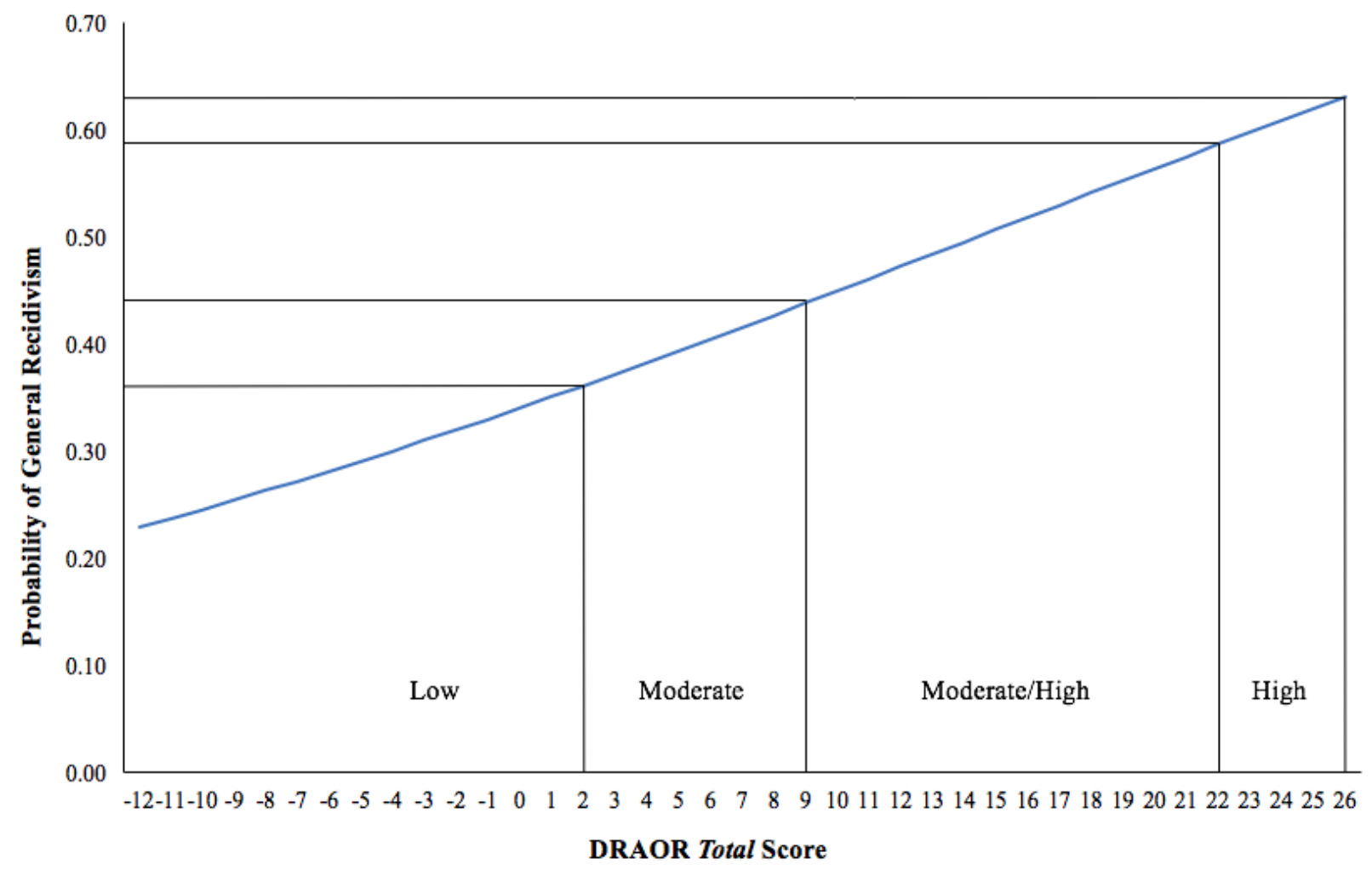

Figure 2. Probability of general recidivism across DRAOR Total scores and risk level for the overall sample. 


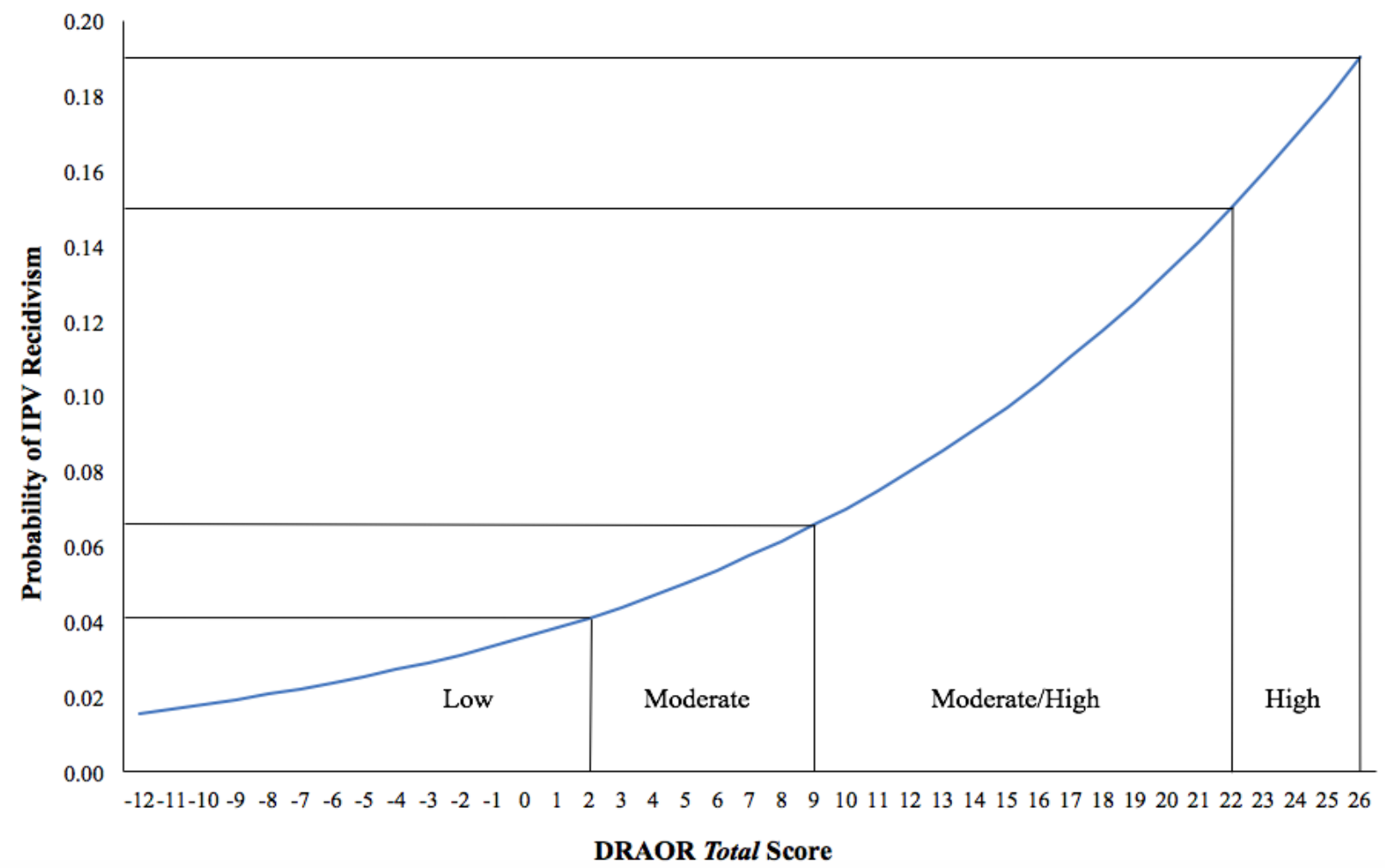

Figure 3. Probability of IPV recidivism across DRAOR Total scores and risk level for the overall sample. 


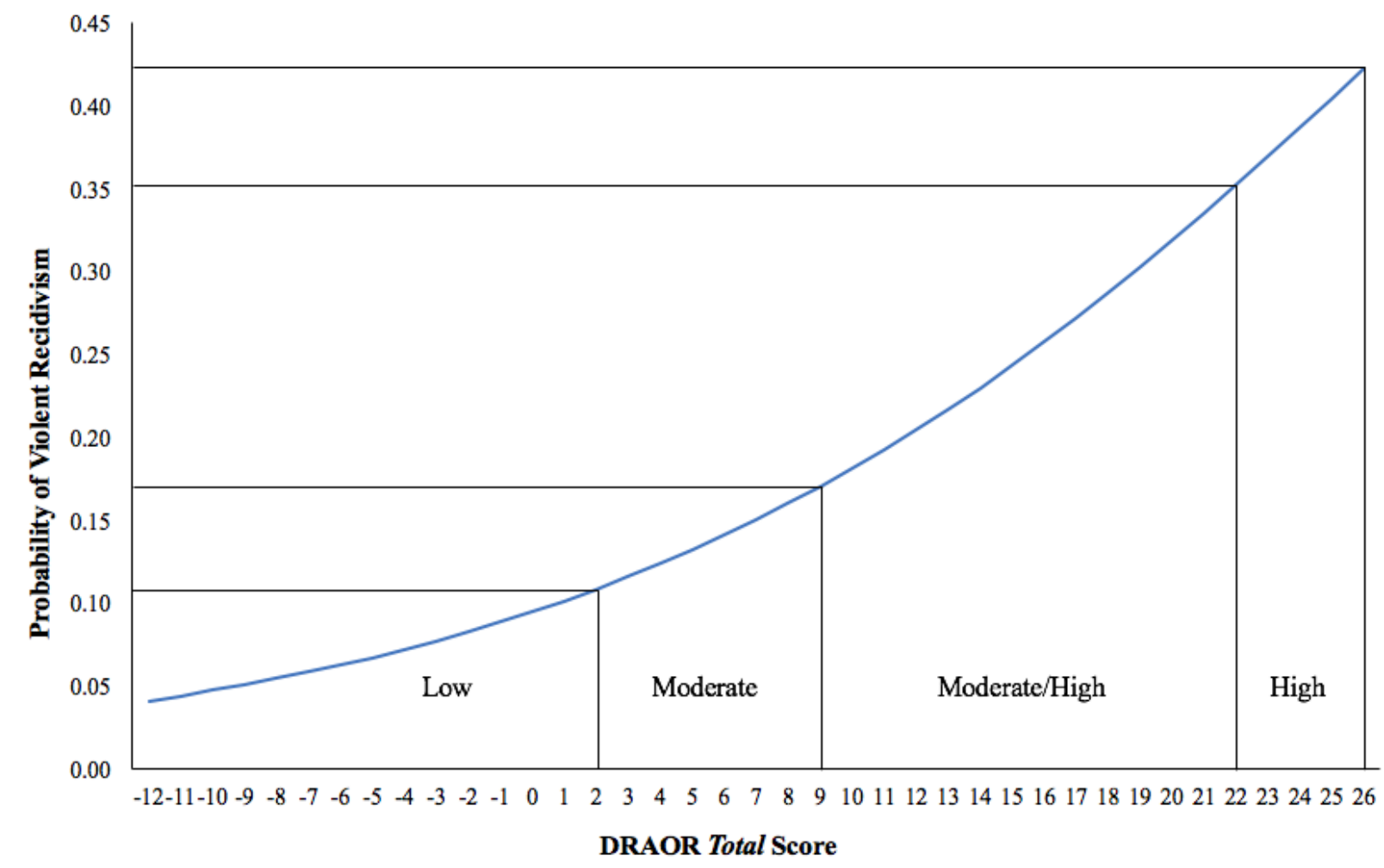

Figure 4. Probability of violent recidivism across DRAOR Total scores and risk level for the overall sample. 


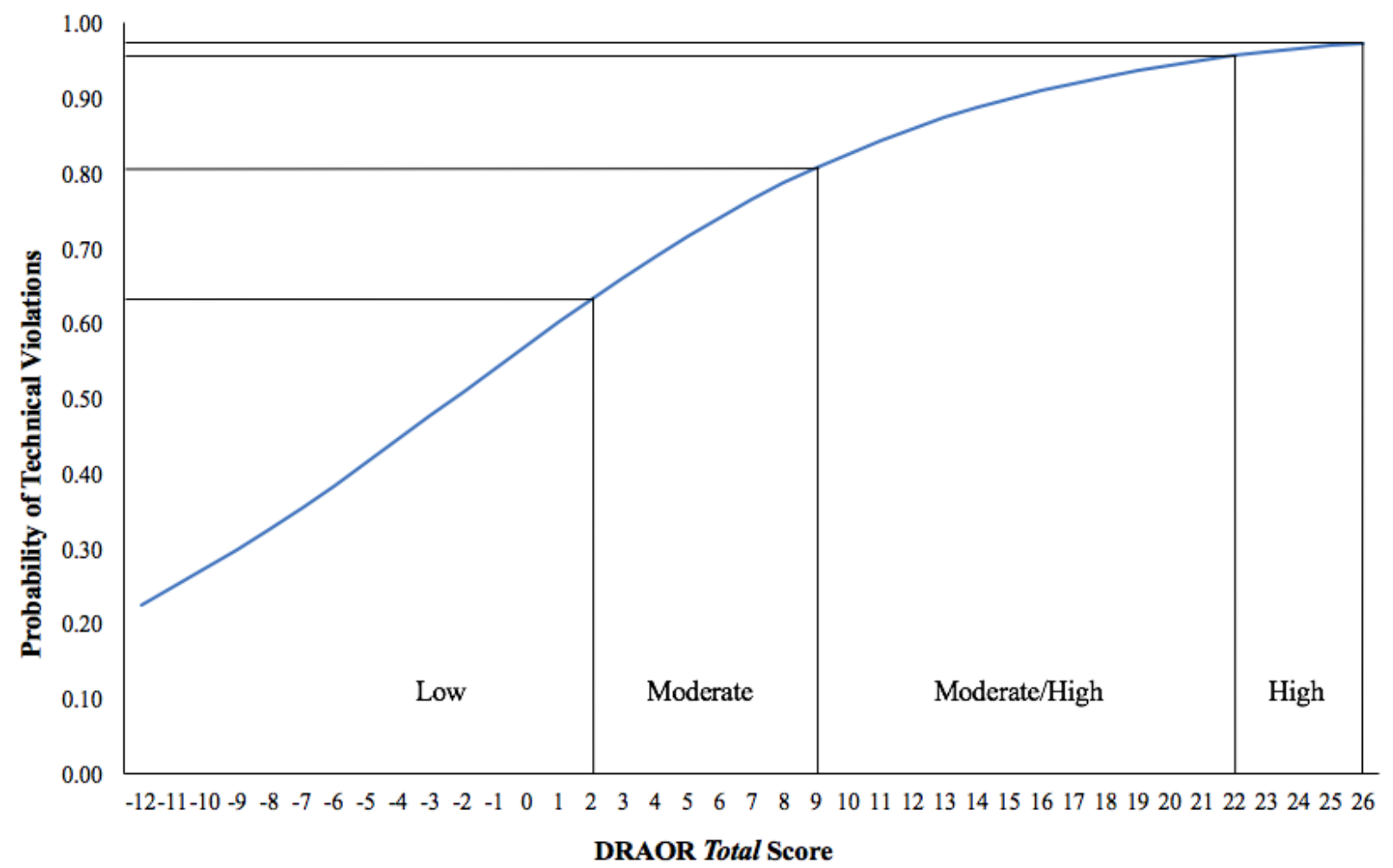

Figure 5. Probability of technical violations across DRAOR Total scores and risk level for the overall sample. 
Table 15

Likelihood of Community Outcomes for DRAOR Risk Level Cut-offs Among IPV and Non-IPV Offenders

\begin{tabular}{|c|c|c|c|c|c|c|c|c|}
\hline \multirow[b]{2}{*}{ DRAOR risk level } & \multicolumn{2}{|c|}{ General recidivism } & \multicolumn{2}{|c|}{ IPV recidivism } & \multicolumn{2}{|c|}{ Violent recidivism } & \multicolumn{2}{|c|}{ Technical violations } \\
\hline & Lower \% & Upper \% & Lower $\%$ & Upper \% & Lower \% & Upper \% & Lower \% & Upper \% \\
\hline \multicolumn{9}{|l|}{ IPV } \\
\hline Low & 27.69 & 47.70 & 12.09 & 20.29 & 12.69 & 29.34 & 37.17 & 66.04 \\
\hline Moderate & 49.25 & 58.47 & 21.02 & 25.73 & 30.92 & 41.24 & 67.92 & 77.90 \\
\hline Moderate/High & 59.96 & 75.91 & 26.58 & 38.04 & 43.07 & 65.04 & 79.33 & 91.41 \\
\hline High & 77.03 & 80.15 & 39.08 & 42.26 & 66.73 & 71.52 & 92.06 & 93.73 \\
\hline \multicolumn{9}{|l|}{ Non-IPV } \\
\hline Low & 22.83 & 32.87 & & & 2.95 & 6.08 & 22.86 & 36.08 \\
\hline Moderate & 33.67 & 38.65 & & & 6.39 & 8.63 & 37.15 & 43.78 \\
\hline Moderate/High & 39.51 & 50.15 & & & 9.06 & 16.00 & 44.92 & 58.61 \\
\hline High & 51.05 & 53.74 & & & 16.74 & 19.12 & 59.72 & 62.99 \\
\hline
\end{tabular}

Note. DRAOR = Dynamic Risk Assessment for Offender Re-Entry; IPV = intimate partner violence; Lower/Upper = the lower and upper DRAOR risk level cut-offs (Low $=-12$ to 2 ; Moderate $=>2$ to 9 ; Moderate/High $=>9$ to 22; High $=>22$ to 26). 


\section{Incremental Predictive Validity}

Research Question 4: Do the DRAOR Total, Stable, Acute, and Protective subscales improve the prediction of time to general recidivism, IPV recidivism, violent recidivism, and technical violations among IPV offenders beyond static risk (i.e., the IRA)? A series of univariate Cox regression survival analyses were used to determine if the DRAOR Total, DRAOR domains, and the IRA predict time to each community outcome. Results are shown in Table 16. For any recidivism, only the DRAOR Total and Acute were significantly related to survival time, with recidivism increasing by $4.1 \%$ for each unit increase in the Total score and $11.5 \%$ for each unit increase the Acute score. None of the static or dynamic measures significantly predicted time to IPV recidivism. DRAOR Total, Stable, and Protective scores significantly predicted time to violent recidivism, but Acute and IRA scores did not. Violent recidivism increased by $5.5 \%$ for each unit increase in the Total score and $14.6 \%$ for each unit increase in the Stable score, while each unit increase in the Protective score resulted in a $12.4 \%$ decrease in violent recidivism. Lastly, all DRAOR measures significantly predicted time to technical violations, but the IRA did not. Each unit increase in DRAOR Total, Stable, and Acute scores resulted in a $6.1 \%, 14.2 \%$, and $13.9 \%$ increase in technical violations, respectively, and technical violations decreased by $11.7 \%$ for each unit increase in the Protective score. Due to the null findings for the IRA predicting time to community outcomes, the incremental predictive validity of the DRAOR Total and domains over static risk was not assessed. 
Table 16

Univariate Cox Regression Survival Analyses Predicting Time to Outcomes Among IPV Offenders

\begin{tabular}{|c|c|c|c|c|c|c|c|c|}
\hline \multirow[b]{2}{*}{ Variable } & \multirow[b]{2}{*}{$B$} & \multirow[b]{2}{*}{$S E$} & \multirow[b]{2}{*}{ Wald } & \multirow[b]{2}{*}{$d f$} & \multirow[b]{2}{*}{$p$} & \multirow[b]{2}{*}{$\mathrm{HR}$} & \multicolumn{2}{|c|}{$95 \% \mathrm{CI}$ for $\mathrm{HR}$} \\
\hline & & & & & & & Lower & Upper \\
\hline \multicolumn{9}{|c|}{ Any recidivism } \\
\hline \multicolumn{9}{|c|}{ DRAOR } \\
\hline Stable & .088 & .046 & 3.611 & 1 & .057 & 1.092 & 0.997 & 1.195 \\
\hline Acute & .109 & .043 & 6.353 & 1 & .012 & 1.115 & 1.025 & 1.214 \\
\hline Protective & -.071 & .049 & 2.134 & 1 & .144 & 0.932 & 0.847 & 1.025 \\
\hline Total & .040 & .018 & 5.148 & 1 & .023 & 1.041 & 1.005 & 1.077 \\
\hline IRA & .008 & .025 & 0.103 & 1 & .748 & 1.008 & 0.960 & 1.059 \\
\hline \multicolumn{9}{|c|}{ IPV recidivism } \\
\hline \multicolumn{9}{|l|}{ DRAOR } \\
\hline Stable & .094 & .068 & 1.885 & 1 & .170 & 1.098 & 0.961 & 1.256 \\
\hline Acute & .063 & .066 & 0.935 & 1 & .334 & 1.065 & 0.937 & 1.211 \\
\hline Protective & -.081 & .073 & 1.244 & 1 & .265 & 0.922 & 0.799 & 1.064 \\
\hline Total & .034 & .026 & 1.713 & 1 & .191 & 1.035 & 0.983 & 1.089 \\
\hline IRA & .029 & .041 & 0.517 & 1 & .472 & 1.030 & 0.951 & 1.115 \\
\hline \multicolumn{9}{|c|}{ Violent recidivism } \\
\hline \multicolumn{9}{|c|}{ DRAOR } \\
\hline Stable & .136 & .056 & 5.999 & 1 & .014 & 1.146 & 1.028 & 1.278 \\
\hline Acute & .103 & .053 & 3.744 & 1 & .053 & 1.109 & 0.999 & 1.231 \\
\hline Protective & -.132 & .059 & 5.020 & 1 & .025 & 0.876 & 0.780 & 0.984 \\
\hline Total & .054 & .021 & 6.325 & 1 & .012 & 1.055 & 1.012 & 1.100 \\
\hline IRA & .023 & .031 & 0.547 & 1 & .460 & 1.023 & 0.963 & 1.088 \\
\hline \multicolumn{9}{|c|}{ Technical violations } \\
\hline \multicolumn{9}{|l|}{ DRAOR } \\
\hline Stable & .133 & .040 & 10.995 & 1 & .001 & 1.142 & 1.056 & 1.236 \\
\hline Acute & .130 & .040 & 10.500 & 1 & .001 & 1.139 & 1.053 & 1.233 \\
\hline Protective & -.124 & .045 & 7.708 & 1 & .005 & 0.883 & 0.809 & 0.964 \\
\hline Total & .059 & .016 & 13.157 & 1 & .000 & 1.061 & 1.027 & 1.095 \\
\hline IRA & .033 & .026 & 1.657 & 1 & .198 & 1.034 & 0.983 & 1.088 \\
\hline
\end{tabular}

Note. DRAOR = Dynamic Risk Assessment for Offender Re-Entry; IPV = intimate partner violence; HR = hazard ratio; CI = confidence interval. 


\section{Discussion}

The current study examined the predictive validity of the DRAOR for violence in intimate partner relationships. This research is the first to investigate whether the DRAOR accurately predicts this type of violence, providing information on how broadly the scale ought to be applied. Although the DRAOR did not appear to predict IPV, it was useful in other contexts. Several findings also helped advance theory and inform risk/case management practices. A summary of these results is provided below.

\section{Differences Between IPV and Non-IPV Offenders}

Preliminary analyses revealed that IPV offenders scored significantly higher than nonIPV offenders on the following items: attachment with others, substance abuse, anger/hostility, opportunity/access to victims, and interpersonal relationships. This partially supports Hypothesis 1a and is consistent with previous research on areas that are particularly problematic for IPV offenders (e.g., Almond et al., 2017; Bennett Cattaneo \& Goodman, 2005; Birkley \& Eckhardt, 2015; Hilton \& Harris, 2005; LaMotte et al., 2018; Norlander \& Eckhardt, 2005). One caveat to these findings, however, is that effect sizes were generally small in magnitude (except for the anger/hostility item). Further, the lower CI limits for attachment with others and substance abuse were .51 , meaning the effects only just reached statistical significance. Caution is therefore warranted when interpreting these results.

In addition to their higher item scores, IPV offenders also received higher Acute and Total scores than non-IPV offenders, indicating they pose greater acute and overall risk. Contrary to Hypothesis 1a, IPV and non-IPV offenders did not differ on the Stable item impulse control, the Acute items negative mood or employment, or the Protective item social support. Recent research found that negative affect predicts IPV perpetration among male university 
students (Shorey et al., 2015); however, few studies examine this relationship in offender samples and those that do are fairly dated (e.g., Jacobson et al., 1996; Palmer et al., 1992). The link between unemployment and IPV recidivism has been more commonly studied and supported (e.g., Caetano, 2005; Edwards, 2015), but research is still mixed (Bennett Cattaneo \& Goodman, 2005). Despite the null findings in the current study, the literature suggests that negative mood and unemployment may be important treatment targets for IPV offenders. Finally, while research on social support as a protective factor for IPV recidivism is lacking, this relationship might be worth exploring given the link between social support and violence desistance (Ulrich \& Coid, 2011).

IPV offenders had higher base rates for general recidivism, violent recidivism, and technical violations than non-IPV offenders. This supports Hypothesis $1 \mathrm{~b}$ and is not surprising given their higher scores on the DRAOR Total. It is important to note, however, that base rates might be slightly inflated for IPV offenders, as 17 individuals were categorized into this group because they committed an IPV recidivistic offence (i.e., 17 individuals had no previous or index IPV offence, but were classified as IPV offenders because of their repeat offence). A detailed examination of base rates across DRAOR risk levels revealed that more Low, Moderate, Moderate/High, and High risk IPV offenders committed a new general and violent offence than non-IPV offenders. The same pattern was found for technical violations, except for the Moderate group; more non-IPV offenders in this risk bin violated a supervision order than IPV offenders. Although IPV offenders scored significantly higher on the DRAOR Total, they were not more likely than non-IPV offenders to be placed in higher versus lower DRAOR risk bins, raising concerns regarding current DRAOR cut-offs. 
The overall performance of DRAOR risk levels was not optimal. Recidivism rates should increase steadily from Low to High risk bins, but base rates in the current sample generally increased from Low to Moderate and then decreased from Moderate to High for both offender typologies. One possible explanation is that the sample was too small to obtain reliable base rates across risk levels (i.e., few offenders were rated as Moderate/High and High risk). The one High risk IPV offender, for example, committed a general, IPV, and violent offence (all likely the same offence), and violated a condition of his release, but this still only resulted in base rates of less than $1 \%$. A second explanation is that DRAOR risk bins do not capture offenders' true level of risk. Cut-off scores need to be revised if this is the case.

The above findings advance theory on IPV and inform the management of IPV offenders. For example, different item scores across offender typologies provide partial evidence for the ecological model for violence perpetration. Recall that this model uses individual, relationship, community, and societal factors to explain violence in intimate partner relationships (Dahlberg \& Krug, 2002) and that DRAOR items map onto the first three of these factors. IPV offenders scored significantly higher on three individual variables (attachment with others, ${ }^{33}$ substance abuse, anger/hostility) and three relationship variables (attachment with others, ${ }^{34}$ opportunity/access to victims, interpersonal relationships). This supports the theory that IPV is influenced by both individual and contextual factors. Beyond contributing to theory, the differences found in domain and item scores across typologies also inform intervention strategies. IPV offenders are likely to benefit from supervision that targets Acute risk and treatment needs that may be especially important for these individuals are attachment with

\footnotetext{
${ }^{33}$ Although it may appear that attachment with others is a relationship-only variable, it partly focuses on a lack of concern for others - an individual variable — that results in social disconnection or problematic interpersonal attachments, thus it also qualifies as an individual variable.

${ }^{34}$ In addition to being classified as an individual variable, attachment with others qualifies as a relationship variable as well because it recognizes the nature of relationships with others, such as short-term or superficial relationships.
} 
others, substance abuse, anger/hostility, opportunity/access to victims, and interpersonal relationships.

\section{Psychometrics}

Reliability analyses were acceptable for the Stable and Protective domains, but below the acceptable level for the Acute domain. The same findings were reported in previous research as well (Chadwick, 2014; Hanby, 2013). As the DRAOR domains were not derived from factor analysis and do not imply pure constructs, these results are not unexpected. Acute substance abuse and employment items were not strongly related to the Acute domain score in the current study or in previous research (Chadwick, 2014; Hanby, 2013), but removing these items did not improve internal consistency estimates. Thus, substance abuse and employment are likely valid measures of destabilizers and lifestyle stressors - case management constructs reflected in the Acute domain. Further, Acute items reflect instability, but individual cases will not experience all these factors at all times. Hence item-total correlations for this domain are not expected to be high. Moreover, as a case planning tool reflecting heterogeneous domains, it is more important the domains have predictive validity than internal consistency (Helmus \& Babchishin, 2017).

The current study provided support for the convergent and divergent validity of the DRAOR. Correlations between the DRAOR Total and domains were in the expected directions, as were the inter-correlations between subscales. Correlations involving the IRA were also in the expected direction, but the association between the IRA and Protective domain was nonsignificant. These findings are generally consistent with previous research showing that (1) the Stable subscale acts similarly to a risk-need scale and targets traditional criminogenic needs, (2) the Acute subscale addresses risk factors for supervision failure and can be used to refine case planning and risk management strategies, and (3) the Protective subscale measures factors that 
operate differently than risk indicators and can be used to develop a more balanced, positive approach to risk assessment. Taken together, the reliability and convergent/divergent validity results supported Hypothesis 2.

\section{Predictive Validity}

None of the DRAOR measures predicted IPV recidivism among IPV offenders and only the Acute domain predicted general recidivism (though the lower CI limit was .51, meaning this effect merely met statistical significance). Results were more promising for the other outcomes, with the Total score and all but the Acute domain predicting violent recidivism, and the DRAOR Total score and all domains predicting technical violations (note that the Protective domain predicted desistance from these outcomes). These findings were partially consistent with Hypothesis 3a. The IRA did not predict any of the recidivism outcomes, but it did predict technical violations.

The DRAOR Total predicted violent recidivism and technical violations with moderate strength, which is acceptable for an instrument intended to be a case management tool. For instance, there are some scales with AUCs comparable to the DRAOR's that are used as risk tools and as case management tools, such as the Ohio Risk Assessment System (ORAS; Latessa, Lemke, Makarios, Smith, \& Lowenkamp, 2010). AUCs for predicting re-arrest during a one-year follow-up period ranged from moderate to large across versions ${ }^{35}$ (AUCs $=.65$ to .71 ; Latessa et al., 2010). These effect sizes have supported the use of the ORAS as a risk assessment instrument, therefore, the use of the DRAOR as a risk/case management tool for violence and technical violations still seems warranted despite the lower AUCs.

\footnotetext{
${ }^{35}$ There are six ORAS versions: Pretrial Assessment Tool, Community Supervision Screening Tool, Community Supervision Tool, Prison Screening Tool, Prison Intake Tool, Re-Entry Tool (Latessa et al., 2010).
} 
A more detailed exploration of the DRAOR's predictive validity for IPV recidivism showed that only the sense of entitlement item in the Stable domain was a significant predictor. It is important to note that this finding is consistent with what would be expected by chance. Specifically, the Type 1 error rate is .05 , which means that five out of every 100 analyses (i.e., roughly 1 in 20) will be significant due to chance. The one DRAOR item (out of 19) that significantly predicted IPV recidivism will therefore not be interpreted as meaningful. Hypothesis $3 \mathrm{~b}$ was not substantiated given the null findings for this research question.

The DRAOR appears to be particularly useful for predicting technical violations among IPV offenders, but not for predicting general or IPV recidivism. The DRAOR was also an accurate predictor of violent recidivism among IPV offenders, but unexpectedly, the Acute domain did not emerge as a significant predictor in this context. This is surprising because many of the Acute items are identified in the IPV literature as significant predictors of partner abuse. Given that all IPV offences are also classified as violent offences, the Acute items should theoretically have had the greatest predictive power for violent recidivism. However, this domain was the only one that failed to predict repeat violence. Certain Acute items may therefore be important predictors of violence in intimate partner relationships, but not violence in nonintimate partner relationships. This hypothesis is unlikely, though, as anger/hostility, opportunity/access to victims, and negative mood (risk factors identified in the literature as IPV correlates) predicted violence against non-intimate partners in a study on 4,116 violent offenders (Lowenkamp, Johnson, Trevino, \& Serin, 2016). A more plausible explanation for these null findings is that there was insufficient statistical power.

Logistic regression was also used to determine if the probability of recidivism and technical violations increased as IPV and non-IPV offenders (both together and separately) 
moved up in DRAOR risk level. These results provided partial support for Hypothesis 3d. Total scores significantly predicted general recidivism, violent recidivism, and technical violations among IPV offenders, but they did not predict IPV recidivism. For non-IPV offenders, DRAOR Total scores significantly predicted general recidivism and technical violations, but not violent recidivism. Lastly, DRAOR Total scores significantly predicted all outcomes for the overall sample. All effect sizes were less than small in magnitude. A possible explanation for the two null findings above is that this study is underpowered. Specifically, 100 events (recidivism) and 100 non-events (no recidivism) are recommended for adequate statistical power in binary logistic regression (Vergouwe et al., 2005). There were only 26 IPV offenders who committed an IPV recidivism offence, however, and 86 who did not. Similarly, 27 non-IPV offenders recidivated violently and 371 did not. Vergouwe and colleagues' (2005) recommendation to achieve adequate statistical power would have been met with a larger sample.

Expected recidivism and violation rates for DRAOR Total scores were also calculated for each offender typology and the overall sample. The goal of these analyses was to provide more intuitive empirical information on how DRAOR risk levels are performing. Expected recidivism rates increased from Low to High risk for both offender typologies and across outcomes, but they varied quite a bit from one group to the next and for different outcomes. For example, a maximum of $47 \%$ of Low risk IPV offenders were expected to commit a new general offence, $20 \%$ were expected to commit a new IPV offence, $29 \%$ a new violent offence, and $66 \%$ a technical violation. Low risk non-IPV offenders had a 33\% chance of general recidivism, a $6 \%$ chance of violent recidivism, and a $36 \%$ chance of a supervision violation (at the upper end of the risk bin). At the other extreme, High risk IPV offenders' maximum likelihood of general recidivism, IPV recidivism, violent recidivism, and technical violations was $80 \%, 42 \%, 72 \%$, 
and 93\%, respectively. The maximum likelihood of general recidivism, violent recidivism, and technical violations for non-IPV offenders was 54\%, 19\%, and 63\%, respectively.

This calls into question what it means for an offender to be deemed low or high risk to reoffend. Consider the following scenario: Using DRAOR Total scores, parole officers are asked to determine supervision level and frequency of contact for an IPV offender and a non-IPV offender. Both individuals are said to be high risk, have similar criminal histories and treatment needs, and are the same age. If these individuals were rated as high risk based only on their DRAOR score, the IPV offender would have an $80 \%$ chance of general recidivism and a $72 \%$ chance of violent recidivism (at the upper end of the risk bin). The non-IPV offender, on the other hand, would have a 54\% chance of general recidivism and a $19 \%$ chance of violent recidivism. Evidently, the rating of "High risk" has two very different meanings for these individuals; inclusion of a static IPV risk measure might greatly improve decision accuracy. This suggests that DRAOR risk levels do not have the same implications for all offender types and community outcomes. Specialized risk levels with better calibration may be more appropriate in these contexts. The same conclusion can be drawn for the DRAOR risk levels when applied to representative samples of offenders. For instance, the maximum likelihood of general recidivism, IPV recidivism, violent recidivism, and technical violations for Low risk offenders in the overall sample was $36 \%, 4 \%, 10 \%$, and $63 \%$, respectively. Among High risk offenders, the greatest chances for these same outcomes were $63 \%, 19 \%, 42 \%$, and $97 \%$, respectively. DRAOR risk levels that are tailored to different outcomes would therefore be required for the scale to be used as a risk tool across diverse offence types. 


\section{Incremental Predictive Validity}

The aim of this research question was to determine whether any of the DRAOR measures improved the predictive accuracy of the IRA, a static risk measure, when predicting time to recidivism and technical violations. The proposed analytic strategy had been to carry out univariate Cox regression survival analyses and then conduct multivariate analyses to assess incremental predictive validity. Univariate analyses precluded the examination of incremental predictive validity, however, as the IRA did not predict time to any of the community outcomes. Examining whether DRAOR measures improve the predictive accuracy of a non-predictive scale would not have yielded meaningful information. For this reason, none of the variables were examined at the multivariate level.

\section{Strengths and Limitations}

One important strength of the current study is that all supervision officers received formal training on the DRAOR, giving confidence that the scale was implemented with fidelity. This is important because poor implementation fidelity has been shown to reduce predictive accuracy (Chadwick, 2014; Flores et al., 2006). The use of field data is a second strength in the methodology of this research; field studies have high ecological validity, meaning that results can be generalized to real-life settings. Two limitations that need to be acknowledged in light of these strengths are (1) implementation fidelity was not formally assessed and (2) the utility of assessment tools is often lower in field studies compared to lab studies (Edens \& Boccaccini, 2017).

Field research in the context of Edens and Boccaccini's (2017) review is defined as the collection of assessment data for "the purpose of informing clinical, forensic, or correctional decision-making in regards to the examinee" (p. 600). This could include decisions like release 
from incarceration or - a primary case management strategy that DRAOR assessments are used for-screening offenders into/out of treatment services (Edens \& Boccaccini, 2017). In lab studies, on the other hand, data is collected solely for research purposes and is not used to inform real-world decisions (Edens \& Boccaccini, 2017). Given that several studies have typically found weaker performance for assessment instruments in field versus lab settings (see Edens \& Boccaccini, 2017, for an overview of this research), the generally low predictive accuracy of the DRAOR in the current field study is not surprising.

A final weakness related to the implementation of the DRAOR concerns supervision quality. Effective supervision can both improve and diminish predictive accuracy. Improved accuracy can be achieved by increasing client-openness and, consequently, the accuracy of item ratings. More accurate ratings would strengthen the potential association between DRAOR scores and recidivism. Conversely, reducing offender risk would decrease the potential association between high DRAOR scores and recidivism. The effects of supervision quality on predictive accuracy can be explored by measuring officers' characteristics and skills as they relate to CCP. There is currently no research in this area, however, which represents a limitation of this study and previous DRAOR works.

The unknown inter-rater reliability of the DRAOR reflects an additional shortcoming of the current and previous studies, as the scale's inter-rater reliability has yet to be examined. The small sample sizes of 112 IPV and 398 non-IPV offenders also resulted in low statistical power for some analyses. Vergouwe and colleagues' (2005) recommendation to achieve adequate statistical power in binary logistic regression likely applies to AUCs as well, for example (L. M. Helmus, personal communication, January 25, 2018). Several outcomes for IPV and non-IPV offenders had less than 100 recidivists and 100 non-recidivists, which could explain some of the 
null findings for predictive validity analyses. Further, the sample was limited to male offenders in the $5^{\text {th }}$ district of Iowa, potentially reducing the generalizability of results to those outside this demographic. A final limitation to the dataset is the missing sexual orientation and victim information. It is likely that offenders were heterosexual and that their victims were adults, however, it is possible that same-sex IPV acts and child abuse were captured in the dataset.

Beyond limitations related to the DRAOR and the sample used in this research, some important measurement issues should be acknowledged. Firstly, cases were identified as IPV offenders if they had any IPV history, index, or recidivism offence. This may have led to the underrepresentation of IPV offenders if any of their IPV offences were undetected. While this measurement issue is inherent to all risk assessment research, it is a particularly noteworthy limitation of research on partner violence. A recent survey found that less than 1 in $5(19 \%)$ Canadians reported their spousal violence victimization to the police, for example (Burczycka \& Ibrahim, 2016). A second measurement issue is the use of initial versus proximal DRAOR scores. Dynamic risk and protective factors show changes in risk across time. Scores that are proximal in time to recidivism should therefore be more closely related to repeat offences than distal scores. Lloyd's (2015) research supported this notion, which could account for the generally non-significant predictive validity findings in the present study.

Lastly, there is little validation information for the IRA, the static risk measure used in this thesis. Serin and colleagues (2018) found the scale to predict technical violations and criminal recidivism, but it demonstrated generally poor predictive accuracy in the current study. It is important to note, however, that the IRA is no longer used in practice and has been replaced with the Iowa Violence and Victimization Instrument (IVVI). The IVVI is a nine-item ${ }^{36}$ actuarial $^{2}$

\footnotetext{
${ }^{36}$ IVVI items include (1) active offences, (2) number of counts of current property offence, (3) ever convicted of murder/manslaughter, robbery, or theft from a person, (4) number of prior counts for violent crimes within the last
} 
instrument comprised of Violence and Victimization subscales. The Violence subscale is designed to predict violent offenses, whereas the Victimization subscale is designed to predict violent and property offenses. Prell and colleagues investigated the predictive validity of the IVVI using a sample of 1,961 males on probation or parole in Iowa for diverse offences (Prell, Vitacco, \& Zavondy, 2016). The five outcomes examined in this study related to drug, property, violent, victimization (violent and property), and any offences. The Violence subscale significantly predicted all outcomes, with small effect sizes for drug and any offences (AUCs $=.56$ and .63 , respectively), moderate effect sizes for property and victimization offences (AUCs =.64 and .69, respectively) and a large effect size for violent offences (AUC $=.71)$. The Victimization subscale also significantly predicted all offences; effect sizes were less than small for drug offences (AUC $=.54)$, moderate for violent, victimization, and any offences $(\mathrm{AUCs}=.70, .70$, and .65 , respectively), and large for property offences $(\mathrm{AUC}=.71)$.

\section{Conclusions and Recommendations}

The DRAOR demonstrated good predictive validity for violent recidivism and technical violations among IPV offenders, but it failed to predict general recidivism or repeat partner violence in this demographic. Acute risk factors that emerged as important treatment targets for IPV offenders were attachment with others, substance abuse, anger/hostility, opportunity/access to victims, and interpersonal relationships. Supervision officers should also focus their attention on IPV offenders' Acute needs in general; these destabilizers and lifestyle stressors were particularly problematic for IPV versus non-IPV offenders. Despite the relatively poor predictive validity results, the current study tentatively supports the use of the DRAOR as a case

\footnotetext{
10 years, (5) prior convictions within the last 10 years, (6) prior conviction for violent crime in the last five years, (7) released from prison or juvenile commitment in the last five years, (8) security threat group membership, and (9) current age. Both the Violence and Victimization subscales assess all nine items, but each subscale has a unique scoring procedure.
} 
management tool for IPV offenders in the absence of a more specialized instrument, but an IPVspecific measure is recommended (Serin \& Lowenkamp, 2015).

Two of the most commonly used and validated IPV-specific risk tools - the ODARA and the DVSI-focus on static risk factors (e.g., prior domestic assault, prior treatment for domestic violence), thus cannot be used for ongoing case management purposes. Another common risk tool for IPV that demonstrates good predictive properties is the SARA. This scale includes both static (e.g., past assault of family members) and dynamic risk factors (e.g., recent relationship problems), but the focus is on the former. The IPV history items also appear to predict recidivism more accurately than the dynamic risk items (Gray, 2012), hence the SARA is not particularly useful for case management purposes either. Therefore, while IPV-specific risk instruments such as the ODARA, DVSI, and SARA are preferable to general risk tools (e.g., the DRAOR) when predicting partner violence, they have limited utility in the ongoing management of offenders. Supervision officers can use the DRAOR to assist with the management of IPV offenders until a more specialized case management tool is developed.

Future research should focus on the development of an IPV-specific case management tool to improve risk reduction strategies among partner-violent offenders. Concurrently, given the low statistical power in the current study, the predictive validity of the DRAOR for IPV recidivism should be examined with a larger sample. This sample should include female offenders to allow for comparisons across gender. Sexual orientation information should also be collected to explore differences between opposite and same-sex couples. Previous research on the DRAOR examined differences in predictive accuracy for Māori and non-Māori offenders and found comparable effect sizes across groups (Hanby, 2013). It is unclear how race influenced the results in the current study, but given that almost half of the IPV sample was non-Caucasian, this 
is an important area for future research. Culture-specific items may be needed to accurately predict repeat partner violence among perpetrators of difference races.

The use of a validated static risk scale would facilitate incremental predictive validity analyses, potentially supporting the integration of the DRAOR into current risk assessment practices for IPV offenders. This would improve our ability to predict recidivism and refine case management strategies if the DRAOR predicts IPV recidivism over and above static risk. Implementation issues that should be addressed include the examination of implementation fidelity and supervision quality. These factors have influenced predictive accuracy in previous research, but have not yet been examined for the DRAOR. Finally, research on the inter-rater reliability of the DRAOR — whether with IPV offenders or a more representative sample — is required to determine if its dynamic risk and protective factors can be reliably assessed across evaluators.

Evaluating the predictive accuracy of risk tools involves the examination of discrimination properties, like in the current study, as well as calibration properties (Hanson, 2017; Helmus \& Babchishin, 2017; Singh, 2013). The former is the extent to which a risk scale can accurately discriminate between recidivists and non-recidivists (Hanson, 2017; Helmus \& Babchishin, 2017; Singh, 2013). Calibration, on the other hand, measures the degree of concordance between a scale's expected recidivism rates and observed recidivism rates in a new sample (Hanson, 2017; Helmus \& Babchishin, 2017; Singh, 2013). For instance, a scale is well calibrated if it predicts that $20 \%$ of offenders with a certain score or in a particular risk bin will reoffend and validation studies find recidivism rates close to $20 \%$ for that score or risk bin (Hanson, 2017). Future research should explore the calibration of the DRAOR for IPV recidivism to provide a more complete picture of the scale's predictive accuracy. 


\section{References}

Abbiati, M., Azzola, A., Palix, J., Gasser, J., \& Moulin, V. (2017). Validity and predictive accuracy of the structured assessment of protective factors for violence risk in criminal forensic evaluations: A Swiss cross-validation retrospective study. Criminal Justice and Behavior, 44, 493-510. doi:10.1177/0093854816677565

Abidin, Z., Davoren, M., Naughton, L., Gibbons, O., Nulty, A., \& Kennedy, H. G. (2013). Susceptibility (risk and protective) factors for in-patient violence and self-harm: Prospective study of structured professional judgement instruments START and SAPROF, DUNDRUM-3 and DUNDRUM-4 in forensic mental health services. $B M C$ Psychiatry, 13, 18. doi:10.1186/1471-244X-13-197

Agresti, A. (2007). An introduction to categorical data analysis (2nd ed.). Hoboken, NJ: WileyBlackwell.

Aldarondo, E., \& Sugarman, D. B. (1996). Risk marker analysis of the cessation and persistence of wife assault. Journal of Consulting and Clinical Psychology, 64, 1010-1019. doi:10.1037/0022-006X.64.5.1010

Almond, L., McManus, M., Brian, D., \& Merrington, D. P. (2017). Exploration of the risk factors contained within the UK's existing domestic abuse risk assessment tool (DASH): Do these risk factors have individual predictive validity regarding recidivism? Journal of Aggression, Conflict and Peace Research, 9, 58-68. doi:10.1108/JACPR-01-2016-021

American Psychiatric Association. (2013). Diagnostic and statistical manual of mental disorders (5th ed.). Arlington, VA: American Psychiatric Publishing.

American Psychological Association (2018). APA dictionary of psychology. Retrieved from https:/dictionary.apa.org/ 
Anderson, J. C., Stockman, J. K., Sabri, B., Campbell, D. W., \& Campbell, J. C. (2015). Injury outcomes in African American and African Caribbean women: The role of intimate partner violence. Journal of Emergency Nursing, 41, 36-42.

doi:10.1016/j.jen.2014.01.015

Andrews, D.A. (1980). Some experimental investigations of the principles of differential association through deliberate manipulations of the structure of service systems. American Sociological Review, 45(3), 448 478. Retrieved from https://www.jstor.org/stable/2095177?seq=1\#page_scan_tab_contents

Andrews, D. A., \& Bonta, J. (2010). The psychology of criminal conduct (5th ed.). Newark, NJ: LexisNexus/Anderson.

Andrews, D. A., \& Kiessling, J. J. (1980). Program structure and effective correctional practices: A summary of the CaVIC research. In R. Ross \& P. Gendreau (Eds.), Effective correctional treatment. Toronto, ON: Butterworth.

Averill, A. E. (2016). An investigation of the Dynamic Risk Assessment for Offender Re-entry (DRAOR) with New Zealand sexual offenders (Master's thesis). Retrieved from https://ir.canterbury.ac.nz/handle/10092/12883

Babchishin, K. M., Blais, J., \& Helmus, L. (2012). Do static risk factors predict differently for Aboriginal sex offenders? A multi-site comparison using the original and revised Static99 and Static-2002 scales. Canadian Journal of Criminology and Criminal Justice, 54, 143. doi:10.3138/cjecj.2010.E.40

Babchishin, K. M., \& Helmus, L. M. (2016). The influence of base rates on correlations: An evaluation of proposed alternative effect sizes with real-world dichotomous data. Behavior Research Methods, 48, 1021-1031. doi:10.3758/s13428-015-0627-7 
Babcock, J. C., \& Steiner, R. (1999). The relationship between treatment, incarceration, and recidivism of battering: A program evaluation of Seattle's coordinated community response to domestic violence. Journal of Family Psychology, 13, 46-59. doi:10.1037/0893-3200.13.1.46

Bakker, L., O’Malley, J. \& Riley, D. (1998). Storm warning: Statistical models for predicting violence. Christchurch, New Zealand: Psychological Service, Department of Corrections.

Baron, S. W. (2009). Differential coercion, street youth, and violent crime. Criminology: An Interdisciplinary Journal, 47, 239-268. doi:10.1111/j.1745-9125.2009.00144.x

Benda, B. B. (2005). Gender differences in life-course theory of recidivism: A survival analysis. International Journal of Offender Therapy and Comparative Criminology, 49, 325-342. doi:10.1177/0306624X04271194

Beggs, S. M., \& Grace, R. C. (2010). Assessment of dynamic risk factors: An independent validation study of the violence risk scale: Sexual offender version. Sexual Abuse: Journal of Research and Treatment, 22, 234-251. doi:10.1177/1079063210369014

Bennett, L., Goodman, L., \& Dutton, M. A. (2000). Risk assessment among batterers arrested for domestic assault: The salience of psychological abuse. Violence Against Women, 6, 1190-1203. doi:10.1177/10778010022183596

Bennett, T., Holloway, K., \& Farrington, D. (2008). The statistical association between drug misuse and crime: A meta-analysis. Aggression and Violent Behavior, 13, 107-118. doi:10.1016/j.avb.2008.02.001

Bennett Cattaneo, L., \& Goodman, L. A. (2003). Victim-reported risk factors for continued abusive behavior: Assessing the dangerousness of arrested batterers. Journal of Community Psychology, 31, 349-369. doi:10.1002/jcop.10056 
Bennett Cattaneo, L., \& Goodman, L. A. (2005). Risk factors for reabuse in intimate partner violence: A cross-disciplinary critical review. Trauma, Violence, \& Abuse, 6, 141-175. doi: $10.1177 / 1524838005275088$

Birkley, E. L., \& Eckhardt, C. I. (2015). Anger, hostility, internalizing negative emotions, and intimate partner violence perpetration: A meta-analytic review. Clinical Psychology Review, 37, 40-56. doi:10.1016/j.cpr.2015.01.002

Boccaccini, M. T., Turner, D., \& Murrie, D. C. (2008). Do some evaluators report consistently higher or lower psychopathy scores than others? Findings from a statewide sample of sexually violent predator evaluations. Psychology, Public Policy, \& Law, 14, 262-283. doi: $10.1037 / \mathrm{a} 0014523$

Bonta, J. (1996). Risk-needs assessment and treatment. In A. T. Harland (Ed.), Choosing correctional options that work: Defining the demand and evaluating the supply (pp. 1832). Thousand Oaks, CA: Sage.

Bonta, J., Blais, J., \& Wilson, H. A. (2014). A theoretically informed meta-analysis of the risk for general and violent recidivism for mentally disordered offenders. Aggression and Violent Behavior, 19, 278-287. doi:10.1016/j.avb.2014.04.014

Bonta, J., Bogue, B., Crowley, M., \& Motiuk, L. (2001). Implementing offender classification systems: Lessons learned. In G. A. Bernfeld, D. P. Farrington \& A. W. Leschied (Eds.), Offender rehabilitation in practice: Implementing and evaluating effective programs (pp. 227-245). New York, NY: John Wiley \& Sons Ltd.

Bonta, J., Bourgon, G., Rugge, T., Scott, T-L., Yessine, A. K., Gutierrez, L., \& Li, J. (2010). The Strategic Training Initiative in Community Supervision: Risk-Need-Responsivity in the real world (Research Report No. 2010-01). Ottawa, ON: Public Safety Canada. 
Bordin, E. S. (1979). The generalizability of the psychoanalytic concept of the working alliance. Psychotherapy: Theory, Research \& Practice, 16, 252-260. doi: $10.1037 / \mathrm{h} 0085885$

Borum, R. (1996). Improving the clinical practice of violence risk assessment: technology, guidelines, and training. American Psychologist, 51, 945-956. doi:10.1037/0003-066X.51.9.945

Boyle, M. H., Georgiades, K., Cullen, J., \& Racine, Y. (2009). Community influences on intimate partner violence in India: Women's education, attitudes towards mistreatment and standards of living. Social Science \& Medicine, 69, 691-697. doi:10.1016/j.socscimed.2009.06.039

Brem, M. J., Florimbio, A. R., Elmquist, J., Shorey, R. C., \& Stuart, G. L. (2018). Antisocial traits, distress tolerance, and alcohol problems as predictors of intimate partner violence in men arrested for domestic violence. Psychology of Violence, 8, 132-139. doi: $10.1037 /$ vio0000088

Brown, S. L., St. Amand, M. D., \& Zamble, E. (2009). The dynamic prediction of criminal recidivism: A three-wave prospective study. Law and Human Behavior, 33, 25-45. doi:10.1007/s10979-008-9139-7

Burczycka, M., \& Conroy, S. (2017a). Family violence in Canada: A statistical profile, 2015 (Catalogue No. 85-002-X). Retrieved from https://www150.statcan.gc.ca/n1/pub/85-002x/2017001/article/14698-eng.pdf

Burczycka, M., \& Conroy, S. (2017b). Family violence in Canada: A statistical profile, 2016 (Catalogue No. 85-002-X). Retrieved from https://www150.statcan.gc.ca/n1/pub/85-002x/2018001/article/54893-eng.pdf 
Burczycka, M., Conroy, S., \& Savage, L. (2018). Family violence in Canada: A statistical profile, 2017 (Catalogue No. 85-002-X). Retrieved from https://www150.statcan.gc.ca/n1/pub/85-002-x/2018001/article/54978-eng.pdf

Burczycka, M., \& Ibrahim, D. (2016). Family violence in Canada: A statistical profile, 2015 (Catalogue No. 85-002-X). Retrieved from https://www150.statcan.gc.ca/n1/en/pub/85002-x/2016001/article/14303-eng.pdf?st=UgoQBgVx

Butchart, A., Garcia-Moreno, C., \& Mikton, C. (2010). Preventing intimate partner and sexual violence against women: Taking action and generating evidence. Retrieved from the World Health Organization website:

http://www.who.int/violence_injury_prevention/publications/violence/9789241564007_e ng.pdf

Campbell, M. A., French, S., \& Gendreau, P. (2009). The prediction of violence in adult offenders: A meta-analytic comparison of instruments and methods of assessment. Criminal Justice and Behavior, 36, 567-590. doi:10.1177/0093854809333610

Campbell, J., Snow Jones, A., Dienneman, J., Kub, J., Schollenberger, J., O’Campo, P., Gielen, A. C., \& Wynn, C. (2002). Intimate partner violence and physical health consequences. Archives of Internal Medicine, 162(10), 1157-1163. Retrieved from https:/www.ncbi.nlm.nih.gov/pubmed/12020187

Campbell, J. C., Webster, D., Koziol-McLain, J., Block, C., Campbell, D., Curry, M. A., .. . Laughon, K. (2003). Risk factors for femicide in abusive relationships: Results from a multisite case control study. American Journal of Public Health, 93, 1089-1097. doi:10.2105/AJPH.93.7.1089 
Caetano, R., McGrath, C., Ramisetty-Mikler, S., \& Field, C. A. (2005). Drinking, alcohol problems and the five-year recurrence and incidence of male to female and female to male partner violence. Alcoholism: Clinical and Experimental Research, 29, 98-106. doi:10.1097/01.ALC.0000150015.84381.63

Cartwright, J. K., Desmarais, S. L., Hazel, J., Griffith, T., \& Azizian, A. (2018). Predictive validity of HCR-20, START, and Static-99R assessments in predicting institutional aggression among sexual offenders. Law and Human Behavior, 42, 13-25. doi:10.1037/lhb0000263

Chadwick, N. (2014). Validating the Dynamic Risk Assessment for Offender Re-entry (DRAOR) in a sample of U.S. probationers and parolees (Master's thesis). Retrieved from https://curve.carleton.ca/f43f2b17-42cf-4792-8ddc-351682dee8bf

Chaudhuri, M., \& Daly, K. (1992). Do restraining orders help? Battered women's experience with male violence and the legal process. In E. S. Buzawa \& C. G. Buzawa (Eds.), Domestic violence: The changing criminal justice response (pp. 227-252). Westport, CT: Auburn House.

Chen, H., Cohen, P., Chen, S. (2010). How big is a big odds ratio? Interpreting the magnitude of odds ratios in epidemiological studies. Communications in Statistics-Simulation and Computation, 39, 860-864. doi:10.1080/03610911003650383

Chui, W. H., \& Chan, H. C. O. (2012). Criminal recidivism among Hong Kong male juvenile probationers. Journal of Child and Family Studies, 21, 857-868. doi:10.1007/s10826-011-9546-0

Cicchetti, D. V. (2001). The precision of reliability and validity estimates re-visited: Distinguishing between clinical and statistical significance of sample size 
requirements. Journal of Clinical and Experimental Neuropsychology, 23(5), 695-700. doi:10.1076/jcen.23.5.695.1249

Clark, L. A., \& Watson, D. (1995). Constructing validity: Basic issues in objective scale development. Psychological Assessment, 7, 309-319. doi:10.1037/1040-3590.7.3.309

Coker, A. L., Smith, P. H., Bethea, L., King, M. R., \& McKeown, R. E. (2000). Physical health consequences of physical and psychological intimate partner violence. Archives of Family Medicine, 9(5), 451-457. Retrieved from https://www.ncbi.nlm.nih.gov/pubmed/10810951

Cohen, J. (1992). A power primer. Psychological Bulletin, 112, 155-159. doi:10.1037/0033-2909.112.1.155

Cox, S. M., Kochol, P., \& Hedlund, J. (2018). The exploration of risk and protective score differences across juvenile offending career types and their effects on recidivism. Youth Violence and Juvenile Justice, 16, 77-96. doi:10.1177/1541204016678439

Dahlberg, L. L., \& Krug, E. G. (2002). Violence: A global public health problem. In E. G. Krug, L. L. Dahlberg, J. A. Mercy, A. B. Zwi, \& R. Lozano (Eds.), World report on violence and health (pp. 3-21). Geneva, Switzerland: World Health Organization.

Devries, K. M., Mak, J. Y. T., García-Moreno, C., Petzold, M., Child, J. C., Falder, G., .. . Watts, C. H. (2013). The global prevalence of intimate partner violence against women. Science, 340, 1527-1528. doi:10.1126/science.1240937

de Ruiter, C., \& Nicholls, T. L. (2011). Protective factors in forensic mental health: A new frontier. International Journal of Forensic Mental Health, 10, 160-170. doi:10.1080/14999013.2011.600602 
Desmarais, S. L., Nicholls, T. L., Wilson, C. M., \& Brink, J. (2012). Using dynamic risk and protective factors to predict inpatient aggression: Reliability and validity of START assessments. Psychological Assessment, 24, 685-700. doi:10.1037/a0026668

Desmarais, S. L., \& Singh, J. P. (2013). Risk assessment instruments validated and implemented in correctional settings in the united states. Washington, DC: Council of State Governments. Retrieved from https://csgjusticecenter.org/wpcontent/uploads/2014/07/Risk-Assessment-Instruments-Validated-and-Implemented-inCorrectional-Settings-in-the-United-States.pdf

de Vogel, V., de Ruiter, C., Bouman, Y., \& de Vries Robbé, M. (2009). SAPROF: Guidelines for the assessment of protective factors for violence risk - English version. Utrecht, Netherlands: Forum Educatief.

de Vogel, V., de Ruiter, C., Bouman, Y., \& de Vries Robbé, M. (2012). SAPROF: Guidelines for the assessment of protective factors for violence risk-English version $2^{\text {nd }}$. Utrecht, Netherlands: Forum Educatief.

de Vries Robbé, M., de Vogel, V., Douglas, K. S., \& Nijman, H. L. I. (2015). Changes in dynamic risk and protective factors for violence during inpatient forensic psychiatric treatment: Predicting reductions in postdischarge community recidivism. Law and Human Behavior, 39, 53-61. doi:10.1037/lhb0000089

Douglas, K. S., Hart, S. D., Webster, C. D., \& Belfrage, H. (2013). HCR-20 ${ }^{V 3}$ : Assessing risk of violence - User guide. Burnaby, Canada: Mental Health, Law, and Policy Institute, Simon Fraser University. 
Douglas, K. S., \& Skeem, J. L. (2005). Violence risk assessment: Getting specific about being dynamic. Psychology, Public Policy, and Law, 11, 347-383. doi:10.1037/1076-8971.11.3.347

Dutton, D. G. (1985). An ecologically nested theory of male violence toward intimates. International Journal of Women's Studies, 8(4), 404-413. Retrieved from http://psycnet.apa.org/psycinfo/1987-07556-001

Dutton, M. A., Green, B. L., Kaltman, S. I., Roesch, D. M., Zeffiro, T. A., \& Krause, E. D. (2006). Intimate partner violence, PTSD, and adverse health outcomes. Journal of Interpersonal Violence, 21, 955-968. doi:10.1177/0886260506289178

Dowden, C., \& Andrews, D. A. (2004). The importance of staff practice in delivering effective correctional treatment: A meta-analytic review of core correctional practice. International Journal of Offender Therapy and Comparative Criminology, 48, 203-214. doi:10.1177/0306624X03257765

Eckhardt, C. I. (2007). Effects of alcohol intoxication on anger experience and expression among partner assaultive men. Journal of Consulting and Clinical Psychology, 75, 61-71. doi:10.1037/0022-006X.75.1.61

Edens, J. F., \& Boccaccini, M. T. (2017). Taking forensic mental health assessment "out of the lab" and into "the real world": Introduction to the special issue on the field utility of forensic assessment instruments and procedures. Psychological Assessment, 29, 599-610. doi: $10.1037 /$ pas0000475

Edwards, K. M. (2015). Intimate partner violence and the rural-urban-suburban divide: Myth or reality? A critical review of the literature. Trauma, Violence, \& Abuse, 16, 359-373. doi:10.1177/1524838014557289 
Fals-Stewart, W. (2003). The occurrence of partner physical aggression on days of alcohol consumption: A longitudinal diary study. Journal of Consulting and Clinical Psychology, 71, 41-52. doi:10.1037/0022-006X.71.1.41

Fanslow, J., \& Gulliver, P. (2015). Exploring risk and protective factors for recent and past intimate partner violence against New Zealand women. Violence and Victims, 30, 960-983. doi:10.1891/0886-6708.VV-D-14-00010

Feder, L., \& Dugan, L. (2002). A test of the efficacy of court-mandated counseling for domestic violence offenders: The Broward experiment. Justice Quarterly, 19, 343- 375. doi:10.1080/07418820200095271

Ferguson, A. M. (2016). An age-old issue: Evaluating the applicability of adult criminal assessment tools for use with youth offenders (Master's thesis). Retrieved from http://researcharchive.vuw.ac.nz/xmlui/bitstream/handle/10063/4861/thesis.pdf?sequence $=1$

Field, A. (2009). Discovering statistics using SPSS ( $3^{\text {rd }}$ ed.). Thousand Oaks, CA: Sage.

Fiqia, N. A., Lang, R. A., Plutchik, R., \& Holden, R. (1987). Personality differences between sex and violent offenders. International Journal of Offender Therapy and Comparative Criminology, 31, 211-226. doi:10.1177/0306624X8703100304

Flake, J. K., Pek, J., \& Hehman, E. (2017). Construct validation in social and personality research: Current practice and recommendations. Social Psychological and Personality Science, 8, 370-378. doi:10.1177/1948550617693063

Flores, A. W., Lowenkamp, C. T., Holsinger, A. M., \& Latessa, E. J. (2006). Predicting outcome with the level of service inventory-revised: The importance of implementation integrity. Journal of Criminal Justice, 34, 523-529. doi:10.1016/j.jcrimjus.2006.09.007 
Ford, D. A., \& Regoli, M. J. (1992). The preventive impacts of policies for prosecuting wife batterers. In E. S. Buzawa \& C. G. Buzawa (Eds.), Domestic violence: The changing criminal justice response (pp. 181-207). Westport, CT: Auburn House.

Gehlbach, H., \& Brinkworth, M. E. (2011). Measure twice, cut down error: A process for enhancing the validity of survey scales. Review of General Psychology, 15, 380-387. doi:10.1037/a0025704

Gerberding, J. L., Binder, S., Hammond, W. R., Arias, I. (2003). Cost of intimate partner violence against women in the United States. Retrieved from Centers for Disease Control and Prevention website: https://www.cdc.gov/violenceprevention/pdf/IPVBook-a.pdf

Gondolf, E. W. (1997). Patterns of reassault in batterer programs. Violence and Victims, 12(4), 373-387. Retrieved from https://www.ncbi.nlm.nih.gov/pubmed/9591355

Gordon, J. A., \& Moriarty, L. J. (2003). The effects of domestic violence batterer treatment on domestic violence recidivism: The Chesterfield County experience. Criminal Justice and Behavior, 30, 118-134. doi:10.1177/0093854802239166

Gout, N. D. (2011). Cultural identity and familial relationships as protective factors against intimate partner violence among American Indian and Alaska Native mothers (Doctoral dissertation) Retrieved from PsycINFO (Order No. AAI3411732).

Gray, A. L. (2016). Assessing risk for intimate partner violence: A cross-validation of the ODARA and DVRAG within a sample of incarcerated offenders (Master's thesis). Retrieved from https://curve.carleton.ca/72e3aeb0-7550-4f38-9f47-d50f33431895

Greiner, L. E., Law, M. A., \& Brown, S. L. (2015). Using dynamic factors to predict recidivism among women: A four-wave prospective study. Criminal Justice and Behavior, 42, 457480. doi: $10.1177 / 0093854814553222$ 
Guy, L. S. (2010). Performance indicators of the structured professional judgment approach for assessing risk for violence to others: A meta-analytic survey (Doctoral dissertation). Retrieved from ProQuest Dissertations \& Theses Global. (Order No. NR58733).

Haines, A., Khan, F., Noblett, S., Omodunbi, O., Sadiq, K., Zaman, W., \& Whittington, R. (2018). Assessing protective factors for violence risk in U.K. general mental health services using the Structured Assessment of Protective Factors. International Journal of Offender Therapy and Comparative Criminology. Advance online publication. doi:10.1177/0306624X17749449

Hamberger, L. K., \& Hastings, J. E. (1990). Recidivism following spouse abuse abatement counseling: Treatment program implications. Violence and Victims, 5(3), 157-170. Retrieved from https://www.ncbi.nlm.nih.gov/pubmed/2275895

Hanby, L. (2013). A longitudinal study of dynamic risk, protective factors, and criminal recidivism: Change over time and the impact of assessment timing (Doctoral dissertation). Retrieved from https://curve.carleton.ca/b6aa7d5d-f44b-48d6-9ea9$5 \mathrm{~d} 0 \mathrm{f} 92 \mathrm{~b} 8 \mathrm{f} 7 \mathrm{a} 7$

Hanley, J. A., \& McNeil, B. J. (1982). The meaning and use of the area under a receiver operating characteristic (ROC) curve. Radiology, 143, 29-36. doi:10.1148/radiology.143.1.7063747

Hanson, R. K. (2017). Assessing the calibration of actuarial risk scales: A primer on the E/O index. Criminal Justice and Behavior, 44, 26-39. doi:10.1177/0093854816683956

Hanson, R. K., \& Harris, A. J. R. (1998). Dynamic predictors of sexual recidivism (Report No. 1998-1). Ottawa, ON: Public Safety Canada. 
Hanson, R. K., \& Harris, A. J. R. (2000). Where should we intervene?: Dynamic predictors of sexual offense recidivism. Criminal Justice and Behavior, 27, 6-35. doi:10.1177/0093854800027001002

Hanson, R. K., Harris, A. J. R., Scott, T., \& Helmus, L. (2007). Assessing the risk of sexual offenders on community supervision: The dynamic supervision project (Report No. 200705). Ottawa, ON: Public Safety Canada.

Hanson, R. K., \& Morton-Bourgon, K. (2009). The accuracy of recidivism risk assessments for sexual offenders: A meta-analysis of 118 prediction studies. Psychological Assessment, 21, 1-21. doi:10.1037/a0014421

Harrell, A., \& Smith, B. A. (1996). Effects of restraining orders on domestic violence victims. In E. S. Buzawa \& C. G. Buzawa (Eds.), Do arrests and restraining orders work? (pp. 214242). Thousand Oaks, CA: Sage.

Hart, S. D., \& Cooke, D. J. (2013). Another look at the (im-)precision of individual risk assessments made using actuarial risk assessment instruments. Behavioral Sciences and the Law, 31, 81-102. doi:10.1002/bs1.2049

Hawkins, J. D., Catalano, R. F., \& Miller, J. Y. (1992). Risk and protective factors for alcohol and other drug problems in adolescence and early adulthood: Implications for substance abuse prevention. Psychological Bulletin, 112, 64-105. doi:10.1037/0033-2909.112.1.64

Helmus, L. M., \& Babchishin, K. M. (2017). Primer on risk assessment and the statistics used to evaluate its accuracy. Criminal Justice and Behavior, 44, 8-25. doi:10.1177/0093854816678898

Helmus, L., Babchishin, K. M., \& Blais, J. (2012). Predictive accuracy of dynamic risk factors for Aboriginal and non-Aboriginal sex offenders: A comparison using STABLE-2007. 
International Journal of Offender Therapy and Comparative Criminology, 56, 856-876. doi:10.1177/0306624X11414693

Helmus, L., \& Bourgon, G. (2011). Taking stock of 15 years of research on the Spousal Assault Risk Assessment Guide (SARA): A critical review. International Journal of Forensic Mental Health, 10, 64-75. doi:10.1080/14999013.2010.551709

Helmus, L., Thornton, D., Hanson, R. K., \& Babchishin, K. M. (2012). Improving the predictive accuracy of Static-99 and Static-2002 with older sex offenders revised age weights. Sexual Abuse: A Journal of Research and Treatment, 24, 64-101. doi:10.1177/1079063211409951

Hilton, N. Z. (1992). Battered women's concerns about their children witnessing wife assault. Journal of Interpersonal Violence, 7, 77-86. doi:10.1177/088626092007001007

Hilton, N. Z., \& Harris, G. T. (2005). Predicting wife assault: A critical review and implications for policy and practice. Trauma, Violence, \& Abuse, 6, 3-23. doi: $10.1177 / 1524838004272463$

Hilton, N. Z., Harris, G. T., \& Rice, M. E. (2006). Sixty-six years of research on the clinical versus actuarial prediction of violence. The Counseling Psychologist, 34, 400-409. doi: $10.1177 / 0011000005285877$

Hilton, N. Z., Harris, G. T., \& Rice, M. E. (2010). Risk assessment for domestically violent men: Tools for criminal justice, offender intervention, and victim services. Washington, DC: American Psychological Association.

Hilton, N. Z., Harris, G. T., Rice, M. E., Lang, C., Cormier, C. A., \& Lines, K. (2004). A brief actuarial assessment for the prediction of wife assault recidivism: The Ontario Domestic 
Assault Risk Assessment. Psychological Assessment, 16, 267-275.

doi:10.1037/1040-3590.16.3.267

Hogan, N. R., \& Olver, M. E. (2016). Assessing risk for aggression in forensic psychiatric inpatients: An examination of five measures. Law and Human Behavior, 40, 233-243. doi: $10.1037 / \mathrm{lhb} 0000179$

Howells, K. (2004). Anger and its links to violent offending. Psychiatry, Psychology and Law, 11, 189-196. doi:10.1375/1321871042707278

Hudson, S. M., Ward, T., \& McCormack, J. C. (1999). Offense pathways in sexual offenders. Journal of Interpersonal Violence, 14, 779-798. doi:10.1177/088626099014008001

Iowa Department of Corrections. (2003). Iowa classification system - assessment and reassessment of risk: Instructions scoring guide. Unpublished user-manual.

Jacobson, N. S., Gottman, J. M., Gortner, E., Berns, S., \& Shortt, J. W. (1996). Psychological factors in the longitudinal course of battering: When do the couples split up? when does the abuse decrease? Violence and Victims, 11(4), 371-392. Retrieved from https://www.ncbi.nlm.nih.gov/pubmed/9210278

Jaffe, P. G., Wolfe, D. A., \& Wilson, S. K. (1990). Children of battered women. Newbury Park, CA: Sage.

Jung, S., \& Buro, K. (2017). Appraising risk for intimate partner violence in a police context. Criminal Justice and Behavior, 44, 240-260. doi:10.1177/0093854816667974

Kahui, S., \& Snively, S. (2014). Measuring the economic costs of child abuse and intimate partner violence to New Zealand. Retrieved from New Zealand Family Violence Clearinghouse website: 
http://ndhadeliver.natlib.govt.nz/delivery/DeliveryManagerServlet?dps_pid=IE25595234 \&dps_custom_att_1 $=\mathrm{ilsdb}$

Kennealy, P. J., Skeem, J. L., Eno Louden, J., \& Manchak, S. M. (2012). Firm, fair, and caring officer-offender relationships protect against supervision failure. Law and Human Behavior, 36, 496-505. doi:10.1037/h0093935

Kouyoumdjian, F. G., Calzavara, L. M., Bondy, S. J., O'Campo, P., Serwadda, D., Nalugoda, F., .. . Gray, R. (2013). Intimate partner violence is associated with incident HIV infection in women in Uganda. Aids, 27, 1331-1338. doi:10.1097/QAD.0b013e32835fd851

KPMG. (2016). The cost of violence against women and their children in Australia - Final Report. Retrieved from Australian Government Department of Social Services website: https://www.dss.gov.au/sites/default/files/documents/08_2016/the_cost_of_violence_agai nst_women_and_their_children_in_australia_-_final_report_may_2016.pdf

Kropp, P. R., \& Hart, S. D. (2000). The Spousal Assault Risk Assessment (SARA) guide: Reliability and validity in adult male offenders. Law and Human Behavior, 24, 101-118. doi:10.1023/A:1005430904495

Kropp, P. R., Hart, S. D., Webster, C. W., \& Eaves, D. (1994). Manual for the Spousal Assault Risk Assessment Guide. Vancouver, BC: British Columbia Institute on Family Violence.

Kropp, P. R., Hart, S. D., Webster, C. W., \& Eaves, D. (1995). Manual for the Spousal Assault Risk Assessment Guide, 2nd ed. Vancouver, BC: British Columbia Institute on Family Violence.

Kropp, P. R., Hart, S. D., Webster, C. W., \& Eaves, D. (1998). Spousal Assault Risk Assessment: User's Guide. Toronto: Multi-Health Systems, Inc. 
Kutner, M., Nachtsheim, C., Neter, J., \& Li, W. (2005). Applied linear statistical models $\left(5^{\text {th }}\right.$ ed.). New York, NY: McGraw-Hill Irwin.

LaMotte, A. D., Meis, L. A., Winters, J. J., Barry, R. A., \& Murphy, C. M. (2018). Relationship problems among men in treatment for engaging in intimate partner violence. Journal of Family Violence, 33, 75-82. doi:10.1007/s10896-017-9920-9

Latessa, E. J., Lemke, R., Makarios, M., Smith, P., \& Lowenkamp, C. T. (2010). The creation and validation of the Ohio Risk Assessment System (ORAS). Federal Probation, 74(1), 16-22. Retrieved from https://www.uc.edu/content/dam/uc/ics/docs/LatessaEtAlTheCreationAndValidation.pdf

Latessa, E. J., \& Lovins, B. (2010). The role of offender risk assessment: A policy maker guide. Victims and Offenders, 5, 203-219. doi:10.1080/15564886.2010.485900

Laub, J. H., Nagin, D. S., \& Sampson, R. J. (1998). Trajectories of change in criminal offending: Good marriages and the desistance process. American Sociological Review, 63, 225-238. doi: $10.2307 / 2657324$

Laub, J. H., \& Sampson, R. J. (2001). Understanding desistance from crime. Crime and Justice, 28(1), 1-69. Retrieved from https://dash.harvard.edu/bitstream/handle/1/3226958/Sampson_UnderstandingDesistance .pdf? sequence $=4$

Leonard, K. E., \& Senchak, M. (1996). Prospective prediction of husband marital aggression within newlywed couples. Journal of Abnormal Psychology, 105, 369-380. doi:10.1037/0021-843X.105.3.369 
Leone, R. M., Crane, C. A., Parrott, D. J., \& Eckhardt, C. I. (2016). Problematic drinking, impulsivity, and physical IPV perpetration: A dyadic analysis. Psychology of Addictive Behaviors, 30, 356-366. doi:10.1037/adb0000159

Lewis, K., Olver, M. E., \& Wong, S. C. (2012). The Violence Risk Scale: Predictive validity and linking changes in risk with violent recidivism in a sample of high-risk offenders with psychopathic traits. Assessment, 20, 150-64. doi:10.1177/1073191112441242

Lloyd, C. (2015). Can a dynamic risk instrument make short-term predictions in "real time”? Developing a framework for testing proximal assessment of offender recidivism risk during re-entry (Unpublished doctoral dissertation). Carleton University, Ottawa, ON.

Lodewijks, H. P. B., de Ruiter, C., \& Doreleijers, T. A. H. (2010). The impact of protective factors in desistance from violent reoffending: A study in three samples of adolescent offenders. Journal of Interpersonal Violence, 25, 568-587.

doi:10.1177/0886260509334403

Loevinger, J. (1957). Objective tests as instruments of psychological theory. Psychological Reports, 3, 635-694. doi:10.2466/PR0.3.7.635-694

Lowder, E. M., Desmarais, S. L., Rade, C. B., Coffey, T., \& Van Dorn, R. A. (2017). Models of protection against recidivism in justice-involved adults with mental illnesses. Criminal Justice and Behavior, 44, 893-911. doi:10.1177/0093854817710966

Lowder, E. M., Desmarais, S. L., Rade, C. B., Johnson, K. L., \& Van Dorn, V. A. (2017). Reliability and validity of START and LSI-R assessments in mental health jail diversion clients. Assessment. Advance online publication. doi:10.1177/1073191117704505 
Lowenkamp, C. T., Johnson, J. L., Trevino, P., \& Serin, R. C. (2016). Enhancing community supervision through the application of dynamic risk assessment. Federal Probation, 80(2), 16-20. Retrieved from http://www.uscourts.gov/sites/default/files/80_2_3_0.pdf

Mann, H. B., \& Whitney, D. R. (1947). On a test of whether one of two random variables is stochastically larger than the other. Annals of Mathematical Statistics, 18, 50-60. doi:10.1214/aoms/1177730491

Martin, D. J., Garske, J. P., \& Davis, M. K. (2000). Relation of the therapeutic alliance with outcome and other variables: A meta-analytic review. Journal of Consulting and Clinical Psychology, 68, 438-450. doi:10.1037/0022-006X.68.3.438

Maruna, S. (2001). Making good: How ex-convicts reform and rebuild their lives. American Psychological Association, Washington, DC. doi:10.1037/10430-000

Maume, M. O., Ousey, G. C., \& Beaver, K. (2005). Cutting the grass: A reexamination of the link between marital attachment, delinquent peers and desistance from marijuana use. Journal of Quantitative Criminology, 21, 27-53. doi:10.1007/s10940-004-1786-3

Max, W., Rice, D. P., Finkelstein, E., Bardwell, R. A., \& Leadbetter, S. (2004). The economic toll of intimate partner violence against women in the United States. Violence and Victims, 19, 259-272. doi:10.1891/vivi.19.3.259.65767

McCarroll, J. E., Thayer, L. E., Liu, X., Newby, J. H., Norwood, A. E., Fullerton, C. S., \& Ursano, R. J. (2000). Spouse abuse recidivism in the U.S. army by gender and military status. Journal of Consulting and Clinical Psychology, 68, 521-525. doi:10.1037/0022-006X.68.3.521 
McCoy, L. A., \& Miller, H. A. (2013). Comparing gender across risk and recidivism in nonviolent offenders. Women \& Criminal Justice, 23, 143-162. doi:10.1080/08974454.2012.759054

McFadgion, A. (2014). Intimate partner violence and probable traumatic brain injury: Manifestations in the lives of abused women (Doctoral dissertation). Retrieved from ProQuest Dissertations \& Theses Global. (UMI No. 3613786).

Meloy, J. R., Cowett, P. Y., Parker, S. B., Hofland, B., \& Friedland, A. (1997). Domestic protection orders and the prediction of subsequent criminality and violence toward protectees. Psychotherapy: Theory, Research, Practice, Training, 34, 447-458. doi: $10.1037 / \mathrm{h} 0087850$

Messing, J. T., \& Thaller, J. (2013). The average predictive validity of intimate partner violence risk assessment instruments. Journal of Interpersonal Violence, 28, 1537-1558. doi:10.1177/0886260512468250

Miller, J. L., \& Krull, A. C. (1997). Controlling domestic violence: Victim resources and police intervention. In G. Kaufman Kantor \& J. L. Jasinski (Eds.), Out of the dark- ness: Contemporary perspectives on family violence (pp. 235-254). Thousand Oaks, CA: Sage.

Miller, H. A. (2006a). Inventory of Offender Risk, Needs, and Strengths: A professional manual. Lutz, FL: Psychological Assessment Resources

Miller, H. A. (2006b). A dynamic assessment of offender risk, needs, and strengths in a sample of general offenders. Behavioural Sciences and the Law, 24, 767-782. doi:10.1002/bsl.728 
Miller, H. A. (2015). Protective strengths, risk, and recidivism in a sample of known sexual offenders. Sexual Abuse: Journal of Research and Treatment, 27, 34-50. doi:10.1177/1079063214564389

Moffitt, T. E. (1993). Adolescence-limited and life-course-persistent antisocial behavior: A developmental taxonomy. Psychological Review, 100, 674-701. doi:10.1037/0033-295X.100.4.674

Morgan, R. D., Kroner, D. G., Mills, J. F., Serna, C., \& McDonald, B. (2013). Dynamic risk assessment: A validation study. Journal of Criminal Justice, 41, 115-124. doi:10.1016/j.jcrimjus.2012.11.004

Muirhead, J. (2015). Risky business: Evaluating the Dynamic Risk Assessment for Offender Reentry for use with New Zealand youth (Master's thesis). Retrieved from http://researcharchive.vuw.ac.nz/xmlui/bitstream/handle/10063/5221/thesis.pdf?sequence $=1$

Murphy, C. M., Morrel, T. M., Elliott, J. D., \& Neavins, T. M. (2003). A prognostic indicator scale for the treatment of partner abuse perpetrators. Journal of Interpersonal Violence, 18, 1087-1105. doi:10.1177/0886260503254515

Murrie, D. C., Boccaccini, M., Johnson, J., \& Janke, C. (2008). Does interrater (dis)agreement on Psychopathy Checklist scores in Sexually Violent Predator trials suggest partisan allegiance in forensic evaluation? Law and Human Behavior, 32, 352-362. doi:10.1007/s10979-007-9097-5

Norlander, B., \& Eckhardt, C. (2005). Anger, hostility, and male perpetrators of intimate partner violence: A meta-analytic review. Clinical Psychology Review, 25, 119-152. doi:10.1016/j.cpr.2004.10.001 
Nyamathi, A. M., Salem, B. E., Hall, E., Oleskowicz, T., Ekstrand, M., Yadav, K., .. . Faucette, M. (2017). Violent crime in the lives of homeless female ex-offenders. Issues in Mental Health Nursing, 38, 122-131. doi:10.1080/01612840.2016.1253807

Olver, M. E., \& Jung, S. (2017). Incremental prediction of intimate partner violence: An examination of three risk measures. Law and Human Behavior, 41, 440-453. doi: $10.1037 / \mathrm{lhb} 0000251$

Olver, M. E., Wong, S. C. P., Nicholaichuk, T., \& Gordon, A. (2007). The validity and reliability of the Violence Risk Scale-Sexual Offender Version: Assessing sex offender risk and evaluating therapeutic chance. Psychological Assessment, 19, 318-329. doi:10.1037/1040-3590.19.3.318

Palmer, S. E., Brown, R. A., \& Barrera, M. E. (1992). Group treatment program for abusive husbands: Long-term evaluation. American Journal of Orthopsychiatry, 62, 276-283. doi: $10.1037 / \mathrm{h} 0079336$

Pan, Y., \& Jackson, R. T. (2008). Ethnic difference in the relationship between acute inflammation and serum ferritin in US adult males. Epidemiology \& Infection, 136, 421431. doi:10.1017/S095026880700831X

Pardoel, K. (2018). An examination of the influence of race and gender on dynamic risk assessment (Doctoral dissertation in preparation). Carleton University, Ottawa, ON.

Pattee, D., \& Farber, B. A. (2008). Patients' experiences of self-disclosure in psychotherapy: The effects of gender and gender role identification. Psychotherapy Research, 18(3), 306-315. doi:10.1080/10503300701874534 
Penney, S. R., Marshall, L. A., \& Simpson, A. I. F. (2016). The assessment of dynamic risk among forensic psychiatric patients transitioning to the community. Law and Human Behavior, 40, 374-386. doi:10.1037/lhb0000183

Perley-Robertson, B., Helmus, L. M., \& Forth, A. (2018). Predictive accuracy of static risk factors for Canadian Indigenous offenders compared to non-Indigenous offenders: Implications for risk assessment scales. Psychology, Crime \& Law. Advance online publication. doi:10.1080/1068316X.2018.1519827

Persson, M., Belfrage, H., Fredriksson, B., \& Kristiansson, M. (2017). Violence during imprisonment, forensic psychiatric care, and probation: Correlations and predictive validity of the risk assessment instruments COVR, LSI-R, HCR-20 ${ }^{\mathrm{V} 3}$, and SAPROF. The International Journal of Forensic Mental Health, 16, 117-129. doi:10.1080/14999013.2016.1266420

Phenix, A., Fernandez, Y., Harris, A. J. R., Helmus, M., Hanson, R. K., \& Thornton, D. (2016). Static-99R coding rules revised - 2016. Retrieved from http://www.static99.org/pdfdocs/Coding_manual_2016_v2.pdf

Philipse, M. W. G., Koeter, M. W. J., van der Staak, C. P. F., \& van den Brink, W. (2006). Static and dynamic patient characteristics as predictors of criminal recidivism: A prospective study in a Dutch forensic psychiatric sample. Law and Human Behavior, 30, 309-327. doi:10.1007/s10979-006-9013-4

Polaschek, D. L. L. (2016). Desistance and dynamic risk factors belong together. Psychology, Crime \& Law, 22, 171-189. doi:10.1080/1068316X.2015.1114114 
Pratt, T. C., \& Cullen, F. T. (2000). The empirical status of Gottfredson and Hirschi's general theory of crime: A meta-analysis. Criminology, 38, 931-964. doi:10.1111/j.1745-9125.2000.tb00911.x

Prell, L., Vitacco, M. J., \& Zavodny, D. (2016). Predicting violence and recidivism in a large sample of males on probation or parole. International Journal of Law and Psychiatry, 49, 107-113. doi:10.1016/j.ijlp.2016.08.003

Prison Legal News. (2013, May). Iowa sex offender special sentence provisions interpreted: Time-served credit, but not earned-time, reduces revocation term. Prison Legal News, 24(5), 25. Retrieved from https://www.prisonlegalnews.org/news/2013/may/15/iowa-sexoffender-special-sentence-provisions-interpreted-time-served-credit-but-not-earned-timereduces-revocation-term/

Reynolds, C. R., \& Livingstone, R. B. (2012). Mastering modern psychological testing: Theory and methods. Harlow, UK: Pearson Education Limited.

Rice, M. E., \& Harris, G. T. (2005). Comparing effect sizes in follow-up studies: ROC area, Cohen's $d$, and r. Law and Human Behavior, 29, 615-620. doi:10.1007/s10979-005-6832-7

Richard, K. M. (2013). Predicting persistence and desistence of recidivism in youth offenders: The role of risk and protective factors in criminal offending (Doctoral dissertation). Retrieved from PsycINFO. (Order No. AAINR83221)

Rutter, M. (1985). Resilience in the face of adversity: Protective factors and resistance to psychiatric disorder. The British Journal of Psychiatry, 147, 598-611. doi:10.1192/bjp.147.6.598 
Salerno, J., Marshall, V. D., \& Picken, E. B. (2012). Validity and reliability of the rapid assessment for adolescent preventive services adolescent health risk assessment. Journal of Adolescent Health, 50, 595-599. doi:10.1016/j.jadohealth.2011.10.015

Sampson, R. J., \& Laub, J. H. (2005). A life-course view of the development of crime. Annals of the American Academy of Political and Social Science, 602, 12-45. doi: $10.1177 / 0002716205280075$

Scott-Storey, K., Wuest, J., \& Ford-Gilboe, M. (2009). Intimate partner violence and cardiovascular risk: Is there a link? Journal of Advanced Nursing, 65, 2186-2197. doi:10.1111/j.1365-2648.2009.05086.x

Sellers, C. S., Cochran, J. K., \& Branch, K. A. (2005). Social learning theory and partner violence: A research note. Deviant Behavior, 26, 379-395. doi:10.1080/016396290931669

Serin, R. C. (2007, 2015, 2017). The Dynamic Risk Assessment Scale for Offender Re-entry (DRAOR): Guidelines for case planning and risk management using structured assessment of dynamic risk and protective factors. Unpublished user-manual.

Serin, R. C. (2016). Dynamic Risk Assessment for Offender Re-entry: Training curriculum. Unpublished lecture.

Serin, R. C., \& Chadwick, N. (2017). Proposed DRAOR decision rules and case plan strategies. Unpublished user-manual.

Serin, R. C., Chadwick, N., \& Prell, L. (2018). Dynamic risk and protective factors. Unpublished manuscript. 
Serin, R. C., Gobeil, R., Lloyd, C. D., Chadwick, N., Wardrop, K., \& Hanby, L. (2016). Using dynamic risk to enhance conditional release decisions in prisoners to improve their outcomes. Behavioral Sciences \& the Law, 34, 321-336. doi:10.1002/bs1.2213

Serin, R. C., \& Lloyd, C. D. (2009). Examining the process of offender change: The transition to crime desistance. Psychology, Crime \& Law, 15, 347-364.

doi:10.1080/10683160802261078

Serin, R. C., Lloyd, C., \& Hanby, L. J. (2010). Enhancing offender re-entry: An integrated model for understanding offender re-entry. European Journal of Probation, 2(2), 53-75. doi:10.1177/206622031000200205

Serin, R. C., \& Lowenkamp, C. T. (2015). Selecting and using risk and need assessments. National Drug Court Institute: Drug Court Practitioner Fact Sheet. Retrieved from https://www.ndci.org/wp-content/uploads/Fact\%20Sheet\%20Risk\%20Assessment.pdf

Serin, R. C., Lowenkamp, C. T., \& Lloyd, C. D. (in press). Managing violent offenders in the community: Reentry and beyond. In J. S. Wormith, L. A. Craig, \& T. Hogue (Eds.) What works in violence risk management: Theory, research and practice. Hoboken, New Jersey: Wiley-Blackwell.

Serin, R. C., Mailloux, D. L., \& Wilson, N. J. (2012) The Dynamic Risk Assessment for Offender Re-entry (DRAOR). Unpublished user manual.

Serin, R. C., \& Wilson, N. J. (2013, 2017). Structured Dynamic Assessment Case-management 21 item (SDAC-21): Guidelines for case management using structured assessment of dynamic risk, responsivity, and protective factors. Unpublished user-manual.

Shepard, M. F. (1992). Predicting batterer recidivism five years after community intervention. Journal of Family Violence, 7, 167-178. doi:10.1007/BF00979025 
Sher, M. A., Warner, L., McLean, A., Rowe, K., \& Gralton, E. (2017). A prospective validation study of the START:AV. Journal of Forensic Practice, 19, 115-129. doi:10.1108/JFP-10-2015-0049

Shorey, R. C., Brasfield, H., Febres, J., \& Stuart, G. L. (2011). The association between impulsivity, trait anger, and the perpetration of intimate partner and general violence among women arrested for domestic violence. Journal of Interpersonal Violence, 26, 2681-2697. doi:10.1177/0886260510388289

Shorey, R. C., McNulty, J. K., Moore, T. M., \& Stuart, G. L. (2015). Emotion regulation moderates the association between proximal negative affect and intimate partner violence perpetration. Prevention Science, 16, 873-880. doi:10.1007/s11121-015-0568-5

Silverman, J. G., Decker, M. R., Saggurti, N., Balaiah, D., \& Raj, A. (2008). Intimate partner violence and HIV infection among married Indian women. JAMA: Journal of the American Medical Association, 300, 703-710. doi:10.1001/jama.300.6.

Singh, J. P. (2013). Predictive validity performance indicators in violence risk assessment: A methodological primer. Behavioral Sciences \& the Law, 31, 8-22. doi:10.1002/bsl.2052

Skeem, J. L., Louden, J. E., Polaschek, D., \& Camp, J. (2007). Assessing relationship quality in mandated community treatment: Blending care with control. Psychological Assessment, 19, 397-410. doi:10.1037/1040-3590.19.4.397

Skeem, J., \& Mulvey, E. (2002). Monitoring the violence potential of mentally disordered offenders being treated in the community. In A. Buchanan (Ed.), Care of the mentally disordered offender in the community (pp. 111-142). New York, NY: Oxford Press. 
Skelton, A., Riley, D., Wales, D., \& Vess, J. (2006). Assessing risk for sexual offenders in New Zealand: Development and validation of a computer-scored risk measure. Journal of Sexual Aggression, 12, 277-286. doi:10.1080/13552600601100326

Smeth, A. (2013). Evaluating risk assessments among sex offenders: a comparative analysis of static and dynamic factors (Master's thesis). Retrieved from https://curve.carleton.ca/209c6f9c-c244-438a-ad60-80c72225e93a

Smeth, A. (2018). [SDAC-21 inter-rater reliability]. Unpublished raw data.

Smith, S. G., Chen, J., Basile, K. C., Gilbert, L. K., Merrick, M. T., Patel, N., Walling, M., \& Jain, A. (2017). The National Intimate Partner and Sexual Violence Survey (NISVS): 2010-2012 state report. Retrieved from Centers for Disease Control and Prevention website: https://www.cdc.gov/violenceprevention/pdf/NISVS-StateReportBook.pdf

Snow Jones, A., \& Gondolf, E. W. (2001). Time-varying risk factors for reassault among batterer program participants. Journal of Family Violence, 16, 345-359.

doi:10.1023/A:1012268725273

Stöck1, H., Devries, K., Rotstein, A., Abrahams, N., Campbell, J., Watts, C., \& Moreno, C. G. (2013). The global prevalence of intimate partner homicide: A systematic review. The Lancet, 382, 859-865. doi:10.1016/S0140-6736(13)61030-2

Stouthamer-Loeber, M., Wei, E., Loeber, R., \& Masten, A. S. (2004). Desistance from persistent serious delinquency in the transition to adulthood. Development and Psychopathology, 16, 897-918. doi:10.1017/S0954579404040064

Swets, J. A., Dawes, R. M., \& Monahan, J. (2000). Psychological science can improve diagnostic decisions. Psychological Science in the Public Interest, 1, 1-26. doi:10.1111/1529-1006.001 
Tabachnick, B. G., \& Fidell, L. S. (2013). Using multivariate statistics: Pearson new international edition (6th ed.). London, England: Pearson.

Tamatea, A., \& Wilson, N. (2009). Dynamic Risk Assessment for Offender Re-entry (DRAOR): A pilot study (Unpublished report). Christchurch, New Zealand: New Zealand Department of Corrections.

Taylor, B. G., Davis, R. C., \& Maxwell, C. D. (2001). The effects of a group batterer treatment program: A randomized experiment in Brooklyn. Justice Quarterly, 18, 171-201. doi:10.1080/07418820100094861

Tollefson, D. R., \& Gross, E. R. (2006). Predicting recidivism following participation in a treatment program for batterers. Journal of Social Service Research, 32, 39-62. doi:10.1300/J079v32n04_03

Tripodi, S. J., Kim, J. S., \& Bender, K. (2010). Is employment associated with reduced recidivism?: The complex relationship between employment and crime. International Journal of Offender Therapy and Comparative Criminology, 54, 706-720. doi:10.1177/0306624X09342980

Turner, A. P. (2001). Exploring the role of negative mood states in the substance use and delinquency of incarcerated adolescents (Doctoral dissertation). Available from ProQuest Dissertations and Theses database. (Order No. AAI3014043).

Turner, D., Rettenberger, M., Yoon, D., Klein, V., Eher, R., \& Briken, P. (2016). Risk assessment in child sexual abusers working with children. Sexual Abuse: Journal of Research and Treatment, 28, 572-596. doi:10.1177/1079063214564390

Uggen, C. (1999). Ex-offenders and the conformist alternative: A job quality model of work and crime. Social Problems, 46, 127-151. doi:10.1525/sp.1999.46.1.03x0245k 
Uggen, C. (2000). Work as a turning point in the life course of criminals: A duration model of age, employment, and recidivism. American Sociological Review, 65, 529-546. doi: $10.2307 / 2657381$

Ullrich, S., \& Coid, J. (2011). Protective factors for violence among released prisoners: Effects over time and interactions with static risk. Journal of Consulting and Clinical Psychology, 79, 381-390. doi:10.1037/a0023613

Valera, E., \& Kucyi, A. (2017). Brain injury in women experiencing intimate partner-violence: Neural mechanistic evidence of an "invisible" trauma. Brain Imaging and Behavior, 11(6), 1664-1677. doi:10.1007/s11682-016-9643-1

Van Voorhis, P., Wright, E. M., Salisbury, E. \& Bauman, A. (2010). Women's risk factors and their contributions to existing risk/needs assessment: The current status of a genderresponsive supplement. Criminal Justice and Behavior, 37, 261-288.

doi: $10.1177 / 0093854809357442$

Vergouwe, Y., Steyerberg, E. W., Eijkemans, M. J. C., \& Habbema, J. D. F. (2005). Substantial effective sample sizes were required for external validation studies of predictive logistic regression models. Journal of Clinical Epidemiology, 58, 475-483.

doi:10.1016/j.jclinepi.2004.06.017

Viljoen, J. L., Beneteau, J. L., Gulbransen, E., Brodersen, E., Desmarais, S. L., Nicholls, T. L., \& Cruise, K. R. (2012). Assessment of multiple risk outcomes, strengths, and change with the START:AV: A short-term prospective study with adolescent offenders. The International Journal of Forensic Mental Health, 11, 165-180.

doi:10.1080/14999013.2012.737407 
Vincent, G. M., Guy, L. S., Fusco, S. L., \& Gershenson, B. G. (2012). Field reliability of the SAVRY with juvenile probation officers: Implications for training. Law and Human Behavior, 36(3), 225-236. doi:10.1037/h0093974

Walby, S. (2004). The Cost of Domestic Violence. Retrieved from Devon County Council website: http://www.devon.gov.uk/de/text/cost_of_dv_report_sept04.pdf

Ward, T., Hudson, S. M., \& Marshall, W. L. (1995). Cognitive distortions and affective deficits in sex offenders: A cognitive deconstructionist interpretation. Sexual Abuse: Journal of Research and Treatment, 7, 67-83. doi:10.1007/BF02254874

Webster, C. D., Douglas, K. S., Eaves, D., \& Hart, S. D. (1997). HCR-20: Assessing the risk of violence-Version 2. Burnaby, BC: Simon Fraser University and Forensic Psychiatric Services Commission of British Columbia.

Webster, C. D., Martin, M. L., Brink, J., Nicholls, T. L., \& Desmarais, S. (2009). Manual for the Short-Term Assessment of Risk and Treatability (START) Version 1.1. Port Coquitlam, BC and Hamilton, ON: Forensic Psychiatric Services Commission and St. Joseph’s Healthcare.

Webster, C. D., Martin, M. L., Brink, J., Nicholls, T. L., \& Middleton, C. (2004). Manual for the Short-Term Assessment of Risk and Treatability (START) Version 1.0, Consultation edition. St. Port Coquitlam, BC and Hamilton, ON: Forensic Psychiatric Services Commission and St. Joseph's Healthcare.

Whitaker, D. J., Le, B., \& Niolon, P. H. (2010). Persistence and desistance of the perpetration of physical aggression across relationships: Findings from a national study of adolescents. Journal of Interpersonal Violence, 25, 591-609. doi: $10.1177 / 0886260509334402$ 
White, J. L., Moffitt, T. E., Caspi, A., Bartusch, D. J., Needles, D. J., \& Stouthamer-Loeber, M. (1994). Measuring impulsivity and examining its relationship to delinquency. Journal of Abnormal Psychology, 103, 192-205. doi:10.1037/0021-843X.103.2.192

Williams, K. R., \& Houghton, A. B. (2004). Assessing the risk of domestic violence reoffending: A validation study. Law and Human Behavior, 28, 437-455.

doi:10.1023/B:LAHU.0000039334.59297.f0

Wilson, N. J. (2011). Release-proposal feasibility assessment-revised (RPFA-R) manual and rating booklet: Version 4 (Unpublished user-manual). Wellington, NZ: New Zealand Department of Corrections.

Wilson, C. M., Desmarais, S. L., Nicholls, T. L., Hart, S. D., \& Brink, J. (2013). Predictive validity of dynamic factors: Assessing violence risk in forensic psychiatric inpatients. Law and Human Behavior, 37, 377-388. doi:10.1037/1hb0000025

Woldgabreal, Y., Day, A., \& Ward, T. (2016). Linking positive psychology to offender supervision outcomes: The mediating role of psychological flexibility, general selfefficacy, optimism, and hope. Criminal Justice and Behavior, 43, 697-721. doi:10.1177/0093854815620816

Wooldredge, J., \& Thistlewaite, A. (2002). Reconsidering domestic violence recidivism: Conditioned effects of legal controls by individual and aggregate levels of stake in conformity. Journal of Quantitative Criminology, 18, 45-70. doi:10.1023/A:1013292812895

Wong, S., \& Gordon, A. E. (1999-2003). The Violence Risk Scale. Unpublished manuscript, Department of Psychology, University of Saskatchewan, Saskatoon, Canada. 
World Health Organization. (2017). Intimate partner and sexual violence against women: Fact sheet. Retrieved from World Health Organization website: http:/www.who.int/mediacentre/factsheets/fs239/en/

World Health Organization \& Pan American Health Organization. (2012a). Intimate partner violence: Understanding and addressing violence against women (Report No. WHO/RHR/12.36). Retrieved from http://apps.who.int/iris/bitstream/10665/77432/1/WHO_RHR_12.36_eng.pdf

World Health Organization \& Pan American Health Organization. (2012b). Femicide: Understanding and addressing violence against women (Report No. WHO/RHR/12.38). Retrieved from http://apps.who.int/iris/bitstream/10665/77421/1/WHO_RHR_12.38_eng.pdf?ua=1

Yang, M., Wong, S. C., \& Coid, J. (2010). The efficacy of violence prediction: A meta-analytic comparison of nine risk assessment tools. Psychological Bulletin, 136, 740-67. doi:10.1037/a0020473

Yesberg, J. A., \& Polaschek, D. L. L. (2015). Assessing dynamic risk and protective factors in the community: Examining the validity of the Dynamic Risk Assessment for Offender Re-entry. Psychology, Crime \& Law, 21, 80-90. doi:10.1080/1068316X.2014.935775

Yesberg, J. A., Scanlan, J. M., Hanby, L. J., Serin, R. C., \& Polaschek, D. L. L. (2015). Predicting women's recidivism: Validating a dynamic community-based 'gender-neutral' tool. Probation Journal, 62, 33-48. doi:10.1177/0264550514562851

Yoon, D., Turner, D., Klein, V., Rettenberger, M., Eher, R., \& Briken, P. (2018). Factors predicting desistance from reoffending: A validation study of the SAPROF in sexual 
offenders. International Journal of Offender Therapy and Comparative Criminology, 62, 697-716. doi:10.1177/0306624X16664379

Zhang, T., Hoddenbagh, J., McDonald, S., \& Scrim, K. (2013). An estimation of the economic impact of spousal violence in Canada, 2009 (Report No. rr12-07-e). Retrieved from Department of Justice Canada website: http://www.justice.gc.ca/eng/rp-pr/cj-jp/fvvf/rr12_7/rr12_7.pd 
Appendix A

Dynamic Risk Assessment for Offender Re-Entry (DRAOR)

\begin{tabular}{|c|c|c|c|}
\hline \multicolumn{4}{|c|}{ Stable Risk Indicators } \\
\hline Indicator & \multicolumn{3}{|c|}{ Score (omit if unknown) } \\
\hline Peer Associations & $\begin{array}{c}0 \\
\text { Not a problem }\end{array}$ & $\begin{array}{c}1 \\
\begin{array}{c}\text { Slight/possible } \\
\text { problem }\end{array}\end{array}$ & $\begin{array}{c}2 \\
\text { Definite } \\
\text { problem }\end{array}$ \\
\hline $\begin{array}{l}\text { Attitudes Towards } \\
\text { Authority }\end{array}$ & $\begin{array}{c}0 \\
\text { Not a problem }\end{array}$ & $\begin{array}{c}1 \\
\text { Slight/possible } \\
\text { problem }\end{array}$ & $\begin{array}{c}2 \\
\text { Definite } \\
\text { problem }\end{array}$ \\
\hline Impulse Control & $\begin{array}{c}0 \\
\text { Not a problem }\end{array}$ & $\begin{array}{c}1 \\
\begin{array}{c}\text { Slight/possible } \\
\text { problem }\end{array} \\
\end{array}$ & $\begin{array}{c}2 \\
\text { Definite } \\
\text { problem }\end{array}$ \\
\hline Problem Solving & $\begin{array}{c}0 \\
\text { Not a problem }\end{array}$ & $\begin{array}{c}1 \\
\begin{array}{c}\text { Slight/possible } \\
\text { problem }\end{array} \\
\end{array}$ & $\begin{array}{c}2 \\
\text { Definite } \\
\text { problem }\end{array}$ \\
\hline Sense of Entitlement & $\begin{array}{c}0 \\
\text { Not a problem }\end{array}$ & $\begin{array}{c}1 \\
\begin{array}{c}\text { Slight/possible } \\
\text { problem }\end{array}\end{array}$ & $\begin{array}{c}2 \\
\text { Definite } \\
\text { problem }\end{array}$ \\
\hline $\begin{array}{l}\text { Attachment with } \\
\text { Others }\end{array}$ & $\begin{array}{c}0 \\
\text { Not a problem }\end{array}$ & $\begin{array}{c}1 \\
\begin{array}{c}\text { Slight/possible } \\
\text { problem }\end{array}\end{array}$ & $\begin{array}{c}2 \\
\text { Definite } \\
\text { problem }\end{array}$ \\
\hline \multicolumn{4}{|c|}{ Total Stable Risk Score } \\
\hline
\end{tabular}




\begin{tabular}{|c|c|c|c|}
\hline \multicolumn{4}{|c|}{ Acute Risk Indicators } \\
\hline Indicator & \multicolumn{3}{|c|}{ Score (omit if unknown) } \\
\hline Substance Abuse & $\begin{array}{c}0 \\
\text { Not a problem }\end{array}$ & $\begin{array}{c}1 \\
\begin{array}{c}\text { Slight/possible } \\
\text { problem }\end{array}\end{array}$ & $\begin{array}{c}2 \\
\text { Definite } \\
\text { problem }\end{array}$ \\
\hline Anger/Hostility & $\begin{array}{c}0 \\
\text { Not a problem }\end{array}$ & $\begin{array}{c}1 \\
\begin{array}{c}\text { Slight/possible } \\
\text { problem }\end{array}\end{array}$ & $\begin{array}{c}2 \\
\text { Definite } \\
\text { problem }\end{array}$ \\
\hline $\begin{array}{l}\text { Opportunity/Access to } \\
\text { Victims }\end{array}$ & $\begin{array}{c}0 \\
\text { Not a problem }\end{array}$ & $\begin{array}{c}1 \\
\text { Slight/possible } \\
\text { problem }\end{array}$ & $\begin{array}{c}2 \\
\text { Definite } \\
\text { problem }\end{array}$ \\
\hline Negative Mood & $\begin{array}{c}0 \\
\text { Not a problem }\end{array}$ & $\begin{array}{c}1 \\
\begin{array}{c}\text { Slight/possible } \\
\text { problem }\end{array}\end{array}$ & $\begin{array}{c}2 \\
\text { Definite } \\
\text { problem }\end{array}$ \\
\hline Employment & $\begin{array}{c}0 \\
\text { Not a problem }\end{array}$ & $\begin{array}{c}1 \\
\text { Slight/possible } \\
\text { problem }\end{array}$ & $\begin{array}{c}2 \\
\text { Definite } \\
\text { problem }\end{array}$ \\
\hline $\begin{array}{l}\text { Interpersonal } \\
\text { Relationships }\end{array}$ & $\begin{array}{c}0 \\
\text { Not a problem }\end{array}$ & $\begin{array}{c}1 \\
\text { Slight/possible } \\
\text { problem }\end{array}$ & $\begin{array}{c}2 \\
\text { Definite } \\
\text { problem }\end{array}$ \\
\hline Living Situation & $\begin{array}{c}0 \\
\text { Not a problem }\end{array}$ & $\begin{array}{c}1 \\
\begin{array}{c}\text { Slight/possible } \\
\text { problem }\end{array}\end{array}$ & $\begin{array}{c}2 \\
\text { Definite } \\
\text { problem }\end{array}$ \\
\hline \multicolumn{4}{|c|}{ Total Acute Risk Score } \\
\hline
\end{tabular}




\begin{tabular}{|c|c|c|c|}
\hline \multicolumn{4}{|c|}{ Protective Factors } \\
\hline Indicator & \multicolumn{3}{|c|}{ Score (omit if unknown) } \\
\hline $\begin{array}{l}\text { Responsive to } \\
\text { Advice }\end{array}$ & $\begin{array}{c}0 \\
\text { Not an asset }\end{array}$ & $\begin{array}{c}1 \\
\begin{array}{c}\text { Slight/possible } \\
\text { asset }\end{array} \\
\end{array}$ & $\begin{array}{c}2 \\
\text { Definite asset }\end{array}$ \\
\hline Prosocial Identity & $\begin{array}{c}0 \\
\text { Not an asset }\end{array}$ & $\begin{array}{c}1 \\
\begin{array}{c}\text { Slight/possible } \\
\text { asset }\end{array}\end{array}$ & $\begin{array}{c}2 \\
\text { Definite asset }\end{array}$ \\
\hline High Expectations & $\begin{array}{c}0 \\
\text { Not an asset }\end{array}$ & $\begin{array}{c}1 \\
\text { Slight/possible } \\
\text { asset }\end{array}$ & $\begin{array}{c}2 \\
\text { Definite asset }\end{array}$ \\
\hline Costs/Benefits & $\begin{array}{c}0 \\
\text { Not an asset }\end{array}$ & $\begin{array}{c}1 \\
\begin{array}{c}\text { Slight/possible } \\
\text { asset }\end{array} \\
\end{array}$ & $\begin{array}{c}2 \\
\text { Definite asset }\end{array}$ \\
\hline Social Support & $\begin{array}{c}0 \\
\text { Not an asset }\end{array}$ & $\begin{array}{c}1 \\
\text { Slight/possible } \\
\text { asset }\end{array}$ & $\begin{array}{c}2 \\
\text { Definite asset }\end{array}$ \\
\hline Social Control & $\begin{array}{c}0 \\
\text { Not an asset }\end{array}$ & $\begin{array}{c}1 \\
\text { Slight/possible } \\
\text { asset } \\
\end{array}$ & $\begin{array}{c}2 \\
\text { Definite asset }\end{array}$ \\
\hline \multicolumn{4}{|c|}{ Total Protective Score } \\
\hline
\end{tabular}

\begin{tabular}{|l|c|}
\hline \multicolumn{1}{|c|}{ Risk Level } & DRAOR Cut-off \\
\hline Low & $\leq 2$ \\
\hline Moderate & 3 to 9 \\
\hline Moderate/High & 10 to 22 \\
\hline High & $\geq 23$ \\
\hline
\end{tabular}




\section{Appendix B}

\section{Description of DRAOR Items ${ }^{37}$}

\section{Stable Subscale}

1. Peer Associations: This item refers to the nature and frequency of associations with criminal individuals. Peers can be partners, family members, friends, or acquaintances with whom the client spends free time. Criminal peers may be those who have committed crime in the past, or would likely accept the client if the client were to commit criminal behavior in the present.

2. Attitudes Towards Authority: This item refers to having a hostile, oppositional, antagonistic, or defiant attitude toward those in authority. Attitude refers to beliefs that authority figures (a) do not deserve to have power over them, (b) do not have a legitimate role to play in keeping order or enforcing rules, (c) do not "play fair" specifically when it comes to the client, or (d) do not deserve respect or consideration from the client. Also, (e) a client may describe avoiding their responsibilities or lying to authority figures as a "game" that everyone plays, suggesting that authority figures do not enforce rules for any higher purpose than to manipulate others and "win" power.

3. Impulse Control: This item refers to either a pattern of (a) the client "doing the first thing that comes into their head" without thinking about the consequences, or (b) the client feeling so overwhelmed by impulses that they tend to give in and behave in ways they say they want to avoid.

4. Problem Solving: This item refers to the client's ability to find solutions to their life problems in a way that takes them away from risk situations and criminal behavior. Good problem solving involves (a) a desire to find non-criminal solutions to problems, (b) thinking through options to decide on the best prosocial solution, and (c) taking action to make the prosocial solution a reality, which involves following through to find new solutions if setbacks come up in the process. Poor problem solving involves (a) a preference for criminal solutions to problems, (b) remaining inactive rather than engaging in proactive solutionfinding, or (c) attempting to fix life problems in a way that causes more serious problems (e.g., puts the individual at risk for new crime, or the solution involves criminal activity).

5. Sense of Entitlement: This item refers to an attitude of self-regard and self-centeredness, at the expense of regard for other's rights. Attitude refers to the clients' personal belief that (a) they deserve to get what they want, no matter the expense to others, (b) others will manipulate, coerce, or deceive them, if they don't manipulate, coerce, or deceive others first, (c) it's only fair that those who fight for their own rights will win out over those who are not as strong, and (d) people who lose out had it coming anyway.

6. Attachment with Others: This item refers to a characteristic, ongoing lack of concern for others, resulting in social disconnection or problematic interpersonal attachments. Poor attachment with others may express as (a) general inattention or indifference to the emotions or needs of others, (b) a callous disregard for the ways others may feel hurt or betrayed by

\footnotetext{
${ }^{37}$ Obtained from Serin $(2007,2015,2017)$.
} 
the client's actions, (c) short-term, superficial relationships characterized by opportunistic exploitation, or (d) complete disinterest toward feeling close to or maintaining relationships with others.

\section{Acute Subscale}

1. Substance Abuse: This item refers to use of unauthorized substances, including illegal drugs and substances banned by supervision order, and the misuse of other substances, including prescription drugs and alcohol.

2. Anger/Hostility: This item refers to the presence of either (a) "hot" emotions, such as high irritability, exasperation, fury, or rage (for example), or (b) attitudes that support the degradation of others, harm to others, or dehumanization of others. Both (a) anger and (b) hostility result in the client presenting as antagonistic, either by (a) behaving antagonistically, showing signs of a bad temper (i.e., clenched fists, speaking loudly, angry facial expressions), or (b) verbal expressions that others (specific individuals, or groups of individuals) do not deserve fair, ethical, or kind treatment. Unlike sadness (anxiety, depression), anger is focused outward, such that the client reports feeling upset at others (but, both types of emotions can occur simultaneously).

3. Opportunity/Access to Victims: This item refers to the immediate availability of opportunities for crime. This is especially important to consider if the client has history victimizing a preferred victim (either one individual, such as an ex-partner, or a specified group of individuals, such as a sex offender with preference for child victims).

4. Negative Mood: This item refers to the presence of unpleasant emotions, especially agitation, distress, anxiety, stress, or sadness. Unlike the "hot emotions" (anger), the focus of these negative moods is turned inward, such that the client reports feeling unsettled and upset inside of themselves (but both types of emotions can occur simultaneously).

5. Employment: This item primarily assesses whether or not the client is currently employed. Other considerations surrounding employment have additional relevance. Specifically, (a) employability (i.e., does the client have the necessary skills to join the workforce?), (b) engagement (i.e., is the client currently satisfied with existing employment?), and (c) effort (i.e., is the client motivated to gain or maintain employment?).

6. Interpersonal Relationships: This item refers to current problems in close interpersonal relationships in the client's life (which can include antagonism, victimization, breakdown, disconnection, social pressure to engage in criminal activity, etc.). The primary consideration should focus upon romantic partnerships, but close other relationships (with family, housemates, business partners, etc.) deserve consideration in the absence of a romantic partnership, or when there is severe distress or the breakdown of a prosocial relationship that is important to the client.

7. Living Situation: This item primarily assesses whether or not the client is currently living in stable, long-term housing. Stable housing can be considered on a continuum from lack of any suitable housing, or homelessness (a definite problem), temporary or possibly problematic 
housing situations, such as residence at a halfway house, or "couch surfing" (a possible problem), to safe, suitable, long-term housing (not a problem). However, residing at a halfway house may be rated as definite problem if the client should currently be putting in effort to find other suitable housing, but is not doing so. Alternately, living in a supported residence may be rated as not a problem if the current situation may serve as a stable longterm solution, or the client has already made plans to enter safe, stable housing after leaving the residence.

\section{Protective Subscale}

1. Responsive to Advice: This item refers to the client expressing openness to receive and take guidance for making lifestyle changes that will lead toward a long-term, crime-free lifestyle. This is the "Do I care, do I listen, and do I act?" component of the beliefs that support a process of desisting from crime.

2. Prosocial Identity: This item refers to the client's internal self-image. This is the "Who am I?" component of the beliefs that support a process of desisting from crime. The purpose of this item is to assess whether the client can imagine and articulate a "future self" that feels comfortable, fulfilled, and satisfied in a fully non-criminal lifestyle.

3. (Realistic) High Expectations: This item refers to clients' attitude toward change. Attitude refers to two specific beliefs about change that are particularly relevant. First, do clients have a high sense of hope that they will be able to make the prosocial changes they desire to make in their lives? Second, do they believe that change will take time and require personal effort, such that they need to work at creating personal change and re-gaining the trust of others? Combining these two beliefs, do they have a sense of hope that they have the skills it will take to work to achieve the non-criminal life they want?

4. Costs/Benefits (Supportive of Staying Crime-free): This item refers to clients' attitude toward the personal value of crime, and how this attitude compares or contrasts with their attitude toward the value of extending effort to stay crime-free. For this item, attitude refers to beliefs that bad outcomes will happen if choices are made to commit crime in the future, but good outcomes will happen if effort is extended toward staying crime-free. For example, the client who is characterized by prosocial costs/benefit beliefs can articulate that (a) they will lose something important to them if they ever commit another crime, because the consequences of crime have high drawbacks to them, and (b) they will gain something important to them if they put effort into staying crime-free, even if it is difficult or unfamiliar to them at first.

5. Social Support: This item refers to whether clients have any meaningful relationships with non-criminal individuals, especially individuals who assist the client by offering relational (and/or material) support to the client. This item refers to the nature and frequency of associations with prosocial individuals. These individuals can be partners, family members, friends, or acquaintances with whom the client spends free time. A key feature that identifies a prosocial individual is that they would be unlikely to view the client favorably if the client were to commit new criminal behavior. 
6. Social Control: This item refers to the effects that existing prosocial relationships are having on clients who have supportive, non-criminal individuals in their lives. This item extends beyond the simple existence of prosocial others in the client's life, and refers to the influence these individuals have on the client. 
Appendix C

Structured Dynamic Assessment Case-Management-21 (SDAC-21)

\begin{tabular}{|c|c|c|c|}
\hline \multicolumn{4}{|c|}{ Stable Risk Indicators } \\
\hline Indicator & \multicolumn{3}{|c|}{ Score (omit if unknown) } \\
\hline Gang Association & $\begin{array}{c}0 \\
\text { Not a problem }\end{array}$ & $\begin{array}{c}1 \\
\text { Slight/possible } \\
\text { problem }\end{array}$ & $\begin{array}{c}2 \\
\text { Definite } \\
\text { problem }\end{array}$ \\
\hline $\begin{array}{l}\text { Attitudes Toward } \\
\text { Authority }\end{array}$ & $\begin{array}{c}0 \\
\text { Not a problem }\end{array}$ & $\begin{array}{c}1 \\
\text { Slight/possible } \\
\text { problem }\end{array}$ & $\begin{array}{c}2 \\
\text { Definite } \\
\text { problem }\end{array}$ \\
\hline Impulse Control & $\begin{array}{c}0 \\
\text { Not a problem }\end{array}$ & $\begin{array}{c}1 \\
\begin{array}{c}\text { Slight/possible } \\
\text { problem }\end{array}\end{array}$ & $\begin{array}{c}2 \\
\text { Definite } \\
\text { problem }\end{array}$ \\
\hline Problem Solving & $\begin{array}{c}0 \\
\text { Not a problem }\end{array}$ & $\begin{array}{c}1 \\
\begin{array}{c}\text { Slight/possible } \\
\text { problem }\end{array}\end{array}$ & $\begin{array}{c}2 \\
\text { Definite } \\
\text { problem }\end{array}$ \\
\hline Sense of Entitlement & $\begin{array}{c}0 \\
\text { Not a problem }\end{array}$ & $\begin{array}{c}1 \\
\text { Slight/possible } \\
\text { problem }\end{array}$ & $\begin{array}{c}2 \\
\text { Definite } \\
\text { problem }\end{array}$ \\
\hline $\begin{array}{l}\text { Attachment with } \\
\text { Others }\end{array}$ & $\begin{array}{c}0 \\
\text { Not a problem }\end{array}$ & $\begin{array}{c}1 \\
\text { Slight/possible } \\
\text { problem }\end{array}$ & $\begin{array}{c}2 \\
\text { Definite } \\
\text { problem }\end{array}$ \\
\hline Substance Abuse & $\begin{array}{c}0 \\
\text { Not a problem }\end{array}$ & $\begin{array}{c}1 \\
\begin{array}{c}\text { Slight/possible } \\
\text { problem }\end{array}\end{array}$ & $\begin{array}{c}2 \\
\text { Definite } \\
\text { problem }\end{array}$ \\
\hline \multicolumn{4}{|c|}{ Total Stable Risk Score } \\
\hline
\end{tabular}




\begin{tabular}{|c|c|c|c|}
\hline \multicolumn{4}{|c|}{ Responsivity Risk Indicators } \\
\hline Indicator & \multicolumn{3}{|c|}{ Score (omit if unknown) } \\
\hline Health Problems & $\begin{array}{c}0 \\
\text { Not a problem }\end{array}$ & $\begin{array}{c}1 \\
\begin{array}{c}\text { Slight/possible } \\
\text { problem }\end{array} \\
\end{array}$ & $\begin{array}{c}2 \\
\text { Definite } \\
\text { problem }\end{array}$ \\
\hline Conduct Issues & $\begin{array}{c}0 \\
\text { Not a problem }\end{array}$ & $\begin{array}{c}1 \\
\begin{array}{c}\text { Slight/possible } \\
\text { problem }\end{array}\end{array}$ & $\begin{array}{c}2 \\
\text { Definite } \\
\text { problem }\end{array}$ \\
\hline Negative Mood & $\begin{array}{c}0 \\
\text { Not a problem }\end{array}$ & $\begin{array}{c}1 \\
\begin{array}{c}\text { Slight/possible } \\
\text { problem }\end{array} \\
\end{array}$ & $\begin{array}{c}2 \\
\text { Definite } \\
\text { problem }\end{array}$ \\
\hline $\begin{array}{l}\text { Unresponsive } \\
\text { Rehabilitation }\end{array}$ & $\begin{array}{c}0 \\
\text { Not a problem }\end{array}$ & $\begin{array}{c}1 \\
\begin{array}{c}\text { Slight/possible } \\
\text { problem }\end{array} \\
\end{array}$ & $\begin{array}{c}2 \\
\text { Definite } \\
\text { problem }\end{array}$ \\
\hline Anger/Hostility & $\begin{array}{c}0 \\
\text { Not a problem }\end{array}$ & $\begin{array}{c}1 \\
\begin{array}{c}\text { Slight/possible } \\
\text { problem }\end{array} \\
\end{array}$ & $\begin{array}{c}2 \\
\text { Definite } \\
\text { problem }\end{array}$ \\
\hline $\begin{array}{l}\text { Offence Mirroring } \\
\text { Behaviours }\end{array}$ & $\begin{array}{c}0 \\
\text { Not a problem }\end{array}$ & $\begin{array}{c}1 \\
\begin{array}{c}\text { Slight/possible } \\
\text { problem }\end{array} \\
\end{array}$ & $\begin{array}{c}2 \\
\text { Definite } \\
\text { problem }\end{array}$ \\
\hline Learning Difficulties & $\begin{array}{c}0 \\
\text { Not a problem }\end{array}$ & $\begin{array}{c}1 \\
\begin{array}{c}\text { Slight/possible } \\
\text { problem }\end{array}\end{array}$ & $\begin{array}{c}2 \\
\text { Definite } \\
\text { problem }\end{array}$ \\
\hline \multicolumn{4}{|c|}{ Total Responsivity Risk Score } \\
\hline
\end{tabular}




\begin{tabular}{|c|c|c|c|}
\hline \multicolumn{4}{|c|}{ Protective Factors } \\
\hline Indicator & \multicolumn{3}{|c|}{ Score (omit if unknown) } \\
\hline $\begin{array}{l}\text { Responsive to } \\
\text { Advice }\end{array}$ & $\begin{array}{c}0 \\
\text { Not an asset }\end{array}$ & $\begin{array}{c}1 \\
\begin{array}{c}\text { Slight/possible } \\
\text { asset }\end{array} \\
\end{array}$ & $\begin{array}{c}2 \\
\text { Definite asset }\end{array}$ \\
\hline Prosocial Identity & $\begin{array}{c}0 \\
\text { Not an asset }\end{array}$ & $\begin{array}{c}1 \\
\text { Slight/possible } \\
\text { asset }\end{array}$ & $\begin{array}{c}2 \\
\text { Definite asset }\end{array}$ \\
\hline High Expectations & $\begin{array}{c}0 \\
\text { Not an asset }\end{array}$ & $\begin{array}{c}1 \\
\begin{array}{c}\text { Slight/possible } \\
\text { asset }\end{array} \\
\end{array}$ & $\begin{array}{c}2 \\
\text { Definite asset }\end{array}$ \\
\hline Costs/Benefits & $\begin{array}{c}0 \\
\text { Not an asset }\end{array}$ & $\begin{array}{c}1 \\
\begin{array}{c}\text { Slight/possible } \\
\text { asset }\end{array} \\
\end{array}$ & $\begin{array}{c}2 \\
\text { Definite asset }\end{array}$ \\
\hline Social Support & $\begin{array}{c}0 \\
\text { Not an asset }\end{array}$ & $\begin{array}{c}1 \\
\begin{array}{c}\text { Slight/possible } \\
\text { asset }\end{array} \\
\end{array}$ & $\begin{array}{c}2 \\
\text { Definite asset }\end{array}$ \\
\hline Social Control & $\begin{array}{c}0 \\
\text { Not an asset }\end{array}$ & $\begin{array}{c}1 \\
\begin{array}{c}\text { Slight/possible } \\
\text { asset }\end{array} \\
\end{array}$ & $\begin{array}{c}2 \\
\text { Definite asset }\end{array}$ \\
\hline Employability & $\begin{array}{c}0 \\
\text { Not an asset }\end{array}$ & $\begin{array}{c}1 \\
\begin{array}{c}\text { Slight/possible } \\
\text { asset }\end{array}\end{array}$ & $\begin{array}{c}2 \\
\text { Definite asset }\end{array}$ \\
\hline \multicolumn{4}{|c|}{ Total Protective Score } \\
\hline
\end{tabular}


Appendix D

Iowa Risk Assessment (IRA)

\begin{tabular}{|c|c|}
\hline Item & Score \\
\hline \multicolumn{2}{|l|}{ Age at classification (client's age on the date the IRA is } \\
\hline 28 or older & 0 \\
\hline 27 or younger & 1 \\
\hline \multicolumn{2}{|l|}{ Age at first adult conviction/juvenile adjudication: } \\
\hline 24 or older & -2 \\
\hline 20 to 23 & 0 \\
\hline 19 or younger & 2 \\
\hline \multicolumn{2}{|l|}{ Prior juvenile commitments: } \\
\hline None & 0 \\
\hline One or more & 2 \\
\hline \multicolumn{2}{|l|}{ Prior adult/juvenile probation/parole supervisions: } \\
\hline None & 0 \\
\hline One or more & 2 \\
\hline \multicolumn{2}{|l|}{ Number of prior probation/parole revocations: } \\
\hline None & 0 \\
\hline One & 2 \\
\hline Two or more & 3 \\
\hline \multicolumn{2}{|l|}{ Felony/misdemeanor convictions (do not exceed 3): } \\
\hline None & 0 \\
\hline Burglary or Robbery & 1 \\
\hline $\begin{array}{l}\text { Theft, Forgery, FUFI, Fraudulent Practices, } \\
\text { OMWOC, or any other theft-related offense }\end{array}$ & 1 \\
\hline Assault, Weapons, Public Order offenses & 1 \\
\hline \multicolumn{2}{|l|}{ Misdemeanor conviction history: } \\
\hline None & 0 \\
\hline Two or more & 1 \\
\hline \multicolumn{2}{|l|}{ Sex: } \\
\hline Female & 0 \\
\hline Male & 2 \\
\hline \multicolumn{2}{|l|}{ Alcohol usage problems: } \\
\hline No interference with functioning (no abuse) & 0 \\
\hline Occasional abuse, some disruption of functioning & 1 \\
\hline Frequent abuse, serious disruption, needs treatment & 2 \\
\hline \multicolumn{2}{|l|}{ Drug: } \\
\hline No interference with functioning (no abuse) & 0 \\
\hline Occasional abuse, some disruption of functioning & 1 \\
\hline Frequent abuse, serious disruption, needs treatment & 2 \\
\hline
\end{tabular}




\begin{tabular}{|l|c|}
\hline Number of address changes in last 12 months: & \\
\hline None & -1 \\
\hline One or more & 1 \\
\hline Companions: & 0 \\
\hline Prosocial friends/associations & 1 \\
\hline Friends/associations primarily prosocial & 2 \\
\hline $\begin{array}{l}\text { Friends/associations completely negative (under } \\
\text { supervision or gang affiliations) }\end{array}$ & -2 \\
\hline Employment: & 0 \\
\hline $\begin{array}{l}\text { Satisfactory employment for one year or longer and } \\
\text { salary sufficient to pay for basic needs }\end{array}$ & 2 \\
\hline Secure employment is not applicable & $\begin{array}{l}\text { Unsatisfactory employment, unemployed, or } \\
\text { unemployable }\end{array}$ \\
\hline
\end{tabular}

\begin{tabular}{|l|c|}
\hline IRA Cut-off Scores & $\begin{array}{c}\text { Level of } \\
\text { Administrative }\end{array}$ \\
\hline-5 to 1 & Minimum \\
\hline 2 to 7 & Low Normal \\
\hline 8 to 11 & High Normal \\
\hline 12 to 14 & Intensive \\
\hline $15+$ & \\
\hline
\end{tabular}

